The Texas Medical Center Library

DigitalCommons@TMC

The University of Texas MD Anderson Cancer Center UTHealth Graduate School of

Biomedical Sciences Dissertations and Theses

(Open Access)
The University of Texas MD Anderson Cancer

Center UTHealth Graduate School of

Biomedical Sciences

\title{
$5-2011$
}

\section{The Role of IL-6 in Adenosine-Mediated Pulmonary Fibrosis}

Mesias Pedroza

Follow this and additional works at: https://digitalcommons.library.tmc.edu/utgsbs_dissertations

Part of the Biochemistry Commons

\section{Recommended Citation}

Pedroza, Mesias, "The Role of IL-6 in Adenosine-Mediated Pulmonary Fibrosis" (2011). The University of Texas MD Anderson Cancer Center UTHealth Graduate School of Biomedical Sciences Dissertations and Theses (Open Access). 142.

https://digitalcommons.library.tmc.edu/utgsbs_dissertations/142

This Dissertation (PhD) is brought to you for free and open access by the The University of Texas MD Anderson Cancer Center UTHealth Graduate School of Biomedical Sciences at DigitalCommons@TMC. It has been accepted for inclusion in The University of Texas MD Anderson Cancer Center UTHealth Graduate School of Biomedical Sciences Dissertations and Theses (Open Access) by an authorized administrator of DigitalCommons@TMC. For more information, please contact digitalcommons@library.tmc.edu.

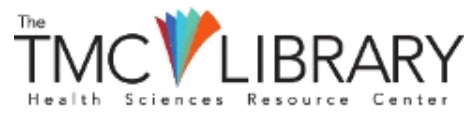




\title{
THE ROLE OF IL-6 IN ADENOSINE- MEDIATED PULMONARY FIBROSIS
}

\author{
By \\ Mesias Pedroza, B.S.
}

APPROVED:

Michael R. Blackburn, Ph.D

Supervisory Professor

Rodney E. Kellems, Ph.D

Sandeep K. Agarwal, M.D./Ph.D

Joseph L. Alcorn, Ph.D

Christopher M. Evans, Ph.D

APPROVED:

George Stancel, Ph.D.

Dean, The University of Texas

Health Science Center at Houston

Graduate School of Biomedical Sciences 


\author{
A \\ DISSERTATION \\ Presented to the Faculty of \\ The University of Texas \\ Health Science Center at Houston \\ and \\ The University of Texas \\ M.D. Anderson Cancer Center \\ Graduate School of Biomedical Sciences \\ in Partial Fulfillment \\ of the Requirements \\ for the Degree of
}

\title{
DOCTOR OF PHILOSOPHY
}

By

Mesias Pedroza, B.S.

Houston, Texas

May, 2011 


\section{ACKNOWLEDGEMENTS}

I would like to thank my advisor, Dr. Michael R. Blackburn, for his guidance and support in my academic studies, for his encouragement and instructions concerning my intellectual development, and for his enthusiasm and values regarding basic research. I am very grateful for his insights, for his time, his valuable skills, for being my mentor, role model, "lab dad," and in a personal way a friend, giving me his understanding, kindness, and flexibility during my own adversities in life. I would like to thank the entire faculty who has served on my committees: Dr. Rodney Kellems, Dr. Sandeep Agarwal, Dr. Joseph Alcorn, Dr. Christopher Evans, Dr. Richard Kulmacz, Dr. Ann-Bin Shyu, and Dr. Hamed Jafar-Nejad. Thanks to Dr. Jianpin Jin for writing my letters of recommendation. Thanks to the Ford Foundation for providing the financial support. Thanks to the members in Dr. Blackburn's lab, especially J. Molina, E. Morschl, Y. Zhou, D. Schneider, T. Le, H. Karmouty, and T. Weng. Special thanks to the administrative staff from the BMB and GSBS, for your valuable work by making mine simpler. Special thanks to Dr. Thomas Goka, my minority mentor. This dissertation is dedicated to my whole family; to my father, Leoncio Pedroza, for your determination in life for us to have a better future; to my mother, Guadalupe Gonzalez, for your patience and devotion in helping us succeed; to my older sister, Avelina Pedroza, for your courage and unconditional support, you took the role of another mother when times were hard; to my older brother, Leo Pedroza, for your kind spirit; and to my younger sister, Carmen Pedroza, for your resilience in life always rising back again. To all of you, thanks for your love and support. 


\section{The Role of IL-6 in Adenosine-Mediated Pulmonary Fibrosis}

Publication No.

Mesias Pedroza, B.S.

Supervisor Professor: Michael R. Blackburn, PhD.

Adenosine is a purinergic signaling molecule that regulates various aspects of inflammation and has been implicated in the pathogenesis of chronic lung diseases. Previous studies have demonstrated that adenosine up-regulates IL-6 production through the engagement of the $A_{2 B}$ adenosine receptor in various cell types, including alveolar macrophages. IL-6 is elevated in mouse models and humans with chronic lung disease, suggesting a potential role in disease progression. Furthermore, chronic elevation of adenosine in the lungs of adenosine deaminase deficient $\left(A a^{-/}\right)$mice leads to the development of pulmonary inflammation, alveolar destruction, and fibrosis, in conjunction with IL6 elevation. Thus, it was hypothesized that IL-6 contributes to pulmonary inflammation and fibrosis in this model. To test this hypothesis, Ada/IL-6 double knockout mice $\left(\mathrm{Ada} / \mathrm{IL}-6^{-/}\right)$were generated to assess the consequences of genetically removing IL-6 on adenosine-dependent pulmonary injury. Ada/IL-6 ${ }^{-1-}$ mice exhibited a significant reduction in inflammation, alveolar destruction, and pulmonary fibrosis. Next, Ada ${ }^{-/}$mice were treated systematically with IL-6 neutralizing antibodies to test the efficacy of blocking IL-6 on chronic lung disease. These treatments were associated with decreased pulmonary inflammation, alveolar destruction, and fibrosis. To determine the role of IL-6 in a second model of pulmonary fibrosis, wild type mice and $I L-6^{-/}$mice were 
subjected to intraperitoneal injections of bleomycin twice a week for four weeks. Results demonstrated that $I L-6^{-/}$mice developed reduced pulmonary fibrosis. To examine a therapeutic approach in this model, wild type mice exposed to bleomycin were treated with IL-6 neutralizing antibodies. Similar results were observed as with $A d a^{-/}$mice, namely diminished pulmonary inflammation and fibrosis. In both models, elevations in IL-6 were associated with increased phosphorylated STAT-3 in the nuclei of numerous cell types in the airways, including type II alveolar epithelial cells (AEC). Genetic removal and neutralization of IL-6 in both models was associated with decreased STAT-3 activation in type II AEC. The mechanism of activation in these cells that lack the membrane bound IL-6R $\alpha$ suggests IL-6 trans-signaling may play a role in regulating fibrosis. Characterization of this mechanism demonstrated that the soluble IL-6R $\alpha(\mathrm{sIL-6R} \alpha)$ is upregulated in both models during chronic conditions. In vitro studies in MLE-12 alveolar epithelial cells confirmed that IL-6, in combination with the sIL-6R $\alpha$, activates STAT-3 and TWIST in association with enhancement of epithelial-to-mesenchymal transition, which can contribute to fibrosis. Similarly, patients with idiopathic pulmonary fibrosis demonstrated a similar pattern of increased IL-6 expression, STAT-3 activation, and sIL-6R $\alpha$ increases. These findings demonstrate that adenosine-dependent elevations in IL-6 contribute to the development and progression of pulmonary inflammation and fibrosis. The implications from these studies are that adenosine and/or IL-6 neutralizing agents represent novel therapeutic targets for the treatment of pulmonary disorders where fibrosis is a detrimental component. 


\section{TABLE OF CONTENTS}

ACKNOWLEDGEMENTS iii

LIST OF ILLUSTRATIONS X X

LIST OF ABBREVIATIONS Xiii

CHAPTER ONE - INTRODUCTION

Chronic Lung Disease $\quad 2$

Pulmonary Fibrosis $\quad 3$

$\begin{array}{ll}\text { Adenosine Metabolism and Signaling } & 8\end{array}$

$\begin{array}{ll}\text { Adenosine and Lung Diseases } & 13\end{array}$

$\begin{array}{ll}\text { Adenosine and IL-6 } & 14\end{array}$

$\begin{array}{ll}\text { IL-6 Signaling } & 16\end{array}$

IL-6 in Lung Diseases $\quad 20$

Dissertation Overview $\quad 22$

CHAPTER TWO - EXPERIMENTAL PROCEDURES 25

Generation and Genotyping of Mouse Lines 26

Generation of Double Knock-out Mice 26

$\begin{array}{ll}\text { ADA Enzyme Therapy } & 27\end{array}$

$\begin{array}{ll}\text { Intraperitoneal Bleomycin Treatment } & 27\end{array}$

IL-6 Neutralization Assay: in ADA-Deficient Mice and Bleomycin Model 28

Histology, Bronchial Alveolar Lavage, and Cellular Differentials 28

$\begin{array}{ll}\text { Immunostaining on Mouse Lung Sections } & 29\end{array}$

Immunofluorescence on Mouse Lung Sections 30

$\begin{array}{ll}\text { Mean Chord Length Determination } & 30\end{array}$ 
$\begin{array}{ll}\text { Protein Assays } & 31\end{array}$

$\begin{array}{ll}\text { Western Blot Analysis } & 31\end{array}$

Measurement of Vascular Permeability 32

PAS Mucus Index $\quad 33$

$\begin{array}{ll}\text { Fibrosis Assessment } & 33\end{array}$

$\begin{array}{ll}\text { Collagen Quantification } & 34\end{array}$

Mouse Oxygen Saturation Measurements $\quad 34$

In vitro Stimulation of Alveolar Epithelial Cell Lines 35

Human lung tissue and BAL samples $\quad 35$

$\begin{array}{ll}\text { Statistics } & 36\end{array}$

CHAPTER THREE - THE ROLE OF IL-6 IN ADENOSINE-MEDIATED PULMONARY INJURY

INTRODUCTION

Adenosine Deaminase-Deficient Model 38

Genetic Removal of IL-6 in ADA-Deficient Mice 39

IL-6 Neutralization in ADA-Deficient Mice $\quad 40$

Specific Questions and Experimental Rationale $\quad 40$

RESULTS

IL-6 Expression and STAT-3 Activation in ADA-Deficient Mice 41

Pulmonary Pathology in Ada/IL-6 Double Knockout Mice 43

Pulmonary Inflammation in Ada/IL-6 Double Knockout Mice 46

Pulmonary Fibrosis in Ada/IL-6 Double Knockout Mice 50

Alveolar Destruction in Ada/IL-6 Double Knockout Mice 50

STAT-3 Activation in Epithelial Cells in ADA-Deficient Mice Following Genetic Removal of IL-6 
Pulmonary Pathology in ADA-Deficient Mice following IL-6 Neutralization

Pulmonary Inflammation in ADA-Deficient Mice following IL-6 Neutralization 55

Pulmonary Fibrosis in ADA-Deficient Mice following IL-6 Neutralization 59

Inflammatory Mediators in ADA-Deficient Mice following IL-6 Neutralization 62

Mediators of Alveolar Destruction in ADA-Deficient Mice following IL-6

Neutralization

62

Vascular Permeability in ADA-Deficient Mice following IL-6 Neutralization 64

Mucus Cell Metaplasia in ADA-Deficient Mice following IL-6 Neutralization 67

STAT-3 Activation in Epithelial Cells in ADA-Deficient Mice following Neutralization of IL-6

DISCUSSION

CHAPTER FOUR - THE ROLE OF IL-6 IN BLEOMICIN-INDUCED PULMONARY FIBROSIS

INTRODUCTION

Intraperitoneal Bleomycin-Induced Pulmonary Fibrosis 80

Genetic Removal of IL-6 in the Bleomycin Model 81

IL-6 Neutralization in the Bleomycin Model 82

Specific Questions and Experimental Rationale 82

RESULTS

Pulmonary Pathology in IL-6 Knockout Mice Treated with Bleomycin 84

Pulmonary Inflammation in IL-6 Knockout Mice Treated with Bleomycin 87

Pulmonary Fibrosis in IL-6 Knockout Mice Exposed to Bleomycin 87

STAT-3 Activation in Airway Epithelial Cells in Mice Exposed to Bleomycin 90

Pulmonary Pathology in Bleomycin-exposed Mice Following IL-6 Neutralization

Pulmonary Inflammation in Bleomycin-exposed Mice Following IL-6 Neutralization 
Pulmonary Fibrosis in Bleomycin-exposed Mice Following IL-6 Neutralization

IL-6-Dependent STAT-3 Activation in Epithelial Cells in

DISCUSSION

CHAPTER FIVE - INVESTIGATION OF MECHANISMS OF IL-6 MEDIATED PULMONARY FIBROSIS

INTRODUCTION

Pulmonary Fibrosis and Epithelial-to-Mesenchymal Transition

IL-6 in Pulmonary Fibrosis

Classical vs IL-6 Trans-signaling

Specific Questions and Experimental Rationale

RESULTS

Elevations of sIL-6R $\alpha$ and Proteases in Models and Patients of Pulmonary Fibrosis

IL-6 Tran-signaling and EMT in Human and Murine Alveolar Epithelial Cells

IL-6 Expression and STAT-3 Activation in Patients with Pulmonary Fibrosis

DISCUSSION

CHAPTER SIX - CONCLUDING REMARKS AND FUTURE DIRECTIONS

Conclusion

Future Directions

Summary

REFERENCES 


\section{LIST OF ILLUSTRATIONS}

Figure 1.1 - Adenosine Metabolism and Signaling

Figure 1.2 - IL-6 Signaling Pathway

Figure 1.3 - STAT-3 Activation by gp130

Figure 3.1- IL-6 expression in the lungs of ADA-deficient mice

Figure 3.2- Increased phosphorylated STAT-3 in the lungs of ADA-deficient mice

Figure 3.3 - Schematic Diagram of Methodology Employed in ADA-deficient Mice

Figure 3.4 - Pulmonary phenotypes following genetic removal of IL-6 in ADA-deficient mice

Figure 3.5 - Pulmonary inflammation following genetic removal of IL-6 in ADA-deficient mice

Figure 3.6 - Pulmonary fibrosis following genetic removal of IL-6 in ADA-deficient mice

Figure 3.7 - Alveolar destruction following genetic removal of IL-6 in ADA-deficient mice

Figure 3.8 - STAT-3 activation following genetic removal of IL-6 in ADA-deficient mice

Figure 3.9 - Pulmonary phenotypes following neutralization of IL-6 in ADA-deficient mice

Figure 3.10 - Anti IL-6 exposure in ADA-deficient mice

Figure 3.11 - Pulmonary inflammation following neutralization of IL-6 in ADA-deficient mice

Figure 3.12 - Pulmonary fibrosis following neutralization of IL-6 in ADA-deficient mice 
Figure 3.12 - IL-6 neutralization in ADA-deficient mice is associated with decreased pulmonary fibrosis

Figure 3.13 - Proinflammatory mediators in mice treated with IL-6 neutralizing antibodies

Figure 3.14 - Alveolar destruction following neutralization of IL-6 in ADA-deficient mice

Figure 3.15 - Alveolar destruction mediators in mice treated with IL-6 neutralizing antibodies

Figure 3.16 - Vascular permeability following neutralization of IL-6 in ADA-deficient mice

Figure 3.17 - Mucus Production following neutralization of IL-6 in ADA-deficient mice

Figure 3.18 - STAT-3 activation following genetic removal of IL-6 in ADA-deficient mice

Figure 4.1- Schematic Diagram of Methodology Employed in the Bleomycin Model

Figure 4.2 - Pulmonary phenotypes following intraperitoneal

bleomycin exposure in IL-6 knock out mice

Figure 4.3 - Pulmonary inflammation following intraperitoneal bleomycin exposure in IL-6 knock out mice

Figure 4.4 - Pulmonary fibrosis following intraperitoneal bleomycin exposure in IL-6 knock out mice

Figure 4.5 - STAT-3 activation following genetic removal of IL-6 in bleomycin treated mice

Figure 4.6 - Pulmonary phenotypes following intraperitoneal bleomycin exposure in mice treated with IL-6 neutralization antibodies 
Figure 4.7 - Pulmonary inflammation following intraperitoneal bleomycin exposure in mice treated with IL-6 neutralization antibodies

Figure 4.8 - Pulmonary fibrosis following intraperitoneal bleomycin exposure in mice treated with IL-6 neutralization antibodies

Figure 4.9 - STAT-3 activation following neutralization of IL-6 in bleomycin treated mice

Figure 5.1 - Epithelial to Mesenchymal Transition (EMT)

Figure 5.2 - Elevation levels of sIL-6R $\alpha$ in ADA-deficient mice and bleomycin model

Figure 5.3 - Morphological changes induce by IL-6 trans-signaling in cell culture studies

Figure 5.4 - Characterization of IL-6 trans-signaling in cell culture studies

Figure 5.5 - Characterization of IL-6 expression and STAT-3 activation in human IPF patients

Figure 5.6 - Characterization of IL-6 trans-signaling in human IPF patients

Figure 6.1 - Working Model 


\section{LIST OF ABBREVIATIONS}

ADA

Ada $^{-/-}$

$\mathrm{Ada}^{+}$

Ada/IL-6 ${ }^{--}$

ADP

AMP

ATP

BALF

cAMP

COPD

ECM

ELISA

EMT

IL

IPF

mIL-6R $\alpha$

PAS

PEG

PBS

Q-PCR

RT-PCR

sIL-6R $\alpha$
Adenosine Deaminase

Adenosine Deaminase Deficient

Adenosine Deaminase Heterozygous

Adenosine Deaminase and Interleukin-6 Deficient

Adenosine Diphosphate

Adenosine Monophosphate

Adenosine Triphosphate

Bronchoalveolar Lavage Fluid

Cyclic Adenosine Monophosphate

Chronic Obstructive Pulmonary Disease

Extracellular Matrix

Enzyme Linked Immunosorbent Assay

Epithelial-to-Mesenchymal Transition

Interleukin

Idiopathic Pulmonary Fibrosis

Membrane-bound IL-6 Receptor $\alpha$

Periodic Acid Schiff

Polyethylene Glycol

Phosphate Buffered Saline

Quantitative Polymerase Chain Reaction

Reverse Transcription - Polymerase Chain Reaction

Soluble IL-6 Receptor $\alpha$ 


\section{CHAPTER ONE INTRODUCTION}




\section{CHRONIC LUNG DISEASES}

Chronic lung disease is a general term describing pulmonary disorders where progressive decline in lung function is caused by persistent structural damage (1-3). Chronic lung diseases are expensive, devastating and deadly, representing the third leading cause of death in America following cancer and cardiovascular diseases (4). The most prominent examples of chronic lung diseases are asthma, chronic obstructive pulmonary disease (COPD), and interstitial lung disease (ILD). Asthma is characterized by infiltration of lymphocytes and eosinophils together with airway remodeling, mucus hypersecretion, basement membrane thickening, angiogenesis, and collagen deposition around the bronchial airways (5). In contrast, COPD is characterized by persistent bronchitis and emphysema due to monocytic inflammation and destruction of alveolar spaces caused by imbalances of proteases and antiproteases that regulate matrix metabolism (6). ILDs, such as idiopathic pulmonary fibrosis (IPF), exhibit varying degrees in inflammation and fibrosis with the prominent features of excessive fibroblast proliferation and extensive matrix deposition in the alveolar spaces (7). Accordingly, these diseases are characterized by a high morbidity and mortality rate (4).

The aforementioned chronic lung diseases are associated with various aspects of lung inflammation, tissue damage and remodeling (8). Despite the diverse responses seen in these disorders, a common feature is the recruitment and activation of multiple effector cells that in turn release various mediators that promote additional inflammation and/or tissue remodeling $(1,8)$. Although 
substantial information is available concerning the biogenesis of the inflammatory and pathological features of chronic lung diseases, the mechanisms that promote the amplification and chronic nature of these disorders have not been adequately investigated (9).

This dissertation will focus on examining mechanisms by which the signaling molecule, adenosine, contributes to the amplification of chronic lung diseases. The significance of this work lies in the fact that chronic lung diseases are largely untreatable. Available therapies target the symptomatic features of these disorders, but fail to manage the extensive airway remodeling seen in these patients (10-12). Thus, identifying signaling pathways responsible for the regulation of progressive pulmonary inflammation and persistent lung damage may provide novel therapeutic approaches for these devastating disorders.

\section{PULMONARY FIBROSIS}

Interstitial lung disease (ILD) is a term used to describe a number of heterogeneous lung disorders sharing common features of variable degrees of pulmonary inflammation and extracellular matrix (ECM) remodeling in the parenchyma and alveolar space $(7,13)$. Pulmonary fibrosis is the hallmark of ILDs and represents the end stage of these lung disorders. There are known etiologies for certain ILDs. Known causes of pulmonary fibrosis include environmental exposure to silica (14), asbestos (15), and coal dust, lifestyle factors such as tobacco use (16), chemotherapeutic drug-induced fibrosis (17), and genetic disorders such as scleroderma (18), sarcoidosis (19), and cystic 
fibrosis (20). However, many ILDs are idiopathic in nature. Idiopathic pulmonary fibrosis (IPF) is the most common of these disorders and is associated with an appalling prognosis, much worse than that of many cancers. IPF is generally lethal within three to five years from the time of diagnosis $(13,21)$. The incidence of IPF is estimated to affect 500,000 individuals in the U.S. and Europe, with a $40 \%$ mortality rate (22), affecting usually middle-aged and older adults with a prevalence $20 \%$ higher in males than females (23). The lack of understanding of the pathogenesis of this condition is reflected in the need for effective therapies and our inability to modify the course of the disease.

Pulmonary fibrosis is commonly characterized by uncontrolled fibroblast proliferation, activation, and differentiation into contractile myofibroblasts, leading to ECM deposition that compromises lung function by distorting the pulmonary architecture $(3,7,13)$. The initiation and progression of fibrosis is thought to arise from a dysregulated epithelial-mesenchymal interaction promoting continued epithelial cell injury and leading to an aberrant wound repair mechanism. Upon cellular injury, alveolar epithelial cells undergo apoptosis while secreting mediators that initiate the wound repair process (24). Inflammatory cells migrate to the site of injury and fibroblasts proliferate from the interstitium to the airways in order to form a provisional matrix (25). Following resolution of the source of injury, the provisional matrix is degraded and new epithelialization occurs $(26,27)$. However, if the source of injury persists, or if amplification pathways are engaged, the wound repair mechanism is deregulated and pulmonary fibrosis follows. 
In addition, infiltrating fibroblasts are transformed into myofibroblasts and collagen synthesis ensues in response to biological mediators. Accumulation, proliferation, and activation of fibroblasts and myofibroblast differentiation represent the active site of injury, which is termed the 'fibroblast foci'. The myofibroblast is considered to be the primary effector cell of pulmonary fibrosis. Nevertheless, the origin of the myofibroblast is not clearly established. Three potential sources have been hypothesized for the observed myofibroblast accumulation and differentiation. These are: i) Proliferation and conversion of resident lung fibroblasts into myofibroblasts (28); ii) Bone-marrow derived circulating fibrocytes, which serve as progenitors for interstitial fibroblast with the potential to differentiate into myofibroblasts (29); and iii) The contribution from a process known as epithelial-mesenchymal transition (EMT), which is the differentiation from a epithelial cell to a mesenchymal, fibroblast-like phenotype resulting in myofibroblast accumulation (30). There are studies indicating that "endothelial-mesenchymal transition" also occurs in lung capillary endothelial cells (31). The different sources of myofibroblasts support the notion that fibrosis occurs as the result of abnormal wound healing responses and persistent epithelial injury independent of inflammation.

The histopathology of IPF is best represented by a heterogeneous patchy distribution of fibrosis (predominantly in the basal and subpleural regions of the lobes), activated myofibroblasts accumulation (in subepithelial fibroblastic foci), and ECM deposition (collagen and fibronectin) (32). New vessels are formed around fibroblast foci and dysregulation in the balance of metalloproteases and 
their inhibitors, tissue inhibitors of metalloproteinases (TIMPs). This disruption in proteases and anti-proteases contributes to the deposition and accumulation of ECM, which ultimately occludes the airways rendering the damage to the alveoli irreversible. Failure to regulate unwarranted fibro-proliferation and matrix deposition is at the core of treatment ineffectiveness. New advancements need to be made in our understanding of the pathogenesis of IPF in order to develop novel and effective therapeutic interventions for this devastating disease. This dissertation seeks to address this gap in knowledge.

The underlying mechanisms of fibrosis remain poorly understood. IPF is a crippling disease where the progressive scarring of the lung tissue reduces gas exchange capacity. The clinical course of IPF is characterized by an increased shortness of breath, a dry and hacking cough, decreased exercise capacity, fatigue and malaise (feeling of being unwell), clubbing (widening of tips of fingers and toes), and ultimately death due to respiratory failure (32). In addition, other medical conditions can arise secondary to IPF. Such potentially life-threatening conditions include blood clotting and infections in the lung, collapsed lungs, lung cancer, and heart and respiratory failure resulting from pulmonary hypertension (32). Epithelial damage, tissue injury, and inflammation are involved in the development of IPF; however, no clinical benefits have been obtained using antiinflammatory therapy. A key cytokine that has been established to promote fibrosis is transforming growth factor (TGF- $\beta$ ) (33). TGF- $\beta$ signaling via the Smad3 pathway is critical for the progression of pulmonary fibrosis (34). In addition, Smad2 activation by TGF- $\beta 1$ induces EMT in human A549 alveolar 
epithelial cells (35). Similar EMT induction by TGF- $\beta$ has been observed in primary alveolar epithelial cells, which suggest this process may be a possible source of myofibroblasts in IPF (36). Different mediators are thought to play an important role in pulmonary fibrosis, such as TNF $\alpha$, CTGF, PDGF, endothelin, IL$1 \beta$, GM-CSF, IL-10, and important to this thesis, IL-6 $(37,38)$. TGF- $\beta$ appears to play a central role in the development of pulmonary fibrosis since it is necessary and sufficient to cause fibrotic lung disease in mice (39). Transgenic overexpression of TGF- $\beta$ in mice can promote the development of the chronic progressive nature of pulmonary fibrosis, allowing for examination of drug effects on TGF- $\beta$ pathways (33). The most widely used animal model of pulmonary fibrosis is the bleomycin model. Bleomycin is a glycosylated linear nonribosomal peptide antibiotic used as an anti-cancer treatment for lymphomas, such as Hodgkin's disease (40). Bleomycin is produced by the bacterium, Streptomyces verticillus, and its mechanism of action is through the induction DNA strand breaks (40). Since the lungs lack the cysteine protease enzyme bleomycin hydrolase, the most serious complication of bleomycin use is pulmonary fibrosis. In animal models, bleomycin induces fibrotic changes similar to human disease (i.e. patchy parenchymal inflammation, epithelial cell injury with reactive hyperplasia, and interstitial and intra-alveolar fibrosis) producing different patterns of fibrotic lesions depending on dose and route of application (40). Single doses of bleomycin via intratracheal (i.t.) administration produce reversible subchronic changes, representing an acute model of lung injury. However, repeated doses of bleomycin lead to long-term tissue damage. Intraperitoneal 
(i.p.) injections of bleomycin in mice produce subpleural scarring, whereas i.t. installations lead to fibrotic changes in a bronchiolocentric accentuated manner (40). The disadvantages of this i.t. bleomycin model include the connection with inflammation, which complicates how to differentiate between pro-inflammatory from pro-fibrotic activities, and the production of reversible fibrosis, which correlates poorly to human disease (40). However, repeated i.p. doses of bleomycin in mice produces changes that resemble the progressive and chronic nature of pulmonary fibrosis observed in humans. This rodent model is an excellent tool to investigate fibrotic disorders in order to discover novel treatments for these deadly diseases. To ensure that our findings in these models are relevant to human disease, it is crucial to validate our findings directly on human tissue to discover novel pathways that are involved in the regulation of these fibroproliferative lung diseases and to achieve effective therapeutic intervention. Hence, this dissertation will focus on addressing the role of adenosine and IL-6 in the development of adenosine-mediated pulmonary fibrosis.

\section{ADENOSINE METABOLISM AND SIGNALING}

Adenosine is a signaling molecule that is generated following cellular injury and is able to promote tissue protection and repair through increasing the oxygen supply/demand ratio, enhancing anti-inflammatory properties, and stimulating angiogenesis (41). Upon cellular stress and damage, ATP is released into the extracellular space through poorly understood mechanisms. 
Extracellular ATP interacts with P2 purinergic receptors to serve as a signaling molecule (Fig 1.1). In addition, ATP can be rapidly dephosphorylated to form extracellular adenosine. This process is mediated by extracellular nucleotidases, namely CD39 and CD73. Extracellularly, adenosine is generated via the apyrase (CD39), which dephosphorylates ATP and ADP to form AMP (42), and the ecto5'-nucleotidase (CD73), which dephosphorylates 5'-AMP to form adenosine (43). Therefore, CD73 plays an important role in the regulation of local adenosine production. Intracellularly, adenosine is generated via the cytosolic 5' nucleotidases (CD73) (44) and through S-adenosylhomocysteine hydrolase (45). Extracellular adenosine can be transported inside the cell by the equilibrative and concentrative nucleoside transporters (ENTs and CNTs, respectively) $(46,47)$. Inside the cell, adenosine can be either catabolized to inosine by the enzyme adenosine deaminase (ADA) or re-phosphorylated into AMP, ADP, and ATP by adenosine kinase (AK) (48). Alternatively, AMP can be phosphorylated back to ATP or deaminated to inosine 5'-monophosphate (48) and ammonia by AMP deaminase. Under normal conditions, the concentration of adenosine is maintained in a steady state by cyto5'NT, AK, and ADA. Hence, at conditions of permanent cellular injury, adenosine concentrations increase by the massive release of ATP from the cell and its subsequent dephosphorylation by CD39 and CD73. At the same time, cellular damage induces a rapid increase in intracellular adenosine levels, which inhibits AK and overwhelms ADA (49). In turn, changes in adenosine concentration regulate an array of physiological 


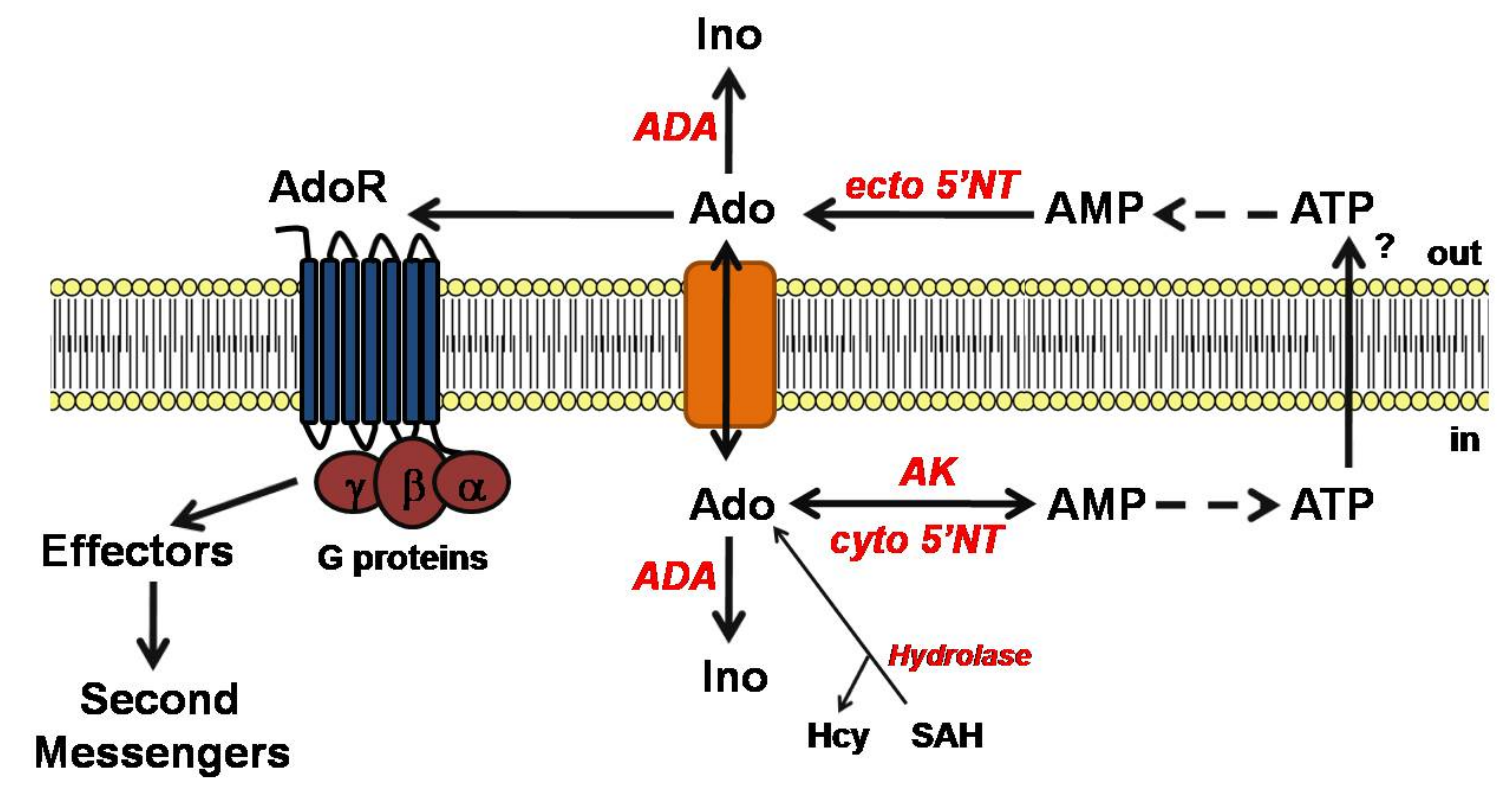

Figure 1.1

Adenosine Metabolism and Signaling. In response to stress, adenosine (Ado) is generated as the result of ATP catabolism. Adenosine deaminase (ADA) deaminates adenosine into inosine (Ino). ATP is dephosphorylated by extracellular nucleotidases (ecto5'NT) such as CD39 and CD73 to form extracellular Ado. Adenosine mediates its effect through four G-protein coupled receptors (AdoR), which are coupled to effector molecules that regulate second messenger systems. Intracellular Ado is produced by dephosphorylating AMP (cyto5'NT) or the hydrolysis of S-adenosylhomocysteine (SAH). Intracellular Ado can be secreted or be phosphorylated back to ATP by adenosine kinase (AK). 
processes by engaging the cell surface adenosine receptors, which lead to modulations in intracellular cAMP and $\mathrm{Ca}^{++}$resulting in either tissue-protective or tissue-destructive effects.

Adenosine levels are elevated in the lungs of humans and mice with chronic lung diseases $(50,51)$. Adenosine signals through the engagement of cell surface G-protein coupled receptors $\left(A_{1} R, A_{2 A} R, A_{2 B} R\right.$, and $\left.A_{3} R\right)$, which are also altered in the lungs of patients with chronic lung disease. Adenosine receptors exert their action by either stimulating ( $\mathrm{G} \alpha \mathrm{S}, \mathrm{A}_{2 \mathrm{~A}} \mathrm{R}$ and $\mathrm{A}_{2 \mathrm{~B}} \mathrm{R}$ ) or inhibiting (Gai, $A_{1} R$ and $A_{3} R$ ) adenylate cyclases and production of the second messenger cAMP (52). Additionally, the $A_{2 B} R$, and $A_{3} R$ are coupled to $G \alpha q$, which activates phospholipases $(53,54)$. Adenosine under normal conditions can elicit tissue protective pathways to promote healing in response to injury. These pathways include the inflammatory response, matrix deposition, and angiogenesis. The activation and effect of these pathways are subject to variation depending on the host genetic composition and the environment. Dysregulation of factors, such as adenosine, can disrupt the pathways that regulate the balance between healing or progression to disease. Excessive adenosine production, such as that found in hypoxic environments of the inflamed lung, can promote mast cell degranulation, induce bronchoconstriction, and exacerbate tissue injury (55-59). Thus, through poorly understood mechanisms, elevations in adenosine lead to diverse actions on inflammation, tissue injury, and tissue remodeling in chronic lung diseases. 
Adenosine deaminase (ADA) is the enzyme responsible for the deamination of adenosine to inosine. Hence, ADA regulates adenosine levels in tissues and in cells. Our lab has genetically engineered mice that lack the gene for ADA ( $\mathrm{Ada}^{-/-}$mice), in which adenosine levels increase progressively. These mice develop pulmonary phenotypes similar to that seen in patients with chronic lung disease, such as alveolar airspace destruction, and pulmonary inflammation and fibrosis (60). Studies in $A d a^{-/-}$mice show that elevations in lung adenosine are sufficient to cause lung inflammation and damage. Furthermore, this pulmonary phenotype can be prevented and reversed by lowering adenosine levels with ADA enzyme replacement therapy (61). This makes $\mathrm{Ada}^{-/-}$mice an excellent model for examining the mechanisms of adenosine driven lung disease. In this model, at the stage of acute tissue injury and low adenosine concentrations, high affinity adenosine receptors $\left(A_{1} R, A_{2 A} R\right)$ are engaged and exert anti-inflammatory and tissue protective pathways $(62,63)$. However, in the setting of prolonged tissue injury and increased levels of adenosine, low affinity adenosine receptors $\left(A_{2 B} R, A_{3} R\right)$ activate pro-inflammatory and tissue destructive pathways $(62,63)$ contributing to the pathology and progressive nature of chronic lung disease. In addition, during the acute phase of lung injury the $A_{2 B} R$ can also provide protective functions despite its low affinity and at low adenosine concentrations (64). Furthermore, mouse models of lung injury, such as transgenic mice overexpressing IL-13 (65) and IL-4 (66), develop pulmonary inflammation, alveolar airspace enlargement, and fibrosis in association with increased adenosine levels. Similarly, the mouse model of bleomycin-induced 
pulmonary fibrosis exhibits increased adenosine concentrations. Consequently, lowering adenosine levels in these models also prevents and reverses features of pulmonary disease $(64,66,67)$. Collectively, these studies suggest that elevations of adenosine regulate pathways that promote the pathogenesis and the chronic pathobiology of lung diseases. However, the detailed mechanisms involved are not known.

\section{ADENOSINE AND LUNG DISEASES}

It has been established that adenosine concentrations and receptor levels are elevated in patients with chronic lung disease (68). Furthermore, adenosine elevation in these patients has been associated with increased inflammation and fibrotic foci development (69). The first link between adenosine and pulmonary disease in humans was made when it was shown that adenosine elicits bronchoconstriction in asthmatic patients but not in normal patients (70). This observation suggested that adenosine signaling is altered in pulmonary diseases. Adenosine therapeutics have been shown to be beneficial as an anti-asthmatic treatments, namely by theophylline-induced inhibition of adenosine receptors (71). Theophylline, a methyl xanthine, inhibits phosphodisterase (PDE) at high concentrations mediating bronchodilatory effects; whereas at low concentrations, theophylline acts as an adenosine receptor antagonist mediating immunoregulatory functions (72). Theophylline, a non-specific adenosine receptor antagonist, provides therapeutic benefit by reducing inflammation via adenosine antagonism. Taken together, these observations imply that 
adenosine has pro-fibrotic or pro-inflammatory effects, depending on the receptor signaling profile and the source of injury. Our lab operates based on the hypothesis made from these observations, namely that adenosine regulates pathways that contribute to the pulmonary pathology seen in chronic lung disease. My thesis focuses on one potential mechanism, which involves the profibrotic mediator IL-6.

\section{ADENOSINE AND IL-6}

Adenosine signaling plays an important role in the regulation of fibrosis, but the mechanisms by which this occurs are not known. Part of the goal of this thesis is to determine the mechanisms by which adenosine regulates features of chronic lung disease. One potential mechanism is the regulation of IL-6 production, a pro-fibrotic cytokine. Adenosine regulates the production of this pleiotropic cytokine in numerous cell types, including airway epithelial cells (73), intestinal epithelial cells (74), pituitary folliculostellate cells (75), astrocytes (76), cardiomyocytes (77), and peritoneal macrophages (78), through the engagement of the $A_{2 B} R$ (79). Treatment of astroglioma cells with a non-specific adenosine agonist is also able to increase IL-6 mRNA and protein levels (80). In addition, adenosine induces IL-6 secretion during active inflammation in the intestinal lumen of mice (81). Also, adenosine is able to stimulate IL-6 production in various cells found in the lung, including bronchial smooth muscle cells (82), alveolar macrophages (83), and lung fibroblasts (28). Thus, there is strong experimental evidence that adenosine regulates IL-6 production. 
IL-6 enhances the innate immune system and protects against tissue damage by inducing an acute phase reaction (84). Besides its functions in inflammation and the acute-phase response, IL-6 plays an important role in haematopoiesis, liver and neuronal regeneration, and B and $\mathrm{T}$ cell growth and differentiation (85). IL-6 is released in response to cytokine induction such as IL1 and TNF- $\alpha(86,87)$. In response to injury and infection, IL-6 is produced by different cells such as, mast cells, eosinophils, endothelial cells, smooth muscle cells, monocytes/macrophages, and B and T cells (88). In addition, IL-6 is able to induce autoimmunity, amplify acute inflammation, and promote chronic inflammation (89). Dysregulation of IL-6 signaling has been implicated in the onset and maintenance of several diseases, such as multiple sclerosis, inflammatory bowel disease, rheumatoid arthritis, and cancer (90). Strong evidence provided by animal models indicates that inhibition of IL-6 represents a promising therapeutic approach for many inflammatory and autoimmune diseases and some cancers (10). The first humanized monoclonal antibody (mAb) targeting IL-6 receptor, Tocilizumab or Actemra, was developed by the Japanese company Chugai (91). This IL-6 inhibitor has been approved and shown to be beneficial for the treatment of Castleman's disease and rheumatoid arthritis. Several anti-IL-6 and anti-IL-6R $\alpha$ mAbs and alternatively native IL-6 blockers (sgp130Fc) are currently under development in different clinical and preclinical studies $(10,91,92)$. However, the effectiveness of such therapies has not been considered for the treatment of chronic lung diseases. 


\section{IL-6 SIGNALING}

IL-6 belongs to the IL-6 family of cytokines, which includes interleukin-11 (IL-11), interleukin-27 (IL-27), ciliary neurotrophic factor (CNTF), cardiotropin-1 (CT-1), cardiotropin-like cytokine (CLC), neuropoietin (NPN), leukemia inhibitory factor (LIF), and oncostatin M (OSM). Each member of this IL-6 cytokine family binds to its own or similar receptor subunit; for instance, IL-6 binds the $80 \mathrm{kDa}$ IL6 binding type I transmembrane glycoprotein termed the membrane bound IL-6 receptor- $\alpha$ (mIL-6R $\alpha, \mathrm{CD} 126)$. In addition, the IL-6 cytokines family share a common receptor component, the type I transmembrane signal transducing unit known as gp130 (CD130) (93). The IL-6R $\alpha$ and gp130 form the IL-6 receptor complex. The mIL-6R $\alpha$ subunit is expressed restrictively in certain cells while gp130 is ubiquitously expressed (94). However, cells that lack the mIL-6R- $\alpha$ can still be activated via a soluble version of the receptor. Thus, the soluble IL-6 receptor $(\mathrm{sIL-6R} \alpha)$ can be bound by ligand and subject a wide variety of cell types functional to IL-6 signaling. This alternative mechanism of IL-6 activating cells lacking the mIL-6R $\alpha$ is termed IL-6 trans-signaling (Fig 1.2). Soluble gp130 acts as an endogenous inhibitor of IL-6 trans-signaling by preventing the activation of target cells since it sequesters the IL- 6 and sIL-6R $\alpha$ complex.

Initially, IL-6 binds with low affinity to the IL6-R $\alpha$ forming a heterodimeric complex with high affinity towards gp130, which consequentially forms a trimolecular complex upon binding. Hence, a hexameric signaling complex is formed by the assembly of two trimolecular complexes consisting of IL-6, IL-6R $\alpha$, and gp130 (95). The gp130 cytoplasmic domain, at tyrosine residue 683 (Y683), 


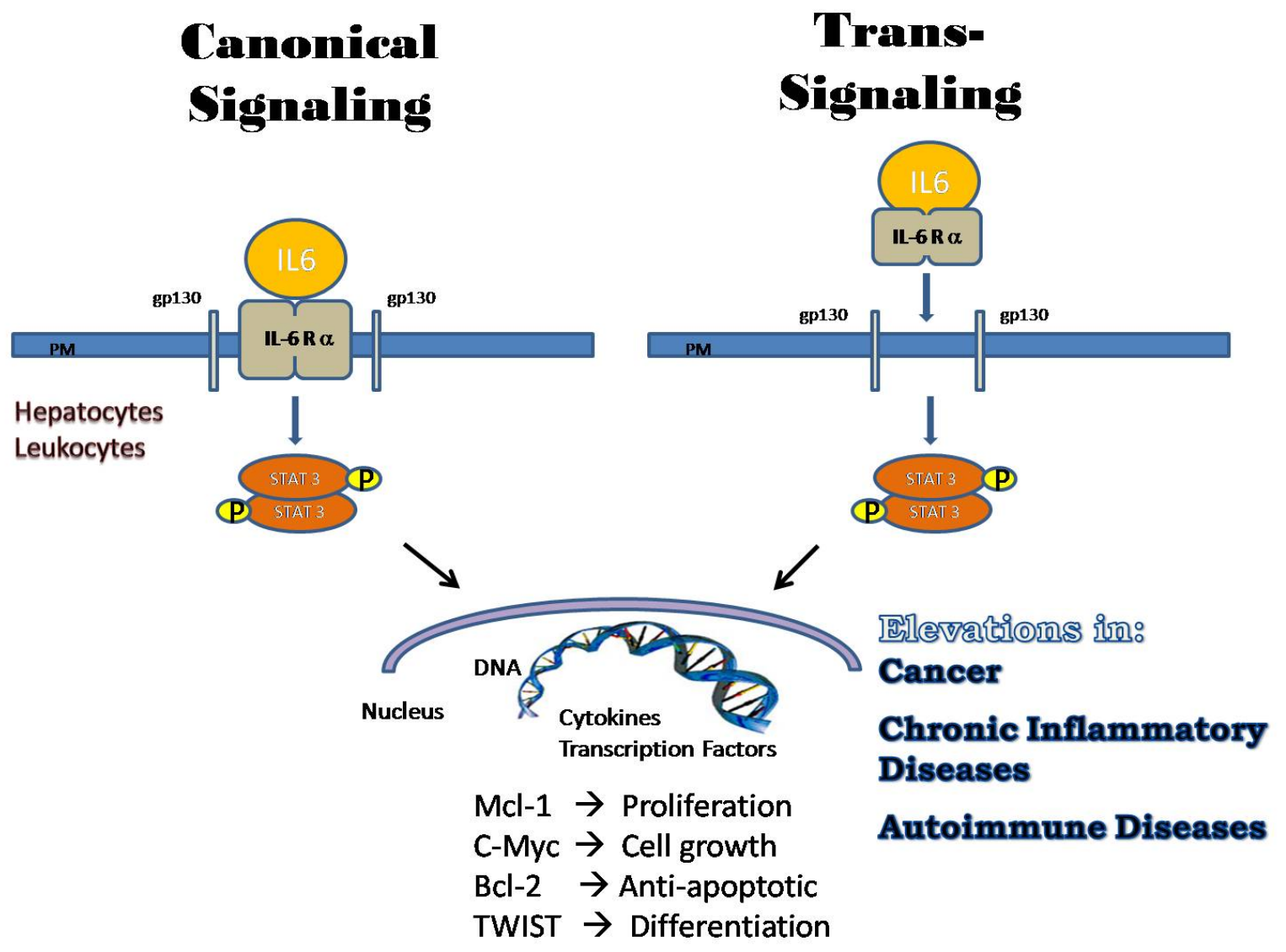

Figure 1.2

IL-6 Signaling Pathway. IL-6 signals through the binding of its receptor, which is composed of two subunits, an alpha subunit for ligand specificity (IL-R $\alpha$ ) and a signal transducing subunit (gp130) that is shared with other cytokines in the IL-6 family. Upon receptor binding, activation of JAK kinases is followed. JAK kinases are responsible for phosphorylating and activating STAT transcription factors, particularly STAT-3. Once STAT-3 is phosphorylated, then it undergoes dimerization and it is translocated into to the nucleus in order to activate transcription of target genes through their STAT-3 response elements. Classical signaling is mediated via the membrane bound IL-6 receptor $\alpha$ (mIL-6R $\alpha$ ). IL-6 trans-signaling refers to the activation of cells that lack the mIL-6Ra. Activation of these cells is mediated via the soluble IL-6 receptor a (sIL-6Ra), which can be produced systemically by alternative splicing or locally by proteolytic cleavage. 
associates with the Janus Kinases (96), namely Jak1, Jak2, and Tyk2. The IL-6 hexameric complex institutes a gp130 conformational change bringing these nonreceptor kinases into close proximity. Upon activation, JAKs transactivate each other by phosphorylating tyrosine residues, which can be recognized by Src homology 2-containing tyrosine phosphotase (SHP-2) and signal transducer and activator of transcription (STATs). In addition, SHP-2 recruitment requires gp130 phosphorylation in order to enhance IL-6 signal transduction (97).

Upon IL-6 activation, the phosphorylation of the tyrosine residues of the gp130 cytoplasmic domain determines the sequential downstream pathways (Fig 1.3). For instance, at residue Y759 the ERK/MAPK cascade is activated primarily for cell survival, whereas at residue Y657, the Akt/PI3K pathway is initiated mainly to enhance proliferation $(97,98)$. On the other hand, gp130 cytoplasmic tyrosine residues, such as Y767, Y814, Y905, and Y915, can activate the STAT-3 pathway in an YXXQ motif dependent manner (97). STATs are characterized by a transient activation with the receptors mediated by their SH2 domains. Upon recruitment of a monomeric STAT to the receptor, phosphorylation ensues, leading the phosphorylated STATs to dimerize. Once the STATs dimerize, they are translocated into the nucleus where they bind the STAT response elements found on the promoter region of target genes $(97,99)$.

Thus, IL- 6 signals by binding to the membrane bound IL-6 receptor (mIL$6 \mathrm{R}-\alpha$ ), which is expressed predominantly on leukocytes and hepatocytes, and then associates with the signal-transducing gp130 protein in order to phosphorylate and dimerize STAT-3 $(84,88)$. Hence, phospho-STAT-3 is an 


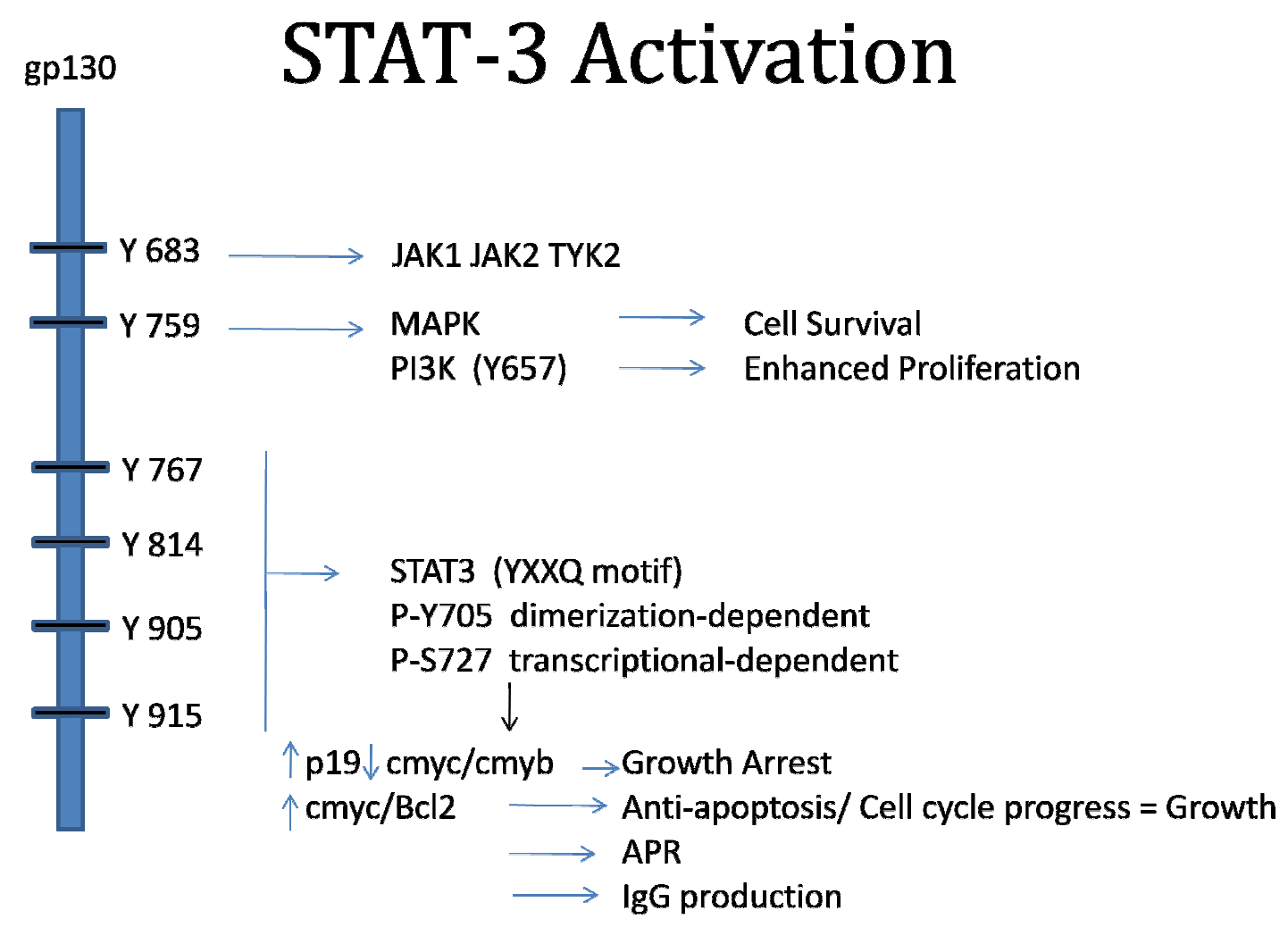

Figure 1.3

STAT-3 Activation by gp130. The signal transducers and activators of transcription (100) family requires JAK-dependent phosphorylation of tyrosine residues in the Src homology 2 domain. JAKs phosphorylate gp130 at tyrosine residue Y683. The gp130 signal transducing unit is able to activate the MAP Kinase (Y759) and the PI3K pathway (Y657). Phosphorylation of gp130 at residues $Y 767, Y 814, Y 904$, and $Y 915$ is able to induce STAT-3 phosphorylation. STAT-3 activation is dependent on the motif YXXQ and tyrosine residues Y705 and S727, which induces dimerization and translocation to the nucleus, respectively. STAT-3 target genes are associated with cell cycle progression, inhibition of apoptosis, and oncogenes, including p19, c-myc and bcl-X. 
indicator of IL-6-induced cellular activation. Phosphorylated STAT-3, once dimerized, is translocated into the nucleus to act as a transcription factor and regulates the expression of target genes $(89,90,101)$.

In addition to this classical pathway, IL-6 binds to the soluble IL-6 receptor (sIL-6R $\alpha$ ) to provide an alternative mechanism of gp130 activation for cells that lack the $\mathrm{mlL}-6 \mathrm{R} \alpha(85,102)$. This trans-signaling mechanism increases the spectrum of IL-6 activities from immune responses to involvement of chronic pathological states (Fig 1.2). This soluble version of the $\mathrm{mIL}-6 \mathrm{R} \alpha$ lacks the transmembrane and cytoplasmic domains, is found at basal levels of $25-35 \mathrm{ng} / \mathrm{ml}$ in BAL from healthy individuals (103), and there are two isoforms generated by posttranscriptional or posttranslational processes (104). Posttranscriptionaly, the slL-6R $\alpha$ can be produced by alternative splicing (10\%) (105) and postranslationaly, by ectodomain shedding (90\%) (106). Alternative spliced sIL6R-mRNA codes for a reading frame shift that generates a novel carboxy terminal protein sequence (GSRRRGSCGL) (107). The ectodomain proteolysis of the mIL-6R $\alpha$ is produced by the $\underline{A} \underline{D}$ isintegrin $\underline{A}$ nd Metalloproteinases (108) gene family members of metalloproteases, such as ADAM10 and ADAM17 (107). ADAM17 is also known as Tumor Necrosis Factor- $\alpha$ Converting Enzyme (TACE), which cleaves membrane bound TNF $\alpha$ thereby activating it (109). The mIL-6R $\alpha$ is targeted by both ADAM10, which is involved in constitutive shedding (110), and by ADAM17, which is involved in stimulated shedding. Under pathophysiological conditions, stimulated shedding is thought to be a master regulator of slL-6R $\alpha$ generation and IL-6 trans-signaling initiation (111). 
ADAM17 cleaves the mIL-6R $\alpha$ close to the transmembrane region, between amino acid position Q357-D358, releasing the slL-6R $\alpha$ into the extracellular space while the short C-terminal remains in the plasma membrane (108). Accordingly, it has been shown that sIL-6R elevation regulates both innate and acquired immunity (98) and dysregulation of this pathway leads to various pathological conditions, since its elevations have been reported in cancer and several chronic inflammatory and autoimmune diseases (112). However, IL-6 trans-signaling has not been investigated in chronic lung diseases. In this dissertation, I will address the role of IL- 6 in pulmonary pathologies and transsignaling in the regulation of fibrosis.

\section{IL-6 IN LUNG DISEASES}

As an inflammatory cytokine, IL-6 is involved in the pathogenesis of lung diseases such as asthma and COPD $(113,114)$, and elevated IL-6 correlates with disease progression and severity. Evidence to support the involvement of IL-6 in chronic lung diseases include the observation that alveolar macrophages isolated from asthmatic patients exposed to allergen demonstrate increased IL-6 secretion (115). In addition, IL-6 expression is observed in mast cells (116) and bronchial epithelial cells (117) from asthmatics, and IL-6 elevations are detected in BALF and sputum from asthmatics (118). In addition, over-expressing IL-6 in lung epithelial cells results in massive airway inflammation (119). In contrast, IL6 is able to diminish airway responsiveness when lung epithelial cells overexpressing IL-6 are challenged with methacholine, which mimics histamine 
effects (120). It is well established that IL-6 is an important cytokine with the ability to act as a pro- or anti-inflammatory or pro-fibrotic agent depending on the environment, and the source and extent of injury. IL-6 is elevated in the lungs of patients suffering from chronic lung diseases; however, its role in these diseases is still being determined.

As a fibrotic cytokine, IL-6 increased expression is associated with fibrotic lung diseases, such as sarcoidosis and IPF (121). Patients with systemic sclerosis and IPF have increased IL-6 levels in their lungs (122). In addition, bleomycin-treated mice exhibit IL-6 elevations during chronic stages of disease (123). Furthermore, IL-6 is able to induce fibrotic effects (122). IL-6-deficient mice exposed to bleomycin exhibit reduced fibrosis compared to control mice (124). IL-6 is able to induce myofibroblast differentiation (28) and collagen synthesis from fibroblasts (125). Also, the severity of pulmonary fibrosis correlates with increased levels of sIL-6R $\alpha$ (125). In addition, fibroblasts isolated from IPF patients treated with IL-6 are apoptosis-resistant and show enhanced proliferation (126). Hence, these studies demonstrate that IL-6 acts as a pro-fibrotic cytokine in pulmonary fibrosis. However, the mechanism by which IL-6 contributes to pulmonary fibrosis remains elusive. This thesis will establish a novel link between adenosine, IL-6, and pulmonary fibrosis.

\section{DISSERTATION OVERVIEW}

In this dissertation I will address the gap in knowledge between the IL-6 signaling pathway and adenosine-mediated pulmonary injury. The pulmonary 
phenotype observed in $\mathrm{Ada}^{-/}$mice demonstrates its utility for examining the mechanisms by which adenosine regulates features of chronic lung disease. Previous studies have demonstrated that elevated levels of adenosine leads to increased production of IL-6 mRNA and protein in the lungs of $A d a^{-/}$mice via engagement of the $A_{2 B} R(79)$. Given the ability of IL-6 to perpetuate the chronic state of various inflammatory diseases, I hypothesize that elevations of IL-6 regulates features of fibrosis in models of adenosine-dependent pulmonary injury. In this thesis, I will present three chapters in which I first examine the role of IL-6 in an adenosine-driven model, namely $A_{d a} a^{-/}$mice, using genetic and pharmacological approaches. To enhance IL-6 relevance in the pulmonary injury and fibrosis observed in $A d a^{-/-}$mice, in the next chapter I will extend these findings using the same approaches (i.e. genetic and pharmacologic) to a more conventional model of pulmonary fibrosis, the bleomycin model. The results from these experiments demonstrate that the genetic removal or neutralization of IL-6 leads to an improvement of pulmonary phenotypes in both models. This suggests that IL-6 is responsible for some key features of pulmonary inflammation and fibrosis. In the process, I determine that phospho-STAT-3 is activated in epithelial cells in the lung, which leads to my description in the last chapter of the potential regulatory mechanisms involve in fibrosis. The IL-6dependent activation of alveolar epithelial cells (AECs) in both models suggests that IL-6 trans-signaling may play a role in activating AECs and regulating fibrosis. One of the key processes for driving fibrosis, namely EMT, can be directly driven by IL-6. Thus, I found evidence that IL-6 trans-signaling was able 
to induce and enhance EMT in vitro. Finally, these observations from animal and cellular models are significant for human disease. I demonstrate that these pathways are elevated in samples collected from patients with COPD and IPF in a similar manner as in our animal models. In this dissertation, these findings demonstrate that adenosine-dependent elevations of IL-6 contribute to the development and progression of pulmonary inflammation and fibrosis. Hence, the IL-6 signaling mechanism represents a potential therapeutic target for these deadly disorders. Thus, this study has enabled the identification of signaling pathways employed in the exacerbation of pulmonary phenotypes in chronic lung diseases. 


\section{CHAPTER TWO EXPERIMENTAL METHODS}




\section{GENERATION AND GENOTYPING MOUSE LINES}

$\mathrm{Ada}^{-/-}$mice were generated and genotyped as described previously (60, 127). Mice homozygous for the null Ada allele were designated ADA-deficient $\left(A d a^{-/}\right)$, while mice heterozygous for the null Ada allele were designated as ADAcompetent mice $\left(\mathrm{Ada}^{+}\right)$. All mice were on a mixed 129/C57BL/6J background, and all phenotypic comparisons were performed among littermates. In addition, IL-6-deficient mice congenic on the C57BL/6J background were obtained from Jackson Laboratory (Bar Harbor, ME, USA). Animal care was in accordance with institutional and NIH guidelines. The University of Texas Health Science Center at the Houston Animal Welfare Committee in Houston, Texas, USA reviewed and approved these studies. Mice were housed in ventilated cages equipped with microisolator lids and maintained under strict containment protocols.

\section{GENERATION OF DOUBLE KNOCK-OUT MICE}

To generate ADA/IL-6 double knockout mice $\left(\right.$ Ada/lL- $\left.6^{-/}\right)$, IL-6-deficient mice congenic on a C57BL/6J background (Jackson Laboratory, Bar Harbor, ME, USA) were backcrossed with $A d a^{-/}$mice also congenic on the C57BL/6J background. Wild type C57BL/6J mice were purchased from Harlan. All phenotypic comparisons were performed among littermates. Animal care was in accordance with institutional and NIH guidelines. These studies were reviewed and approved by the University of Texas Health Science Center at the Houston Animal Welfare Committee in Houston, Texas, USA. 


\section{ADA ENZYME THERAPY}

Polyethylene glycol-modified ADA (PEG-ADA) was generated by the covalent modification of purified bovine ADA with activated polyethylene glycol as

described (64). Ada ${ }^{-/-}$mice were identified at birth by screening for ADA enzymatic activity in the blood as described previously (64). Ada ${ }^{-/-}$mice were maintained on ADA enzyme therapy from postnatal day 1 until postnatal day 25 . Ada $^{-/-}$mice received intra-muscular (i.m.) injections of PEG-ADA on postnatal days $1,5,9,13$, and 17 (0.625 Units, 1.25 Units, 2.5 Units, 2.5 Units, and 2.5 Units, respectively) and intra-peritoneal (i.p.) injections on postnatal days 21 and 25 (5 Units each). Mice were sacrificed on day 27 after the last PEG-ADA injection (postnatal day 43). In addition, ADA/IL-6 double knock out mice were subjected to ADA enzyme therapy until postnatal day 25 and sacrificed on postnatal day 43,27 days after treatment.

\section{INTRAPERITONEAL BLEOMYCIN TREATMENT}

IL-6-competent or IL-6-deficient male mice congenic on C57BL/6J background (Jackson Laboratory, Bar Harbor, ME, USA) 5-weeks old were treated with $0.035 \mathrm{U} / \mathrm{g}$ bleomycin (Teva Parenteral Medicines). PBS was used as vehicle and as control. Bleomycin treatment was administered twice a week for four weeks via intra-peritoneal (i.p.) injections in $100 \mu$ l volume (128). Mice were harvested on day 33 post-treatment. 


\section{IL-6 NEUTRALIZATION ASSAY: IN ADA-DEFICIENT MICE AND BLEOMYCIN MODEL}

Treatments with an IL-6 antibody (30 mg/kg) or control (PBS/isotype antibody/no treatment) were initiated on postnatal day 26. Treatments consisted of subcutaneous injections in $100 \mu$ volume on postnatal day 26,31 , and 37 . Treatment groups included $A_{d a}^{+}$mice or $A d a^{-/}$mice receiving an anti-IL-6 antibody, isotype antibody, PBS (vehicle), or no treatment. All mice were littermates, and both males and females were included in these experiments. In separate experiments, C57BL/6J male mice subjected to intraperitoneal bleomycin administration (twice a week for four weeks, $0.035 \mathrm{U} / \mathrm{g}$ bleomycin in $100 \mu$ l each injection) were treated with an IL-6 antibody $(30 \mathrm{mg} / \mathrm{kg}$ in $100 \mu \mathrm{l}$ volume subcutaneous injections) at day 15, 19, 23, and 27 after first bleomycin injection. Blood was collected using EDTA as an anticoagulant and centrifuged at $1200 \mathrm{rpm}$ for $20 \mathrm{~min}$ to obtain plasma. BAL fluid was collected as described previously and was centrifuged to remove cells. Plasma and the BAL fluid supernatants were analyzed using an ELISA kit to determine exposure levels of the anti-IL-6 antibody.

\section{HISTOLOGY, BRONCHIAL ALVEOLAR LAVAGE AND CELLULAR DIFFERENTIALS}

Mice were anesthetized with $2.5 \%$ avertin. The trachea was cannulated and the lungs were lavaged four times with $0.3 \mathrm{ml}$ of PBS $(0.95-1 \mathrm{ml}$ lavage fluid recovered). Total cell counts were determined using a hemocytometer, and aliquots were cytospun onto microscope slides and stained with Diff-Quick (Dade 
Behring) for cellular differentials. After lavage, the lungs were infused with $10 \%$ buffered formalin at $25 \mathrm{~cm}$ of $\mathrm{H}_{2} \mathrm{O}$ pressure and fixed overnight at $4{ }^{\circ} \mathrm{C}$. Fixed lung samples were dehydrated and embedded in paraffin, and sections $(5 \mu \mathrm{m})$ were collected on microscope slides and stained with H\&E (Shandon-Lipshaw) or Masson's Trichome (EM Science) according to the manufacturer's instructions.

\section{IMMUNOSTAINING ON MOUSE LUNG SECTIONS}

Immunohistochemistry was performed on $5 \mu \mathrm{m}$ sections cut from formalinfixed, paraffin-embedded lungs. Sections were rehydrated through graded ethanols to water, endogenous peroxidases were quenched with $3 \%$ hydrogen peroxide, antigen retrieval was performed (Dako) for $30 \mathrm{~min}$ at $95^{\circ} \mathrm{C}$, and endogenous avidin and biotin was blocked with the Biotin-Blocking System (Dako). Slides were incubated with primary antibodies for mouse IL-6 (1:200 dilution, $1 \mathrm{hr}$ room temperature, Abcam), phospho-STAT-3 (1:100 dilution, $4^{\circ} \mathrm{C}$ overnight, Abcam), or $\alpha$-SMA (1:1000 dilution, $4^{\circ} \mathrm{C}$ overnight, mouse monoclonal, Sigma-Aldrich). All sections were incubated with $A B C$ Elite Streptavidin reagents and appropriate secondary antibodies. Sections were developed with 3,3'diaminobenzidine (Sigma-Aldrich) and counterstained with methyl green or hematoxylin. For $\alpha$-SMA staining, slides were processed with the Mouse on Mouse Kit and the Vector Red Alkaline Phosphatase Substrate Kit (Vector Laboratories). 


\section{IMMUNOFLUORESCENCE ON MOUSE LUNG SECTIONS}

Sections were rehydrated and fixed with acetone and methanol. Endogenous fluorescence was quenched with $1 \% \mathrm{NaBH}_{4}$ for 30 min at room temperature. Slides were incubated with primary antibody to fibronectin (1:400 dilution, $1 \mathrm{hr}$ room temperature, rabbit polyclonal, Sigma-Aldrich) followed by secondary antibody (1:1000 dilution, $1 \mathrm{hr}$ room temperature, donkey anti-rabbit IgG Alexa fluor 555-red, Invitrogen). Sections were covered with Vectashield anti-fade medium with DAPI (VectorLabs).

\section{MEAN CHORD LENGTH DETERMINATION}

The size of alveolar airspaces was determined in pressure-infused lungs by measuring mean chord lengths on H\&E-stained lung sections (79). Representative images were digitized, and a grid consisting of 53 black lines at $10.5 \mu \mathrm{m}$ intervals was overlaid on the image. This line grid was subtracted from the lung images using Image-Pro Plus image analysis software (version 2.0; MediaCybernetics), and the resultant lines were measured and averaged to give

the mean chord length of the alveolar airspaces. The final mean chord lengths represent averages from 10 non-overlapping images of each lung specimen. All quantitative studies were performed blinded with regard to animal genotype.

\section{QUANTITATIVE REAL TIME RT-PCR}

Mice were anesthetized, and the lungs were rapidly removed and frozen in liquid nitrogen. Total RNA was isolated from frozen whole-lung tissue using 
TRIzol reagent (Invitrogen). RNA samples were then DNase treated and subjected to quantitative real-time RT-PCR. The primers, probes, and procedures for real-time RT-PCR were described previously (79). Specific transcript levels for IL-6, CXCL-1, CCL2 (MCP-1), osteopontin (OPN), IL-17, CXCL-2 (MIP-2), TNF- $\alpha$, TIMP-1, MMP-9, MMP-12, CLCA-3, fibronectin, and $\alpha 1-$ procollagen were determined by normalization to $18 \mathrm{~S}$ rRNA and presented as mean normalized transcript levels using the comparative $\mathrm{Ct}$ method $\left(2^{\Delta \Lambda} \mathrm{Ct}\right)$. Primer sequences for the transcripts examined were the same as previously used $(63,64,79)$.

\section{PROTEIN ANALYSIS}

TIMP-1 and sIL-6R $\alpha$ protein levels in BAL fluid were assessed using an ELISA kit (R\&D Systems) according to the manufacturer's instructions.

\section{WESTERN BLOT ANALYSIS}

Frozen lungs were homogenized and lysed on ice with protein lysis buffer (1 $\mathrm{M}$ Tris $(\mathrm{pH} 7.4), 1 \mathrm{M} \mathrm{NaCl}, 1 \%$ Triton $\mathrm{X}-100)$ freshly supplemented with $1 \mathrm{X}$ protease inhibitor mixture (Roche Diagnostics). A $50 \mu \mathrm{g}$ portion of total protein was electrophoresed on $10 \%$ SDS PAGE gels and transferred overnight at $4^{\circ} \mathrm{C}$ to Immobilon-P polyvinylidene difluoride (Millipore), and western blotting was performed. For cell culture, $15 \mu \mathrm{g}$ of total protein was loaded for SDS-PAGE. For primary antibody detection (overnight at $4^{\circ} \mathrm{C}$ ), a rabbit polyclonal anti-mouse was used against phospho-STAT-3 (Abcam 1:500), STAT-3 (Abcam 1:500), $\alpha$ - 
SMA (Sigma 1:500), TWIST (Santa Cruz 1:500) and vimentin (Santa Cruz 1:500), while a mouse monoclonal anti-human was used against e-cadherin (Invitrogen, 1:500), $\alpha$-actin (Sigma 1:5,000), and GAPDH (Invitrogen 1:1,000). Secondary HRP-conjugated antibodies (eBioscience) were applied for 1 hour at room temperature (for P-STAT-3, STAT-3 in 1:1,000; for $\alpha$-SMA, TWIST, vimentin, e-cadherin, and GAPDH at 1:2,000; and for $\alpha$-actin at 1:10,000). Signals were detected by chemiluminesce (Pierce Chemical). Using ImageJ analysis, phospho-STAT-3 band intensity was quantified from four different blots.

\section{MEASUREMENT OF VASCULAR PERMEABILITY}

Lung vascular permeability was quantified from $\mathrm{Ada}^{-/-}$mice and competent mice treated with anti-IL-6 or isotype antibody via subcutaneous injections in 100 $\mu \mathrm{l}$ volume on postnatal day 26,31 , and 37 (as previously performed). At postnatal day 43 , these different treatment groups were subjected to intraperitoneal (i.p.) administration of Evans blue dye (0.2 $\mathrm{ml}$ of $0.5 \%$ in PBS) (129). Four hours later after Evans blue dye administration, lungs were perfused with PBS and harvested. Lung and heart Evans blue concentrations were quantified after formamide extraction $\left(55^{\circ} \mathrm{C}\right.$ overnight) by measuring OD610 with subtraction of reference OD at $450 \mathrm{~nm}$. Evans blue dye contents were determined through comparison to a standard curve generated from dye dilutions. 


\section{PAS MUCUS INDEX}

In separate experiments, $A d a^{-/-}$mice were genotyped and not maintained on ADA enzyme therapy, which develop pulmonary phenotypes by postnatal day 18 and 21, which subsequently die due to pulmonary failure. ADA-competent and $\mathrm{Ada}^{-/}$mice without PEG-ADA treatments were subjected to a single dose (s.c. in $100 \mu \mathrm{l}$ ) of IL-6 antibody (30 mg/kg) or isotype antibody on postnatal day 18. These mice were harvested on postnatal day 21 , their lungs were perfused, fixative with formalin, and paraffin embedded to generate lung sections. Mucus production in bronchial airways was visualized by performing a periodic acidSchiff (PAS)-staining on lung sections and its quantification was determined using Image-Pro Plus analysis software (Media Cybernetics). PAS-positive areas were selected from H\&E lung sections using PhotoshopCS4 in order to determine the average pixel intensity from the digitized images. In order to determine the mucus index, the following equation was employed: mucus index = $(M \times I) / A$, where $M$ stands for the area and I for the intensity of PAS-stained bronchial airways, and A stands for total epithelium. These studies were conducted in a blinded manner, obtaining an average of 10 pictures from each lung to determine the mucus index.

\section{FIBROSIS ASSESSMENT}

To quantify the extent of fibrotic tissue damage, Ashcroft scores were determined on H\&E-stained lung sections from Ada $^{-/}$mice and bleomycin model, with their respective control groups, under both approaches (IL-6 genetic removal 
or neutralization). The Ashcroft scores were determined as previously described (130). These studies were conducted in a blinded manner on 6 mice per group, using $20 \mathrm{X}$ magnification per section.

\section{COLLAGEN QUANTIFICATION}

The Sircol Collagen Assay (Biocolor Assays, Carrick, U.K.) was used to measure soluble collagen in BAL fluid $(100 \mu \mathrm{l})$ according to the manufacturer's instructions.

\section{MOUSE OXYGEN SATURATION MEASUREMENTS}

Physiological assessment measuring arterial oxygen saturation was conducted on conscious mice using the pulse MouseOx software analysis (STARR Life Sciences Corp). Mice treated with bleomycin under the neutralization studies were subjected to the MouseOx. The hair around the neck was removed from mice in order to use the collar clip light sensor. The MouseOx provides real-time percent oxygen saturation of functional arterial hemoglobin by utilizing pulse oximetry measurements of light absorption from the red and infrared LEDs (Light Emitting Diodes). Thus, pulse oximetry measurements of systemic arterial oxygen content are only obtained from pulsating blood, which can only be arterial since non-arterial blood does not change light absorption with heart rate. 


\section{IN VITRO STIMULATION OF ALVEOLAR EPITHELIAL CELL LINES}

Human alveolar epithelial cells, A549, and murine lung epithelial cells, MLE-12 and MLE-15, were obtained as a gift from Dr. Joe Alcorn, University of Texas Health Science Center at Houston. Cells were cultured at $37^{\circ} \mathrm{C}$ with $5 \%$ $\mathrm{CO}_{2}$ in RPIM medium containing $10 \%$ FBS and $1 \%$ antibiotic (A549 and MLE-12) and antimycotic (MLE-15). To test the role of IL-6 trans-signaling on EMT induction, these different cell lines (A549, MLE-12, and MLE-15) were subjected to treatment of: IL-6 alone $(20 \mu \mathrm{g} / \mu \mathrm{l})$, slL-6R $\alpha$ alone $(20 \mu \mathrm{g} / \mu \mathrm{l})$, and the IL-6/sIL$6 \mathrm{R} \alpha$ complex $(40 \mu \mathrm{g} / \mu \mathrm{l}$ total). TGF- $\beta(10 \mu \mathrm{g} / \mu \mathrm{l})$ was used as a positive control in inducing EMT. The media alone was used as a negative control. In addition, the same treatments were performed but with the addition of a phospho-STAT-3 inhibitor $(100 \mu \mathrm{M})(131)$. This inhibitor does not affect STAT-3 phosphorylation, rather inhibits phospho-STAT-3 DNA binding to target promoters. After treatment, these cells were harvested at 24,48 , and 72 hrs post-treatment. After photographing the effects of the different treatment with a inverted bright field microscope, these cells were subjected to western blotting using a RIPA lysis buffer or immunofluorescence using cover slips or chamber slides.

\section{HUMAN LUNG TISSUE AND BAL SAMPLES}

The University of Texas Health Science Center at Houston Committee for the Protection of Human Subjects reviewed and approved the use of human material conducted in this study. All samples collected were already deidentified and exempted, thus providing no ethical concerns. The Lung Tissue Research 
Consortium (LTRC) at the National Institute of Heart Lung and Blood provided us with lung tissue samples from patients of COPD, Stage 0 and Stage 4, and IPF, mild and severe. The LTRC classified the conditions of these patients based on spirometry, pathological examination, and high resolution CT scan. Stage 0 COPD and mild IPF demonstrated preserved lung functions based on the forced expiratory volume in one second (FEV1) and force vital capacity (FVC). Based from these assessments, Stage 0 COPD (FEV1 >80\%) and mild IPF (FVC $>80 \%$ ) were used as control groups compared to Stage 4 COPD (FEV1 $<50 \%$ ) and severe IPF (FVC <50\%). In addition, BAL samples from COPD and IPF patients were obtained through the collaboration with Dr. Murthy, University of Texas Health Science Center at Houston.

\section{STATISTICS}

Values are expressed as mean \pm SEM. As appropriate, groups were compared by analysis of variance; follow-up comparisons between groups were conducted using 2-tailed Student's t test. A P value of $\leq 0.05$ was considered to be significant. 


\section{CHAPTER THREE}

THE ROLE OF IL-6 IN ADENOSINEMEDIATED PULMONARY INJURY 


\section{INTRODUCTION}

\section{ADENOSINE DEAMINASE-DEFICIENT MODEL}

Ada $^{-/-}$mice are a useful model for examining the role of key mediators of lung disease that are driven by increased adenosine levels. Work in $\mathrm{Ada}^{-/}$mice suggests that elevated adenosine is sufficient to cause severe pulmonary pathology, which spontaneously develops as adenosine levels progressively accumulate in the lungs (79). By postnatal day 5, adenosine levels start to increase, and by postnatal day $18 \mathrm{Ada}^{-/-}$mice exhibit activated macrophages, airway epithelial thickening, and increased mucous production (119). Adenosine concentrations found in the lungs of $A d a^{-/}$mice resemble the levels measured in patients of chronic lung disease (50). In addition, $\mathrm{Ada}^{-/-}$mice display a multi organ pathology due to systemic elevations of adenosine and deoxyadenosine, which accumulates in the spleen and thymus leading to a severe combined immunodeficiency (62). Moreover, the pulmonary phenotype in $\mathrm{Ada}^{-/}$mice is characterized by a Th2 cytokine response (62) that is associated with the upregulation of cytokines, chemokines, and mediators of airspace enlargement that promote pulmonary pathology (82). This is similar to what is seen in patients with chronic lung disease. Furthermore, the pathology observed in the lungs of $A d a^{-/}$mice correlates with histopathologic features observed in chronic lung disease (62). These features include eosinophilia, mucus metaplasia, mast cell degranulation (as seen in asthma), neutrophilia, alveolar macrophage activation, distal air-space enlargement (as seen in COPD), and subepithelial and distal airway fibrosis (as seen in IPF) $(64,65)$. Additionally, Ada ${ }^{-/}$mice treated with 
ADA enzyme therapy from birth develop normally and do not exhibit increased levels of adenosine or chronic lung disease. Moreover, ADA enzyme therapy is able to reduce adenosine levels and reverse the features of pulmonary disease with the exemption of airspace enlargement (62). Thus, adenosine signaling is able to directly cause lung pathology in this model making it useful for assessing the role of adenosine in chronic lung disease.

\section{GENETIC REMOVAL OF IL-6 IN ADA-DEFICIENT MICE}

Studies in $A_{d a}{ }^{-/-}$mice demonstrate that IL-6 is elevated in conjunction with increased lung inflammation, mucus production, and fibrosis $(82,122)$. Preliminary data shows that IL-6 production remains unchanged in Ada $^{-/-}$mice until adenosine levels begin to increase (Corrigan, unpublished data). In addition, treatment of $\mathrm{Ada}^{-/-}$mice with a selective $\mathrm{A}_{2 \mathrm{~B}} \mathrm{R}$ antagonist results in reduced pulmonary inflammation and decreased lung damage concurrently with reduced IL-6 production (79). This demonstrates that IL-6 elevations in Ada $^{-/}$ mice are mediated by adenosine in an $\mathrm{A}_{2 \mathrm{~B}} \mathrm{R}$-dependent manner. Furthermore, IL-6 levels have been shown to be elevated in patients with chronic lung disease in correlation with disease severity and morbidity $(113,114)$. Given the ability of IL-6 to perpetuate the chronic state of various inflammatory diseases (88-90), we hypothesized that elevations of IL-6 regulate features of chronic lung diseases in Ada ${ }^{-/-}$mice. To determine the contribution of IL-6, Ada/IL-6 double knockout mice $\left(A d a / I L-\sigma^{/-}\right)$were generated and their pulmonary phenotypes were analyzed 
in comparison with $\mathrm{Ada}^{-/-}$mice. These genetic studies serve to characterize the phenotypic changes in $A d a^{-/-}$mice associated with IL-6.

\section{IL-6 NEUTRALIZATION IN ADA-DEFICIENT MICE}

The pulmonary phenotype observed in $\mathrm{Ada}^{-/-}$mice demonstrate its utility for examining the mechanisms by which adenosine regulates features of chronic lung disease. In this model, adenosine regulates the pleiotropic cytokine IL-6, which has different effects on inflammation and fibrosis. Genetic removal of IL-6 in $\mathrm{Ada}^{-/-}$mice decreases pulmonary pathology. This suggests that IL-6 regulates inflammation and fibrosis in this model. As an additional mechanism to assess the role of IL-6 in the development of pulmonary phenotypes with the emphasis on pursuing therapeutic approaches, $A d a^{-/-}$mice were treated systemically with IL-6 neutralizing antibodies. This neutralization approach demonstrated therapeutic benefits in attenuating chronic pulmonary phenotypes in the $\mathrm{Ada}^{-/-}$ mice. Thus, neutralization of IL-6 in this model revealed that IL-6 signaling is involved in the initiation, maintenance and/or progression of chronic inflammation and fibrosis. The significance of this study is highlighted by the fact that there is a lack of effective therapies that halt the progression of chronic lung diseases (132).

\section{SPECIFIC QUESTIONS AND EXPERIMENTAL RATIONALE}

Extensive studies have demonstrated that IL-6 is induced by adenosine in a variety of cells, such as peritoneal and alveolar macrophages, bronchial 
smooth muscle cells, and human lung fibroblasts (133-135). In Ada ${ }^{-/-}$mice, IL-6 is elevated in conjunction with chronic pulmonary phenotypes. Antagonism of the $A_{2 B} R$ in this model leads to decreased IL-6 levels and diminished features of chronic lung disease. Furthermore, this relationship between adenosine and IL-6 is via the engagement of the $A_{2 B} R(79)$. However, the source of IL-6 production and the cellular targets of IL-6 remain unknown. Identification of the cells that express IL-6 and the cells that are targeted by $\mathrm{IL}-6$ are both critical in understanding the signaling mechanisms by which IL-6 exerts its function in this model. Thus, in this chapter I characterize IL-6 expression and its contribution via phospho-STAT-3 activation in the lung disease seen in $\mathrm{Ada}^{-/-}$mice in order to reveal potential signaling pathways that may be involved in human patients with chronic lung diseases. This study provides a better understanding for the contribution of IL-6 in the lungs of $A d a^{-/-}$mice and the involvement of IL-6 in chronic lung diseases.

\section{RESULTS}

\section{IL-6 EXPRESSION AND STAT-3 ACTIVATION IN ADA-DEFICIENT MICE Elevated expression of IL- 6 in the lungs of $A d a^{-/-}$mice}

Increased levels of IL-6 in the lungs of $A d a^{-/-}$mice have been proposed to be mediated by the $A_{2 B} R$ (79). To confirm IL-6 elevations during active disease in $\mathrm{Ada}^{-/-}$mice, quantitative RT-PCR whole-lung RNA extracts (Fig 3.1a) and IL-6 protein measurements in bronchoalveolar lavage (46) fluid (Fig 3.1b) were 
determined at a stage when adenosine levels and tissue pathology were increased (119). Results demonstrated a 2.5 fold increase of IL-6 transcripts and a 1.7 fold increase of IL-6 protein in the BAL fluid of $A d a^{-/-}$mice. IL-6 levels in the lungs of $A d a^{-/}$mice were diminished following treatment with PEG-ADA to lower adenosine levels (Fig 3.1b). To explore the source of IL-6 in this model, immunolocalization was performed on lung sections. Alveolar macrophages and bronchial airway epithelial cells were identified as the major cells producing IL-6 in the lungs of $\mathrm{Ada}^{-/-}$mice (Fig 3.1c). Interestingly, IL-6 immunoreactivity on these cells was reduced following PEG-ADA treatment. These findings demonstrate that alveolar macrophages and bronchial airway epithelial cells produce IL-6 in an environment of elevated adenosine.

\section{Increased phospho-STAT-3 in the lungs of ADA-deficient mice.}

IL-6 binds to the IL-6 receptor to ultimately lead to the phosphorylation of STAT-3 and its translocation to the nucleus of cells in order to activate the transcription of target genes (90). Thus, the use of phosphorylated antibodies against STAT-3 serves to indicate cellular targets of IL-6. To examine IL-6 cellular target activation, STAT-3 western blot analysis was performed on whole lung homogenates. This analysis demonstrated increased phospho-STAT-3 activation in Ada $^{-/-}$mice compared to ADA-competent mice (Fig 3.2a). PhosphoSTAT-3 levels were significantly diminished in the lungs of $\mathrm{Ada}^{-/-}$mice treated with PEG-ADA (Fig 3.2b). To identify the potential IL-6 cellular targets in this model, immunohistochemical experiments were performed on lung sections to 
determine phospho-STAT-3 localization. In $\mathrm{Ada}^{-/-}$mice, nuclear phospho-STAT3 staining was observed on bronchial airway epithelial cells and predominantly on airway epithelial cells in the alveolar airspaces (Fig 3.2c). The increased phosho-STAT-3 activation in these cells was reversed by treatment with PEGADA (Fig 3.2c, upper and lower right panel). These results suggest that phospho-STAT-3 activation on bronchial epithelial cells may be an indication of increased IL-6 signaling in these cells.

\section{Ada/IL-6 DOUBLE-KNOCKOUT MICE EXHIBIT DECREASED PULMONARY INFLAMMATION, FIBROSIS AND STAT-3 ACTIVATION.}

For the genetic studies, ADA/IL-6 double knockout $\left(\right.$ Ada/lL- $\left.6^{-}\right)$mice were generated by breeding $A d a^{-/-}$mice with $I L-\sigma^{-\alpha}$ mice. Ada/IL- $\sigma^{-/}$mice were treated with PEG-ADA since birth to avoid ADA deficiency lethality and to allow for normal development. Enzyme therapy was halted at postnatal day 25 and pulmonary endpoints were analyzed at postnatal day 43 to compare Ada/IL-6 ${ }^{/-}$ mice and $\mathrm{Ada}^{-/}$mice and determine the contribution of IL-6 in this model (Fig 3.3).

\section{PULMONARY PATHOLOGY IN Ada/IL-6 DOUBLE KNOCKOUT MICE}

In association with elevations in IL-6, the adenosine-mediated pulmonary injury in the $A d a^{-/-}$mice is characterized by an increased influx of inflammatory cells and fibrosis in the distal airways. Ada/IL- $6^{--}$mice were generated to 


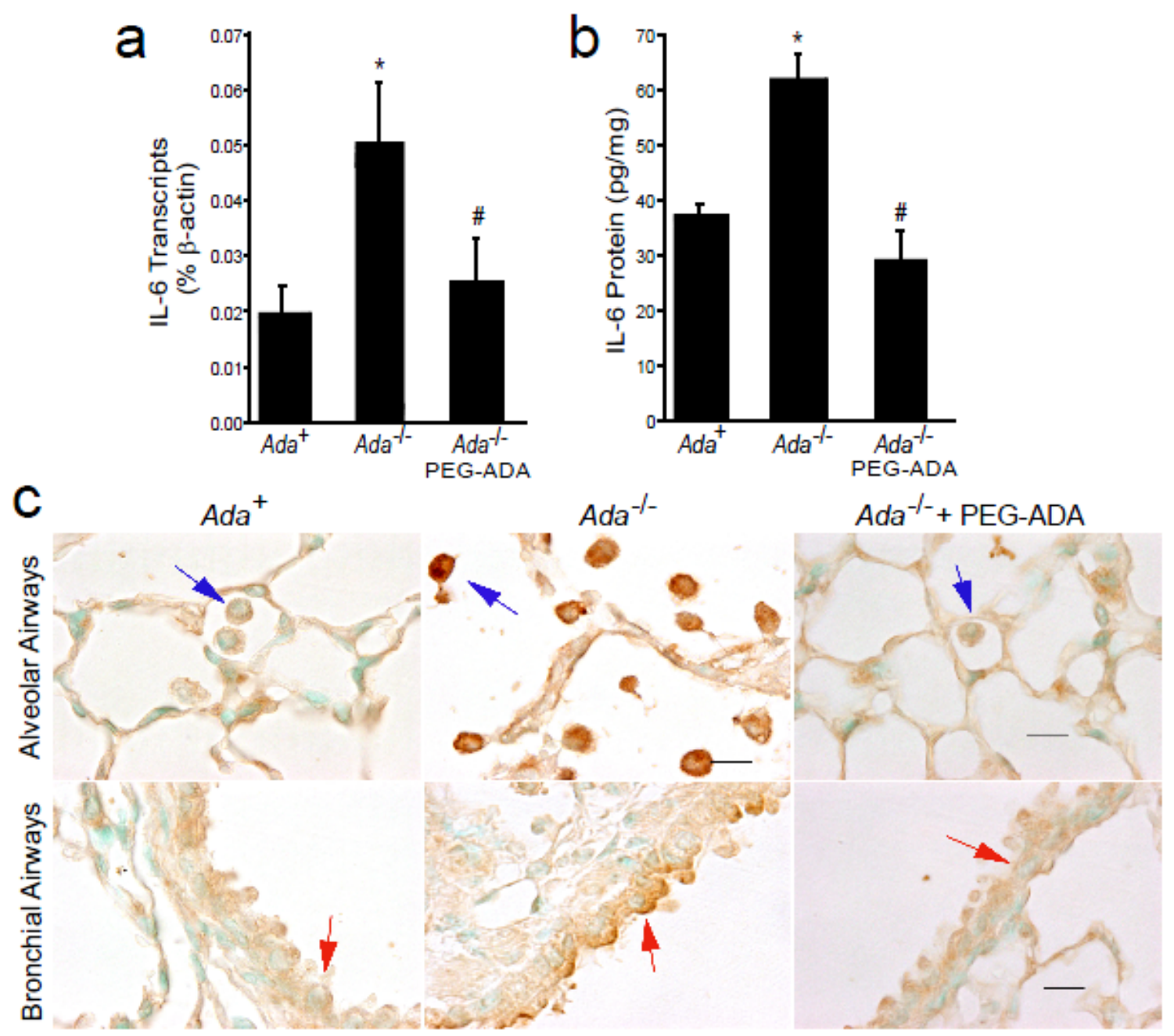

\section{Figure 3.1}

\section{IL-6 expression in the lungs of ADA-deficient mice.}

IL-6 localization, protein expression, and transcript levels were assessed in lung sections, BAL fluid, and whole lungs from postnatal day 18-20 $\mathrm{Ada}^{+}, \mathrm{Ada}^{-/-}$mice, and postnatal day $21 \mathrm{Ada}^{-/-}$mice 72 hours after treatment with PEG-ADA.

(a) IL-6 transcript levels were measured from whole-lung RNA extracts using quantitative RT-PCR with specific IL-6 primers. Data presented as the percentage of $\beta$-actin \pm SEM, $n \geq 4$. (b) IL-6 protein levels were quantified from the BAL fluid using an ELISA kit. Values are presented as $\mathrm{pg} / \mathrm{mg}$ of protein \pm SEM, n $\geq 4 .\left(^{*}, \mathrm{p} \leq 0.001 \mathrm{Ada}^{+}\right.$vs. Ada ${ }^{-/}$and \#, p $\leq 0.01 \mathrm{Ada}^{-/-}$vs. Ada ${ }^{-/-}$PEGADA). (c) Immunohistochemical localization of IL-6 expression, alveolar macrophages (black arrows), bronchial epithelial cells (red arrows). Images are representative of 4 animals from each group. Scale bars: $10 \mu \mathrm{m}$. 

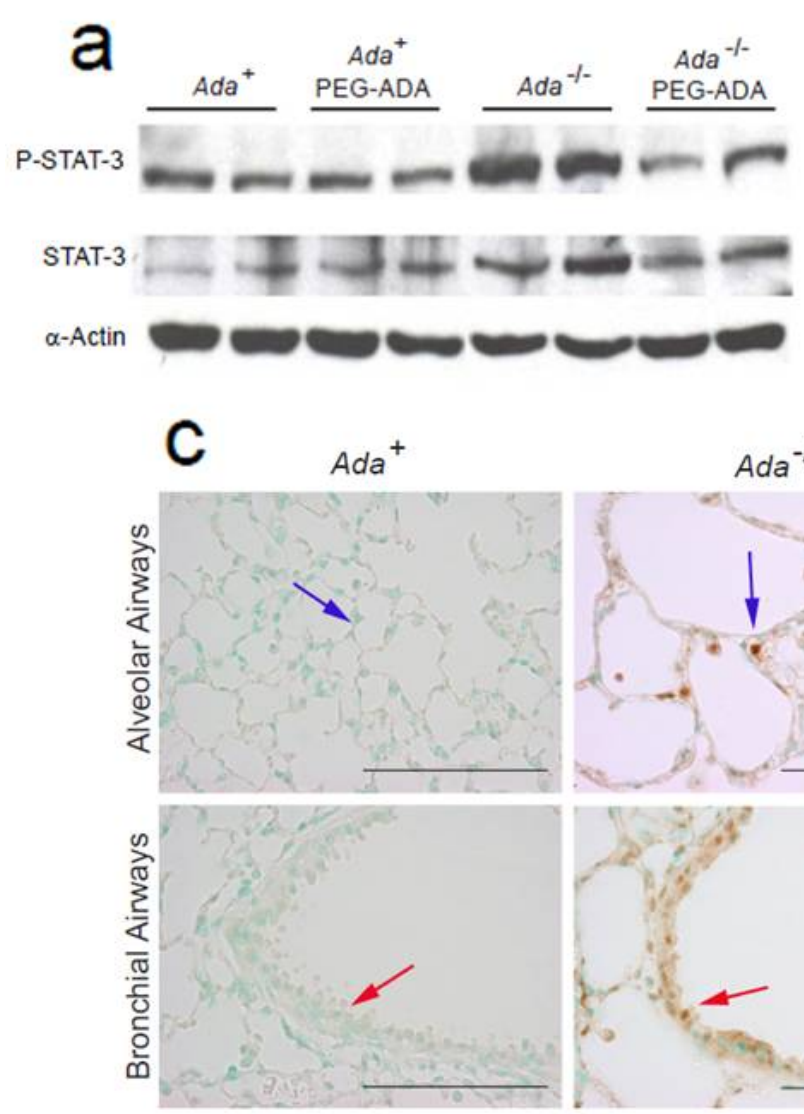

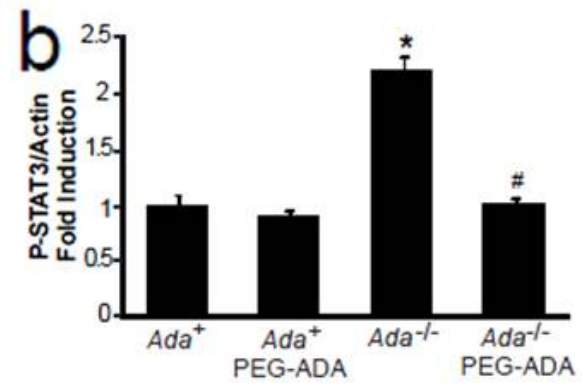

$\mathrm{Ada}^{-/-}+\mathrm{PEG} A D A$
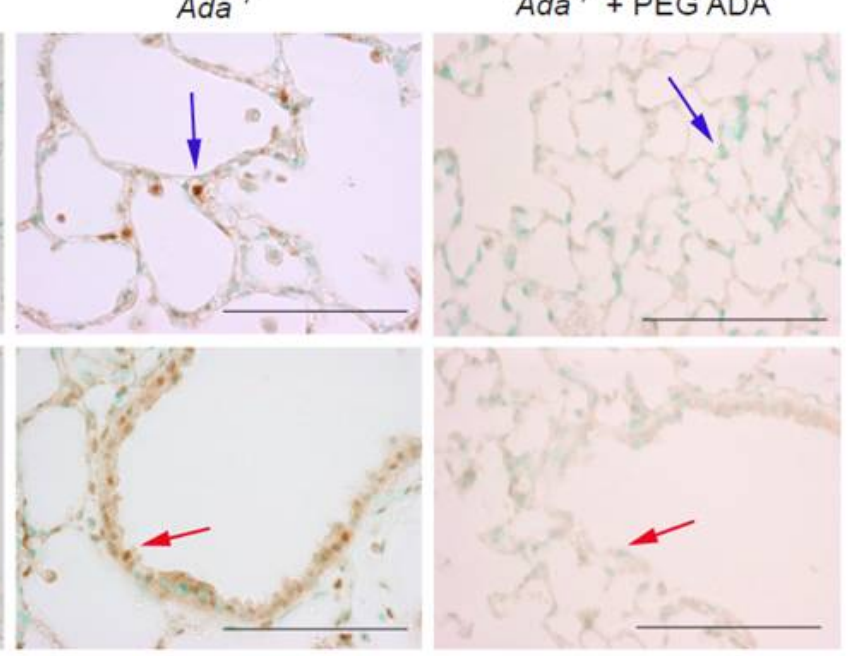

Figure 3.2

\section{Increased phosphorylated STAT-3 in the lungs of ADA-deficient mice.}

Phospho-STAT-3 localization and protein expression were assessed in lung sections from postnatal day $18 \mathrm{Ada}^{+}, \mathrm{Ada}^{-/-}$mice, and postnatal day $21 \mathrm{Ada}^{-/-}$ mice 72 hours after treatment with PEG-ADA. (a) Immunohistochemical localization of phospho-STAT-3 expression, alveolar epithelial cells (blue arrows), bronchial epithelial cells (red arrows). Images are representative of 4 animals from each group. Scale bars: $100 \mu \mathrm{m}$. (b) Phospho-STAT-3 protein expression was measured from whole-lung extracts using western blot analysis with an antibody against phospho-STAT-3. Total STAT-3 levels were also examined and $\alpha$-actin levels were used as loading controls. (c) Phospho-STAT3 band intensity was quantified using ImageJ analysis. Values are presented as the percentage of $\alpha$-actin \pm SEM, $n \geq 4 .\left({ }^{*}, p \leq 0.05\right.$ Ada $^{+}$vs. Ada ${ }^{-/}$and $\#, p \leq 0.05$ Ada $^{-/-}$vs. Ada $^{-/-}+$PEG-ADA). 
determine the contribution of IL-6 to the pulmonary phenotypes observed in $A d a^{-/-}$ mice. Lung histopathology through examination of H\&E sections from these mice demonstrated a reduction in pulmonary injury in Ada/IL- $6^{/-}$mice compared in parallel to $A d a^{-/-}$mice (Fig 3.4). Ada/IL- $6^{-/}$mice were characterized with decreased pulmonary inflammation, reduced airspace enlargement, and diminished fibrosis (Fig 3.4, bottom right panel). This suggests that in the Ada $^{-/-}$ mice, in the setting of increased adenosine concentrations IL-6 contributes to the development of pulmonary pathology including pulmonary inflammation, airway destruction, and fibrosis.

\section{PULMONARY INFLAMMATION IN Ada/IL-6 DOUBLE KNOCKOUT MICE}

Ada/IL- $6^{--}$mice exhibited decreased pulmonary inflammation compared Ada $^{-/-}$mice. BAL was performed on these mice and recovered inflammatory cells were counted using a hemocytometer. Analysis of cellular differentials allowed identification of macrophages, neutrophils, and lymphocytes. Quantification on the level of inflammation based from BAL analysis demonstrated a significant reduction in $A d a / I L-6^{--}$mice of inflammatory cells, including macrophages, neutrophils, and lymphocytes (Fig 3.5a-c) compared to Ada ${ }^{-/-}$mice. These results demonstrate that IL-6 is involved in the regulation of the inflammatory response observed in this model. 


\section{Genetic Removal and Neutralization of IL-6 in ADA-Deficient Mice}
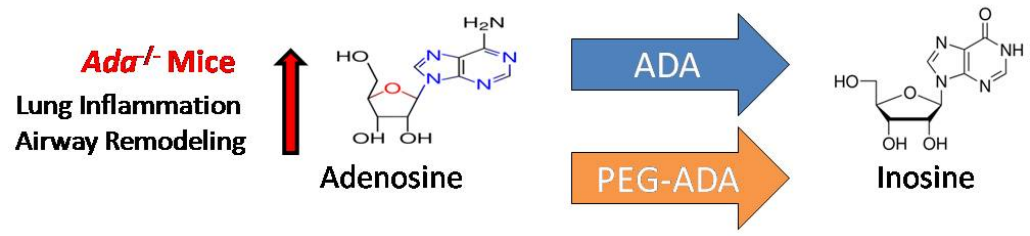

\section{- Genetic Studies}

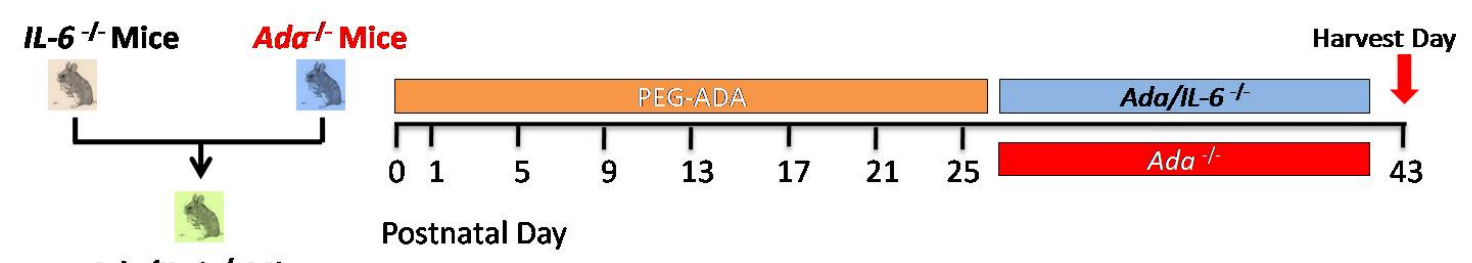

Ada/lL-6 ${ }^{-1-}$ Mice

\section{- Neutralization Studies}

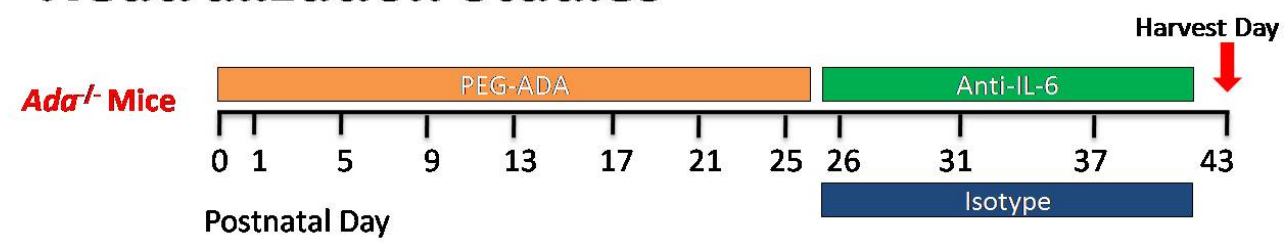

\section{Figure 3.3}

\section{Schematic Diagram of Methodology Employed in ADA-deficient Mice.}

To determine the role of IL-6 in $A d a^{-/-}$mice two approaches were utilized. The genetic studies consisted of generating ADA/IL-6 double knockout ( $\mathrm{Ada} / \mathrm{IL}-\sigma^{/-}$) mice by backcrossing $A d a^{-/-}$mice and $I L-\sigma^{/-}$mice. To avoid ADA deficiency lethality, Ada/IL- $6^{-/}$mice were treated with PEG-ADA since birth until postnatal day 25 to allow for normal development. Once ADA enzyme therapy was halted, pulmonary endpoints were analyzed at postnatal day 43 between $\mathrm{Ada} / \mathrm{IL}-6^{-/}$mice and $\mathrm{Ada}^{-/-}$mice. The neutralization studies consisted of analyzing the therapeutic benefits of neutralizing IL- 6 during the development of disease progression. In this approach, Ada $^{-/-}$mice were treated with PEG-ADA from birth until postnatal day 25 for normal development, followed by treatments of an IL-6 antibody or an isotype control antibody. At postnatal day 43, pulmonary endpoints were analyzed to characterize the effectiveness of neutralizing IL-6. 

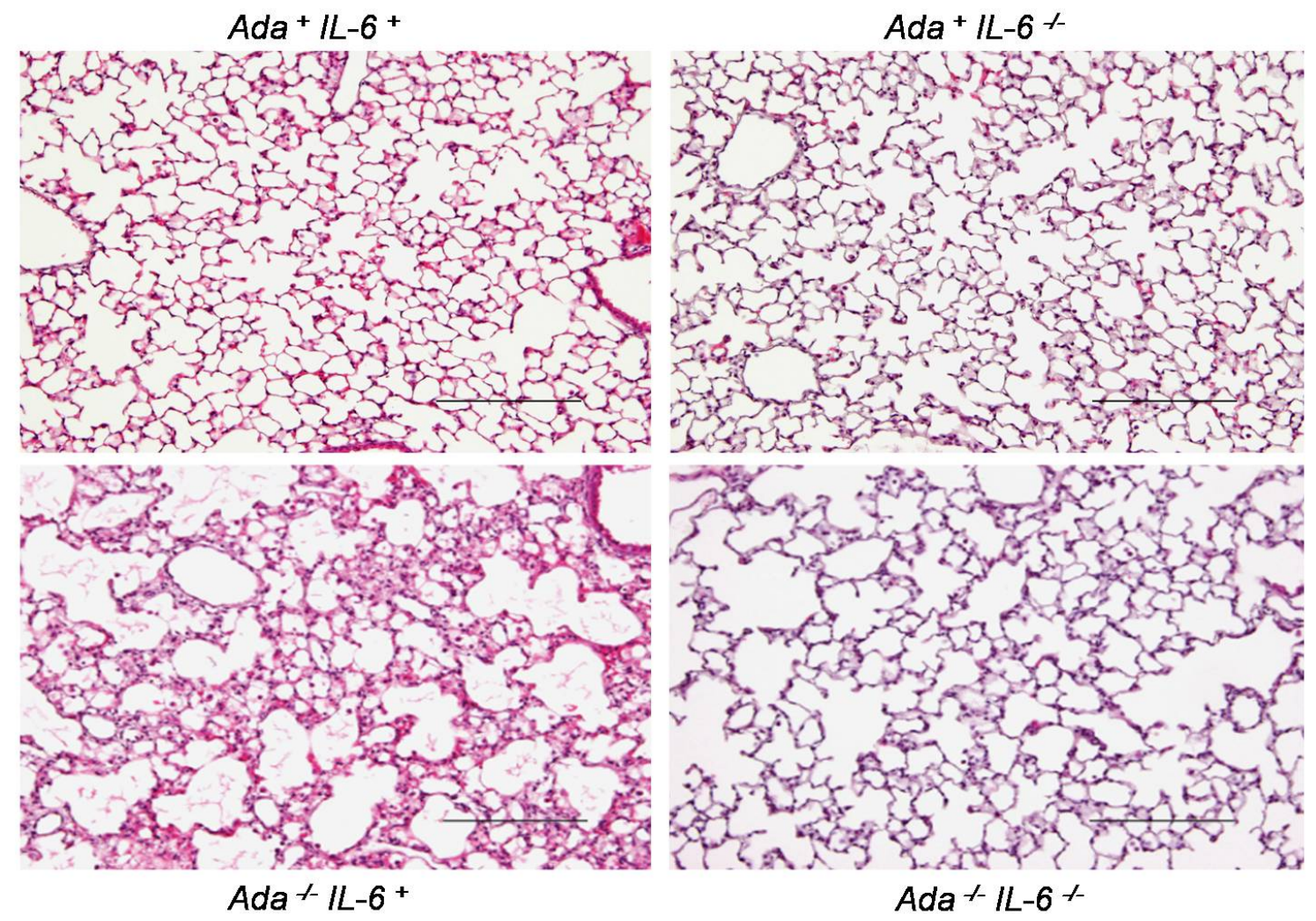

Figure 3.4

Pulmonary phenotypes following genetic removal of IL- 6 in ADA-deficient mice.

Histology of the lungs from postnatal day $43 \mathrm{Ada}^{+}, \mathrm{Ada}^{-/-}$, and $\mathrm{Ada} / \mathrm{IL}-6^{-/}$mice. Examination of lung histology through $\mathrm{H} \& \mathrm{E}$ staining revealed that $\mathrm{Ada} / \mathrm{IL}-6^{-/-}$mice displayed a reduction in lung inflammation, airway enlargement, and fibrosis. Images are representative of 8 animals from each group. Scale bars: $200 \mu \mathrm{m}$. 

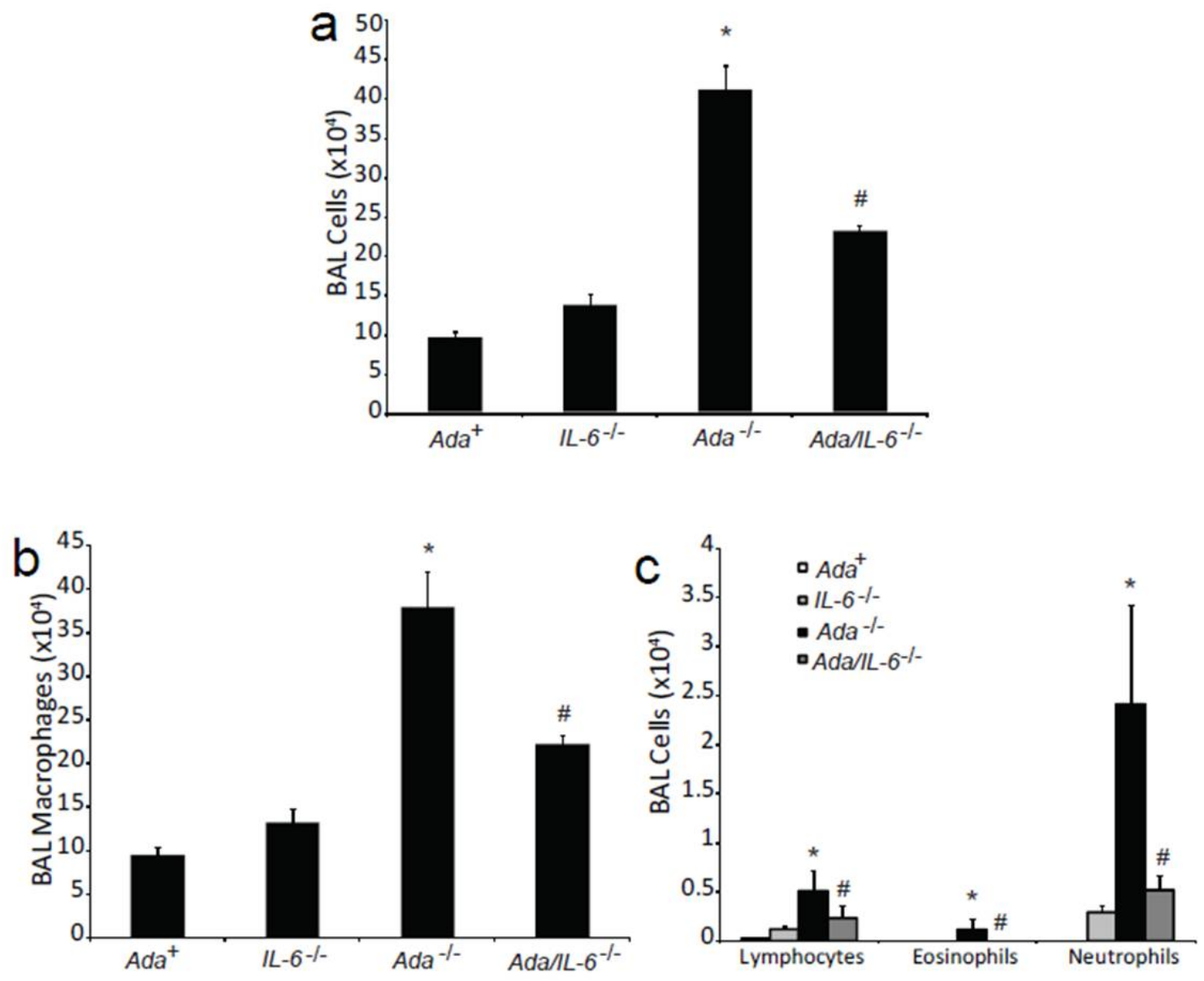

Figure 3.5

Pulmonary inflammation following genetic removal of IL-6 in ADA-deficient mice.

(a) Total cell numbers in BAL fluid were counted using a hemocytometer. BAL cells were cytospun and stained with Diff-Quick, allowing for quantification of macrophages (b) and lymphocytes, eosinophils, and neutrophils (c). Data are presented as mean cell counts \pm SEM, $n \geq 8 .\left({ }^{*}, p \leq 0.05\right.$ Ada $^{+}$vs. Ada ${ }^{--}$and \#, $\mathrm{p} \leq$ 0.05 Ada $^{-/-}$vs. Ada/lL- $6^{-/}$). 


\section{PULMONARY FIBROSIS IN Ada/IL-6 DOUBLE KNOCKOUT MICE}

In addition, Ada $^{-/-}$mice develop features of pulmonary fibrosis in particular collagen production and deposition (119). To determine the role of IL-6 on fibrosis in this model, Ada/IL- $6^{--}$mice were analyzed for fibrotic assessment. In this study, examination of metrics of pulmonary fibrosis revealed that Ada/IL- $6^{--}$ mice displayed reduced levels of collagen production (Fig 3.6a) and diminished myofibroblast accumulation $\alpha$-SMA (Fig 3.6b). This was supported by morphologic Ashcroft scores (Fig 3.6c). These results demonstrate that the removal of IL-6 is associated with diminished pulmonary fibrosis. Thus, this suggests that in the $A d a^{-/}$mice IL-6 is displaying a pro-fibrotic role by regulating key features of pulmonary fibrosis including collagen production and myofibroblast accumulation.

\section{ALVEOLAR DESTRUCTION IN Ada/IL-6 DOUBLE KNOCKOUT MICE}

Alveolar airspace enlargement is another feature of $A d a^{-/-}$mice that is a characteristic of emphysema and COPD. Ada/lL $-6^{/-}$mice were analyzed to determine whether IL-6 was involved in the regulation of airspace enlargement. The size of alveolar airspace was also diminished by the genetic removal of IL-6 (Fig 3.7a-b) in comparison with $\mathrm{Ada}^{-/-}$mice. This indicates that in the absence of IL-6 there is reduced alveolar destruction in the lungs of $A d a^{-/-}$mice. 


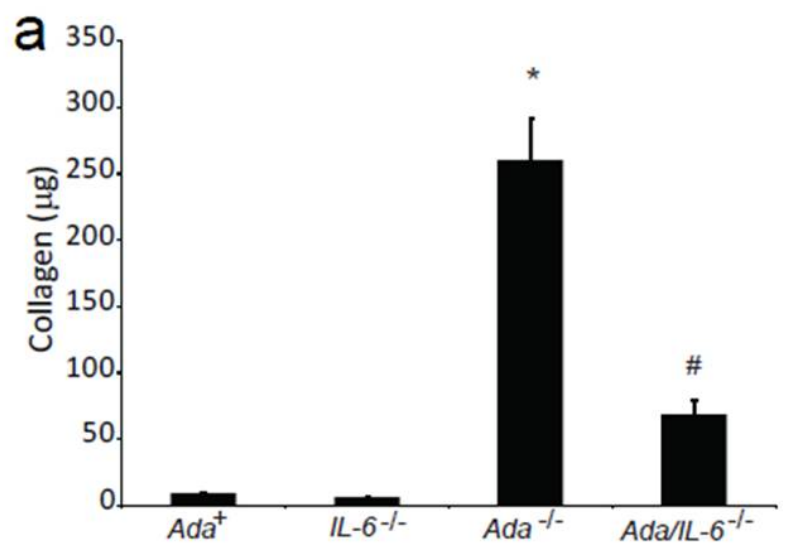

b

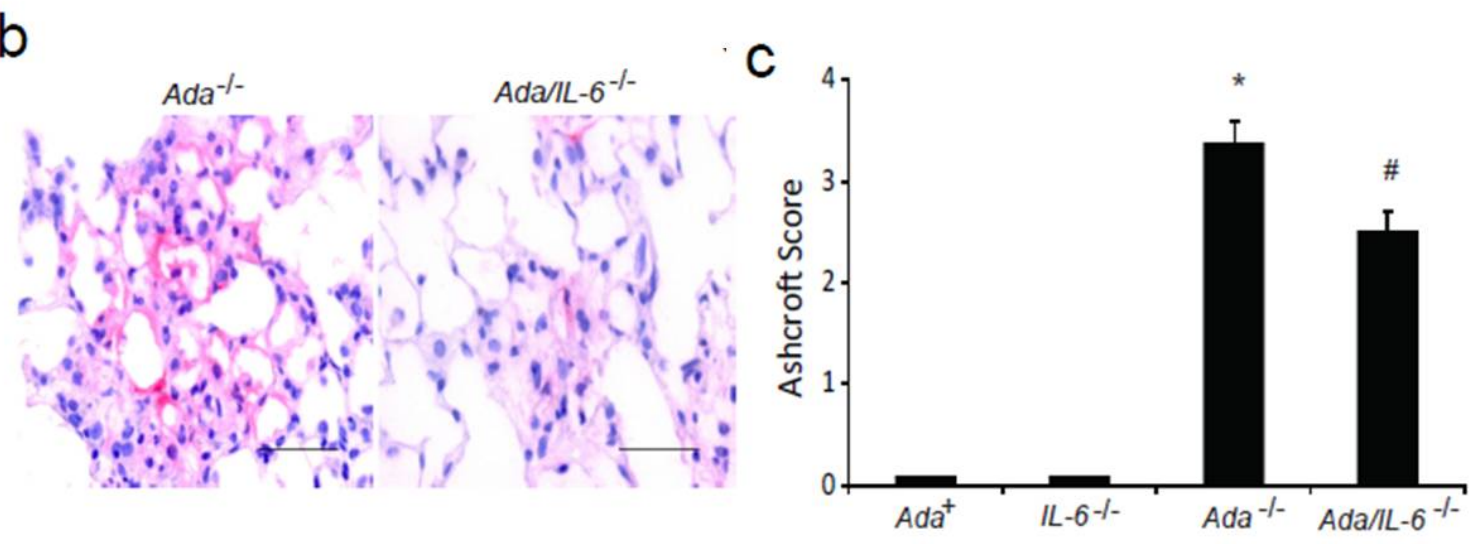

Figure 3.6

Pulmonary fibrosis following genetic removal of IL-6 in ADA-deficient mice.

Collagen production. (a) Soluble collagen levels were measured using the Sircol Assay. Data are presented as mean $\mu \mathrm{g}$ collagen $/ \mathrm{ml}$ BAL. Data are presented as mean cell counts \pm SEM, $n \geq 8$. ( ${ }^{*}, p \leq 0.05$ Ada $^{+}$vs. Ada ${ }^{-/}$and \#, $p \leq 0.05$ Ada $^{-/-}$ vs. $A d a /\left(L-\sigma^{\prime-}\right)$. (b) Lung sections were stained with an antibody against for $\alpha$ SMA to visualize myofibroblast accumulation (pink stain). Images are representative of 6 animals from each group. Scale bars: $100 \mu \mathrm{m}$. (c) Ashcroft scores were used to determine the degree of fibrosis ( ${ }^{*}, \mathrm{p} \leq 0.05 \mathrm{Ada}^{+}$vs. Ada ${ }^{-{ }^{-}}$ and \#, $\mathrm{p} \leq 0.05$ Ada $^{-/-}$vs. Ada/lL-6 ${ }^{-/-}, \mathrm{n} \geq 6$ ). 

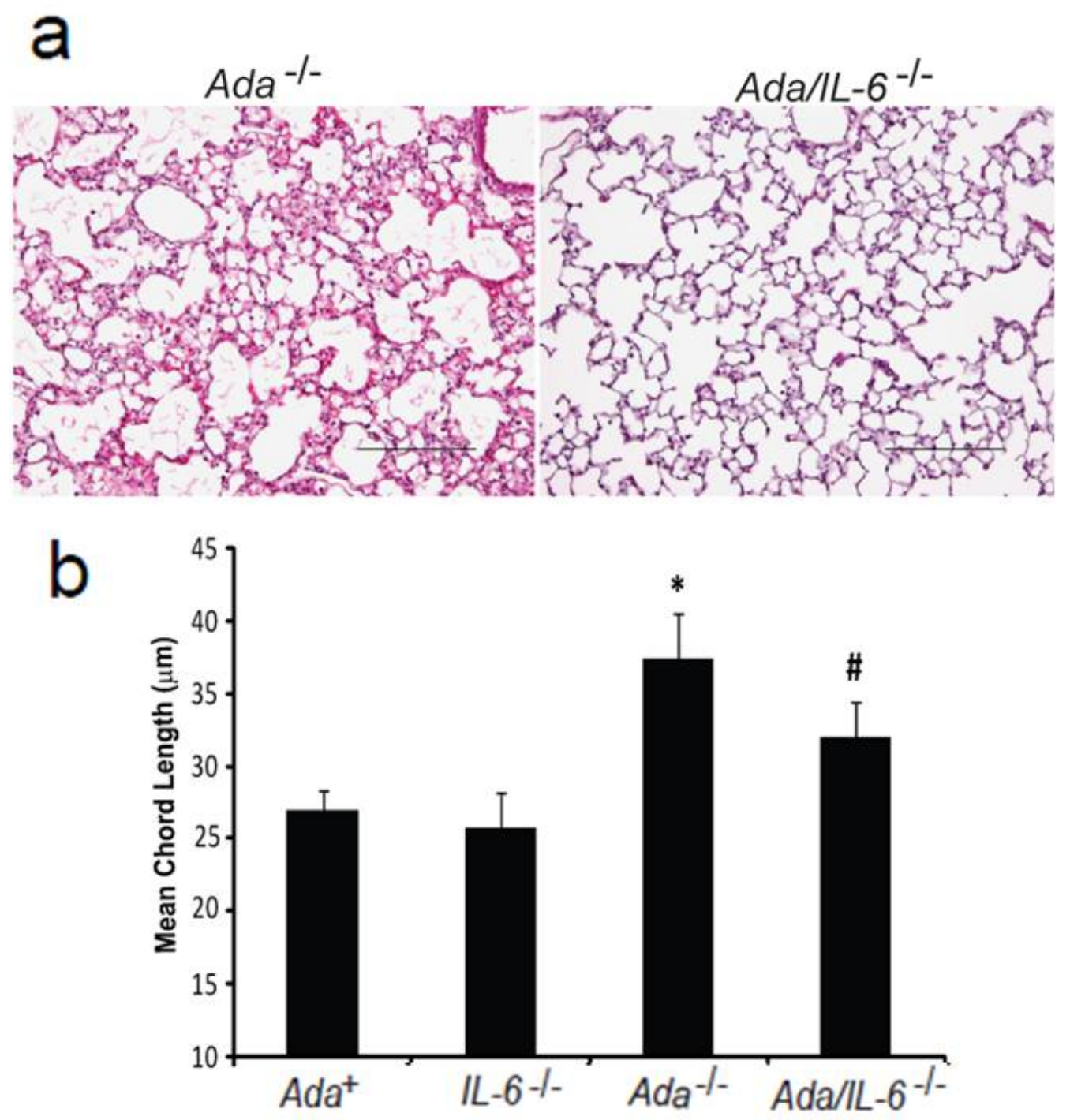

Figure 3.7

\section{Alveolar destruction following genetic removal of IL-6 in ADA-deficient mice.}

(a) Histology of the lungs from postnatal day $43 \mathrm{Ada}^{+}, \mathrm{Ada}^{-{ }^{-/}}$, and $\mathrm{Ada} / \mathrm{IL}-6^{-/}$mice revealing diminished alveolar airspace destruction. Images are representative of 8 animals from each group. Scale bars: $200 \mu \mathrm{m}$. (b) Quantitative analysis of alveolar airspace size calculated using Image-Pro analysis software. Values are presented as mean chord lengths in $\mu \mathrm{m} \pm \mathrm{SEM}, \mathrm{n} \geq 8$. ( ${ }^{*}, \mathrm{p} \leq 0.05 \mathrm{Ada}^{+}$vs. Ada ${ }^{-/}$ and \#, $\mathrm{p} \leq 0.05 \mathrm{Ada}^{-{ }^{-}}$vs. Ada/lL-6/-). 


\section{STAT-3 ACTIVATION IN EPITHELIAL CELLS IN ADA-DEFICIENT MICE FOLLOWING GENETIC REMOVAL OF IL-6}

STAT-3 is a well known target of IL-6 (90). Examination of the status of STAT-3 activation reveals that $A d{ }^{-/-}$mice display an enhanced phosphorylation of STAT-3. However, Ada/IL- $6^{--}$mice exhibit reduced STAT-3 activation (Fig 3.8a-b). Immunolocalization of phospho-STAT-3 revealed a reduction of nuclear staining in alveolar epithelial cells of $A d a / I L-6^{--}$mice (Fig. 3.8c). These results suggest that in the Ada $^{-/}$mice, IL-6 promotes STAT-3 activation in airway epithelial cells and contributes to pulmonary inflammation, airspace destruction and fibrosis.

\section{IL-6 NEUTRALIZATION IN ADA-DEFICIENT MICE IS ASSOCIATED WITH DECREASED PULMONARY INFLAMMATION, FIBROSIS, AND STAT-3 ACTIVATION. \\ PULMONARY PATHOLOGY IN ADA-DEFICIENT MICE FOLLOWING IL-6 NEUTRALIZATION}

Results in Ada/lL- $6^{--}$mice suggest that IL-6 targeted therapies may be useful in the treatment of adenosine-mediated pulmonary inflammation and fibrosis. To assess this further, $A d a^{-/-}$mice were treated systemically with an IL-6 neutralizing antibody (Fig 3.3). Treatment of $A d a^{-/-}$mice with the IL-6 neutralizing antibodies was performed via subcutaneous injections at postnatal day 26,31 , and 37. The exposure levels of the anti-IL-6 antibody were determined by ELISA in the plasma and BAL fluid (Fig 3.10). No significant difference was observed on the plasma levels between the $A_{d a}^{+}$mice and $A d a^{-/-}$mice $(\sim 300 \mu \mathrm{g} / \mathrm{ml})$, suggesting consistent exposure. Whereas in the lung, anti-IL-6 exposure levels 

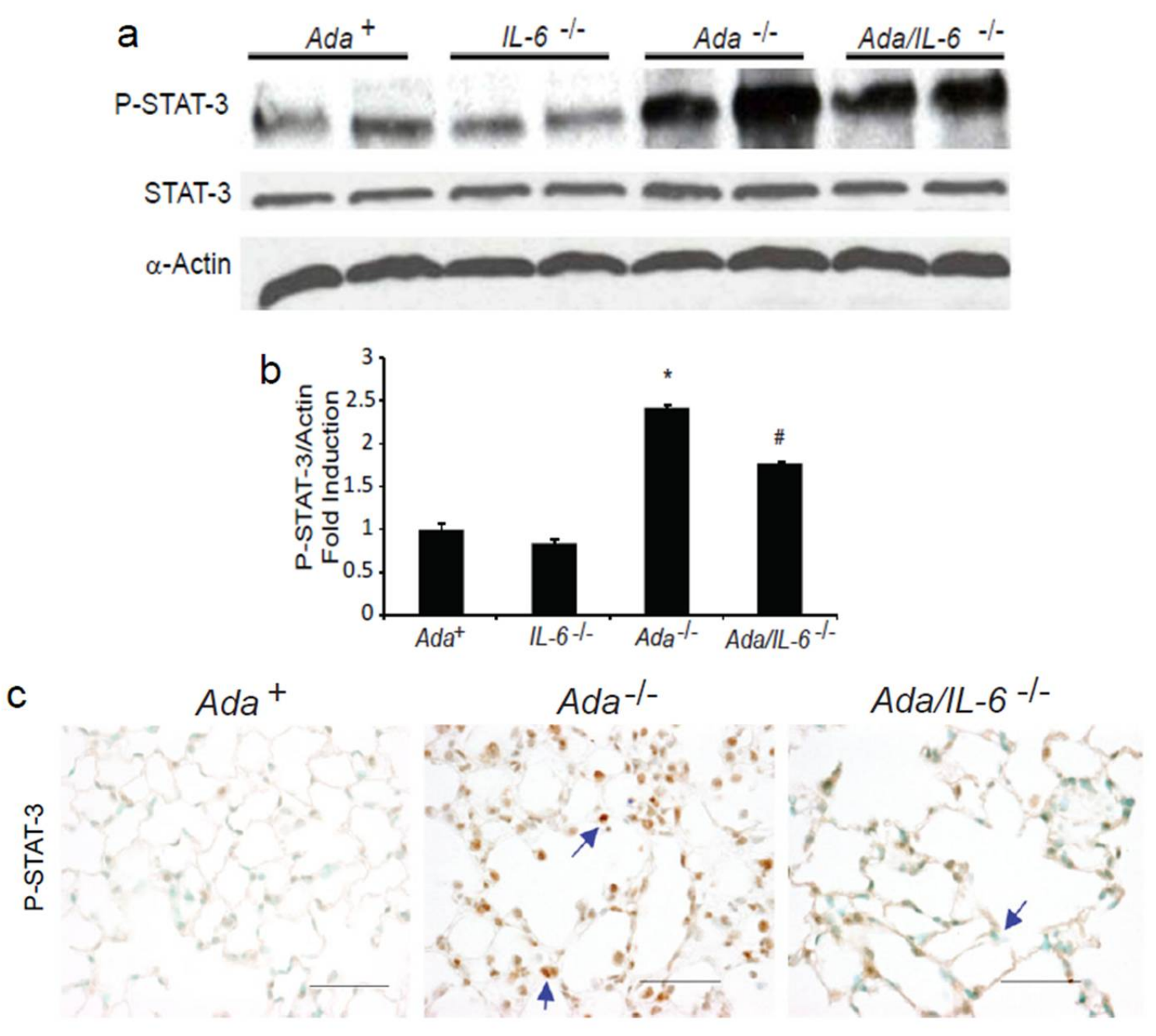

Figure 3.8

STAT-3 activation following genetic removal of IL-6 in ADA-deficient mice.

(a) Phospho STAT-3 activation. Western Blot analysis was performed using an antibody against phospho-STAT-3 in whole lungs lysate from postnatal day 43 $\mathrm{Ada}^{+}$and Ada/lL- $6^{/-}$mice. STAT-3 and $\alpha$-actin were used as controls. (b) Phospho-STAT-3 band intensity was quantified using ImageJ analysis. Values are presented as the percentage of $\alpha$-actin \pm SEM, $n \geq 4$. $\left({ }^{*}, p \leq 0.05\right.$ Ada $^{+}$vs. $\mathrm{Ada}^{-/-}$and \#, $\mathrm{p} \leq 0.05 \mathrm{Ada}^{-/-}$vs. Ada/lL-6 ${ }^{-/}$). (c) Immunolocalization of phosphoSTAT-3 expression. Lung sections were stained with an antibody specific for phospho-STAT-3 on alveolar epithelial cells (blue arrows). Images are representative of 8 animals from each group. Scale bars: $100 \mu \mathrm{m}$. 
in the BAL fluid were significantly different between the Ada $^{+}$mice $(0.23 \mu \mathrm{g} / \mathrm{ml})$ and $A_{d a} a^{--}$mice $(1.93 \mu \mathrm{g} / \mathrm{ml})$. This may be due to the increased vascular barrier disruption observed in the $A d a^{-/-}$mice. Nonetheless, the similar levels in the plasma from $\mathrm{Ada}^{+}$and $\mathrm{Ada}^{-/-}$mice suggest that adequate anti-IL-6 exposure is achieved. Examination of lung histology through H\&E staining revealed that Ada 1- mice treated with the isotype antibody developed severe inflammation and damage. Conversely, Ada $^{-/-}$mice treated with the anti-IL-6 antibody displayed a reduction in lung inflammation, air-space enlargement, and fibrosis (Fig 3.9). These findings suggest that IL-6 contributes to the development and progression of pulmonary inflammation and fibrosis in the $A d a^{-/}$mice and that IL-6 based therapeutic approach may provide benefits in adenosine-driven lung disease.

\section{PULMONARY INFLAMMATION IN ADA-DEFICIENT MICE FOLLOWING IL-6 NEUTRALIZATION}

To quantify the reduced pulmonary inflammation observed on histologic findings, BAL was performed and recovered inflammatory cells were counted using a hemocytometer. Results revealed characteristic increases in total inflammatory cells recovered from BAL fluid of $\mathrm{Ada}^{-/}$mice versus $\mathrm{Ada}^{+}$mice, but a significant reduction was observed in $A d a^{-/-}$mice treated with IL-6 neutralizing antibodies (Fig 3.11a). Analysis of BAL cellular differentials revealed that IL-6 neutralization led to a significant reduction in all cell types examined including macrophages (Fig 3.11b), lymphocytes, eosinophils, and neutrophils (Fig 3.11c). These data suggest that IL-6 contributes to pulmonary inflammation in $\mathrm{Ada}^{-/}$ mice. 


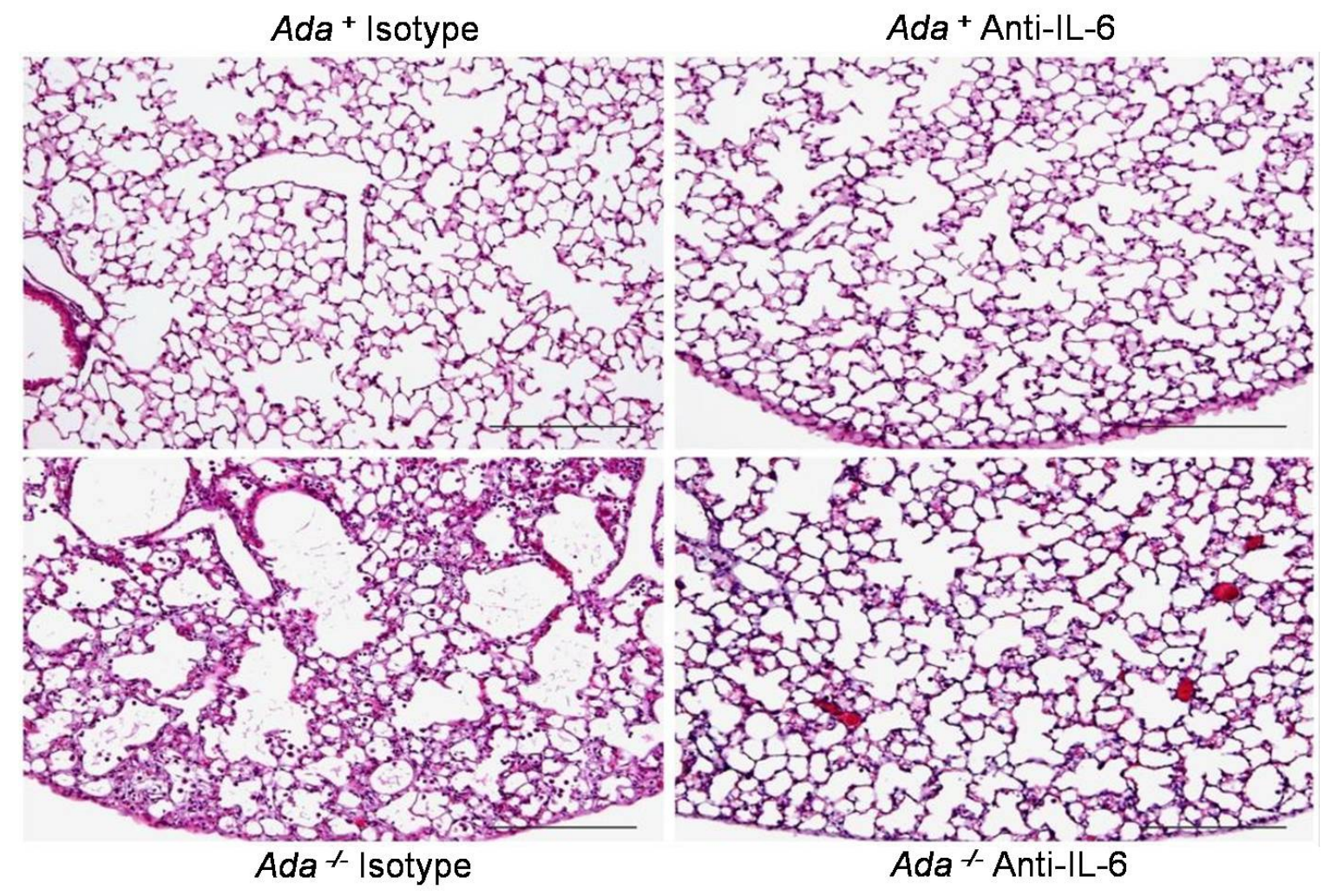

Figure 3.9

\section{Pulmonary phenotypes following neutralization of IL-6 in ADA-deficient mice.}

Lungs from postnatal day $43 \mathrm{Ada}^{+}$mice treated with the isotype antibody (upper left) or IL-6 antibody (upper right) and $A d a^{-/-}$mice treated with the isotype antibody (lower left) or IL-6 antibody (lower right) were infused with fixative under constant pressure $\left(25 \mathrm{~cm} \mathrm{H}_{2} \mathrm{O}\right)$ and processed for H\&E staining. Assessment of lung inflammation and damage in $\mathrm{Ada}^{-/}$mice following subcutaneous injections with an anti IL-6 antibody ( $30 \mathrm{mg} / \mathrm{kg}$ ) displayed a reduction in lung inflammation, airway enlargement, and fibrosis. Images are representative of 11 animals from each group. Scale bars: $200 \mu \mathrm{m}$. 


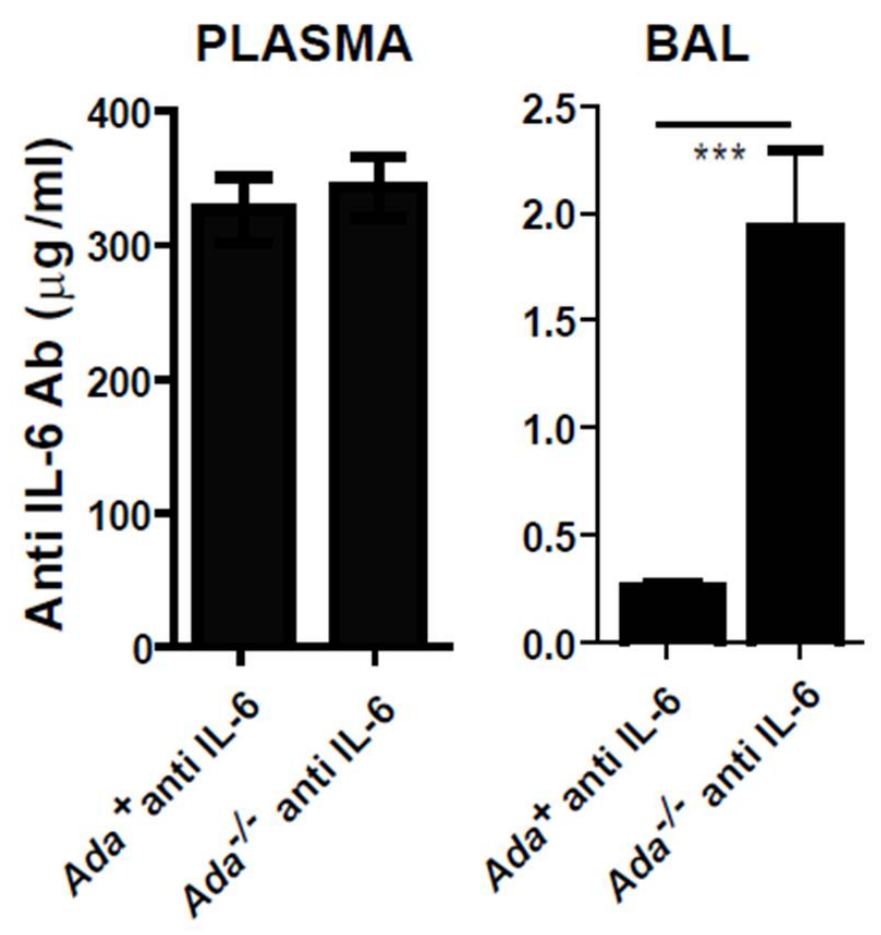

Figure 3.10

Anti IL-6 exposure in ADA-deficient mice.

PK analysis revealing the IL-6 antibody exposure levels in the plasma and BAL. Data are presented as mean $\mu \mathrm{g} / \mathrm{ml}$ IL-6 antibody \pm SEM, $n \geq 11 . \quad\left({ }^{* * *} p<0.001\right.$ using one way ANOVA and Bonferonnis Multiple comparison test). 

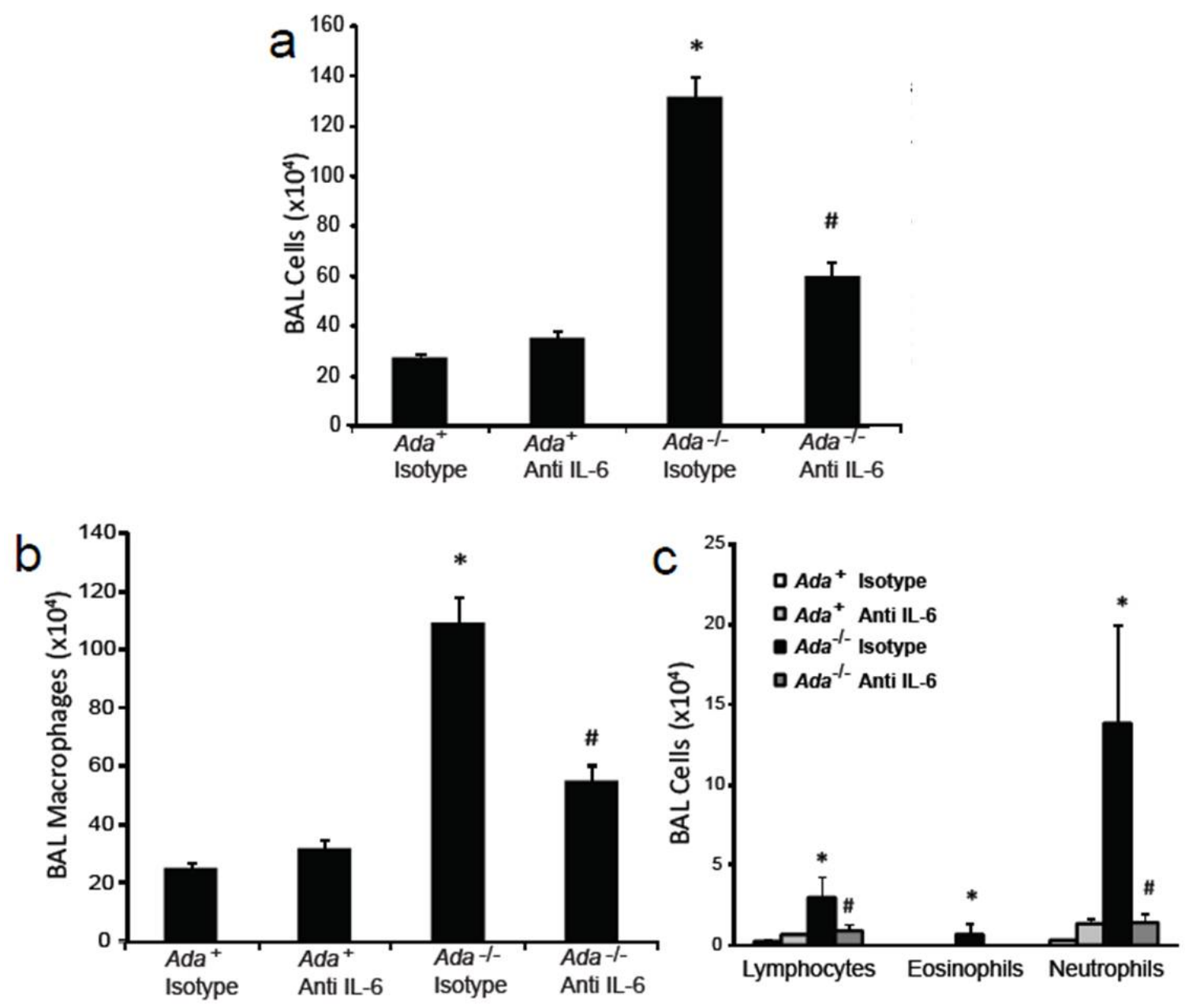

Figure 3.11

\section{Pulmonary inflammation following neutralization of IL-6 in ADA-deficient mice.}

Bronchoalveolar lavage (46) was performed on postnatal day $43 \mathrm{Ada}^{+}$or $\mathrm{Ada}^{-1-}$ mice treated with the isotype or IL-6 antibody. (a) Total cells numbers in lavage fluid were counted using a hemocytometer. BAL cells were cytospun and stained with Diff-Quick, allowing for determination of macrophages (b) and lymphocytes, eosinophils, and neutrophils (c). Data are presented as mean cell counts \pm SEM, $n \geq 11$. ( ${ }^{*}, \mathrm{p} \leq 0.05 \mathrm{Ada}^{+}$vs. $\mathrm{Ada}^{-/-}$and \#, $\mathrm{p} \leq 0.05 \mathrm{Ada}^{-/-}$vs. Ada ${ }^{-/-}+$ IL-6 Antibody). 


\section{PULMONARY FIBROSIS IN ADA-DEFICIENT MICE FOLLOWING IL-6 NEUTRALIZATION}

In conjunction with increases in tissue levels of adenosine, Ada ${ }^{-/-}$mice develop features of pulmonary fibrosis including the production and deposition of collagen $(79,119,136)$. We next sought to determine if treatment with IL-6 neutralizing antibodies had a therapeutic effect on the pulmonary fibrosis seen in $A d a^{-/}$mice. Collagen production and deposition were examined to determine the effect of IL-6 neutralization on pulmonary fibrosis in this model. Treatment of $A_{d a} a^{-/}$mice with IL-6 neutralizing antibodies resulted in reduced soluble collagen levels in BAL fluid (Fig 3.12a). Furthermore, examination of a1-procollagen transcript levels (Fig 3.12b) and airway collagen deposition (Fig 3.13b) revealed enhanced collagen production in the lungs of $\mathrm{Ada}^{-/-}$mice that were attenuated following anti-IL-6 treatment. Staining for $\alpha$-SMA revealed prominent myofibroblast accumulation in the distal airways of $A d a^{-/-}$mice (Fig 3.12c). This accumulation was largely prevented in $A d a^{-/}$mice following IL-6 neutralization. Thus, in anti-IL-6-treated $A d a^{-/-}$mice, pulmonary fibrosis was attenuated as indicated by the reduction of key fibrotic indexes including Ashcroft scores (Fig 3.12d). In addition, the production of fibronectin, an extracellular matrix protein involved in inflammation, airspace destruction, and pulmonary fibrosis (39), was examined in Ada $^{-/-}$mice treated with isotype or IL-6 neutralizing antibodies. Ada I- mice exhibited an increase in fibronectin production and deposition that was diminished by IL-6 neutralization (Fig $3.13 a, c)$. Collectively, these findings indicate that in $A d a^{-/-}$mice, IL-6 signaling is involved in regulating the production 

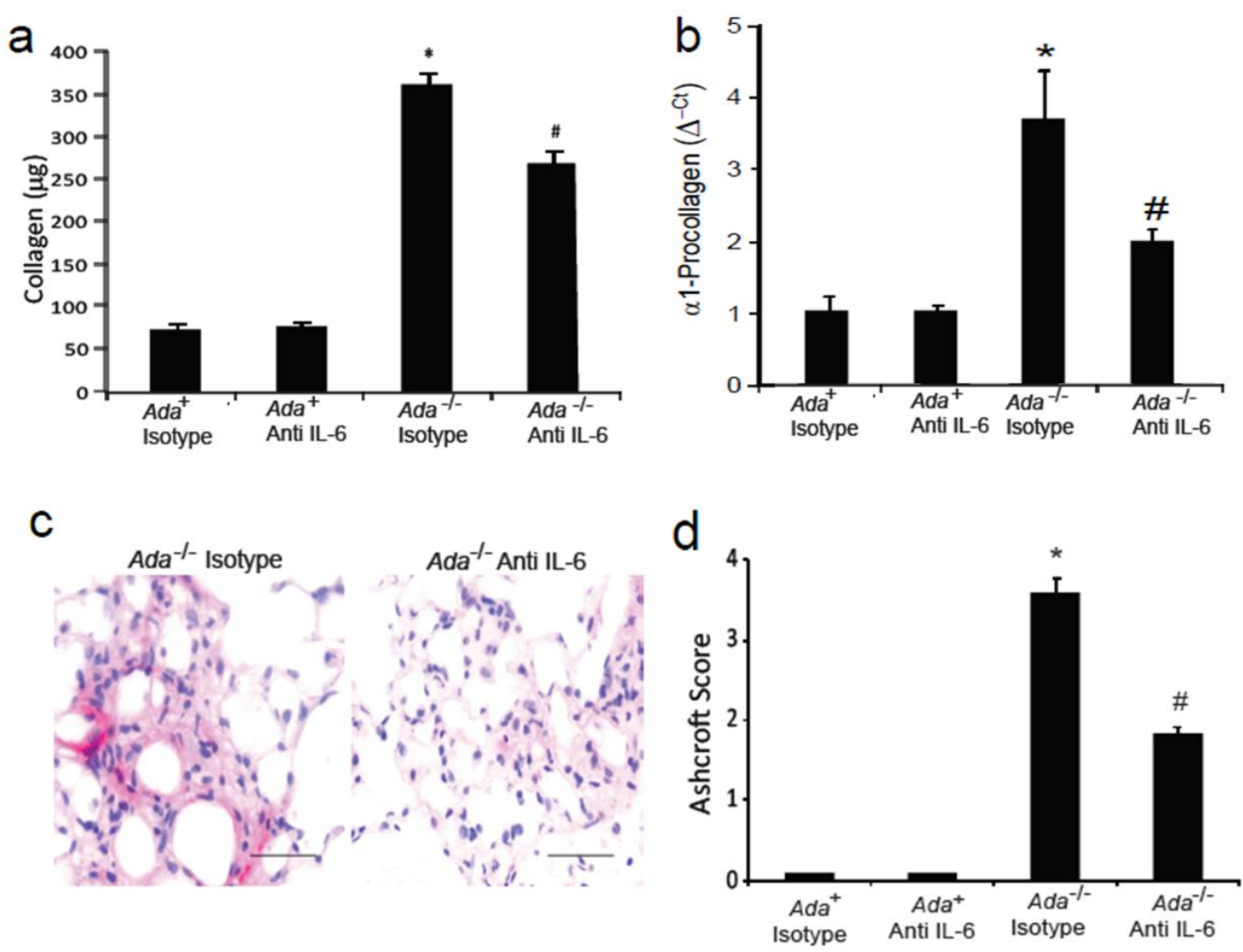

Figure 3.12

\section{Pulmonary fibrosis following neutralization of IL-6 in ADA-deficient mice.}

Fibrotic characteristics were assessed from lung sections from postnatal day 43 $\mathrm{Ada}^{+}$mice treated with the isotype antibody, $\mathrm{Ada}^{-/-}$mice treated with the isotype antibody or $A d a^{-/-}$mice treated with the IL-6 antibody. (a) Decreased collagen levels. Soluble collagen protein levels were measured using Sircol Assay. Data presented as mean $\mu \mathrm{g}$ collagen $/ \mathrm{ml} \mathrm{BAL} \mathrm{fluid} \pm$ SEM, $\mathrm{n} \geq 8$. ( ${ }^{*}, \mathrm{p} \leq 0.05 \mathrm{Ada}^{+}$vs. Ada $^{-/-}$and \#, p $\leq 0.05$ Ada $^{-/-}$vs. Ada ${ }^{-/-}$IL-6 Antibody). (b) Decreased collagen production. Whole-lung $\alpha 1$-procollagen transcript levels measured using quantitative RT-PCR. Data are presented as mean normalized 18S rRNA transcript levels $\left(\Delta^{-\mathrm{ct}}\right) \pm \mathrm{SEM}, \mathrm{n} \geq 4 .{ }^{*}{ }^{*}, \mathrm{p} \leq 0.05 \mathrm{Ada}^{+}$vs. $\mathrm{Ada}^{-/-}$and $\#, \mathrm{p} \leq 0.05$ Ada $^{-/-}$vs. Ada ${ }^{-/-}$IL-6 Antibody). (c) Decreased myofibroblast accumulation. Lung sections were stained with an antibody against for $\alpha$-SMA to visualize myofibroblast (pink stain). Images are representative of 6 animals from each group. Scale bars: $100 \mu \mathrm{m}$. (d) Ashcroft scores were used to determine the degree of fibrosis ( ${ }^{*}, \mathrm{p} \leq 0.05 \mathrm{Ada}^{+}$vs. $\mathrm{Ada}^{-/-}$and \#, $\mathrm{p} \leq 0.05 \mathrm{Ada}^{-/-}$vs. Ada ${ }^{-/-}+\mathrm{IL}-$ 6 Antibody, $\mathrm{n} \geq 6$ ). 

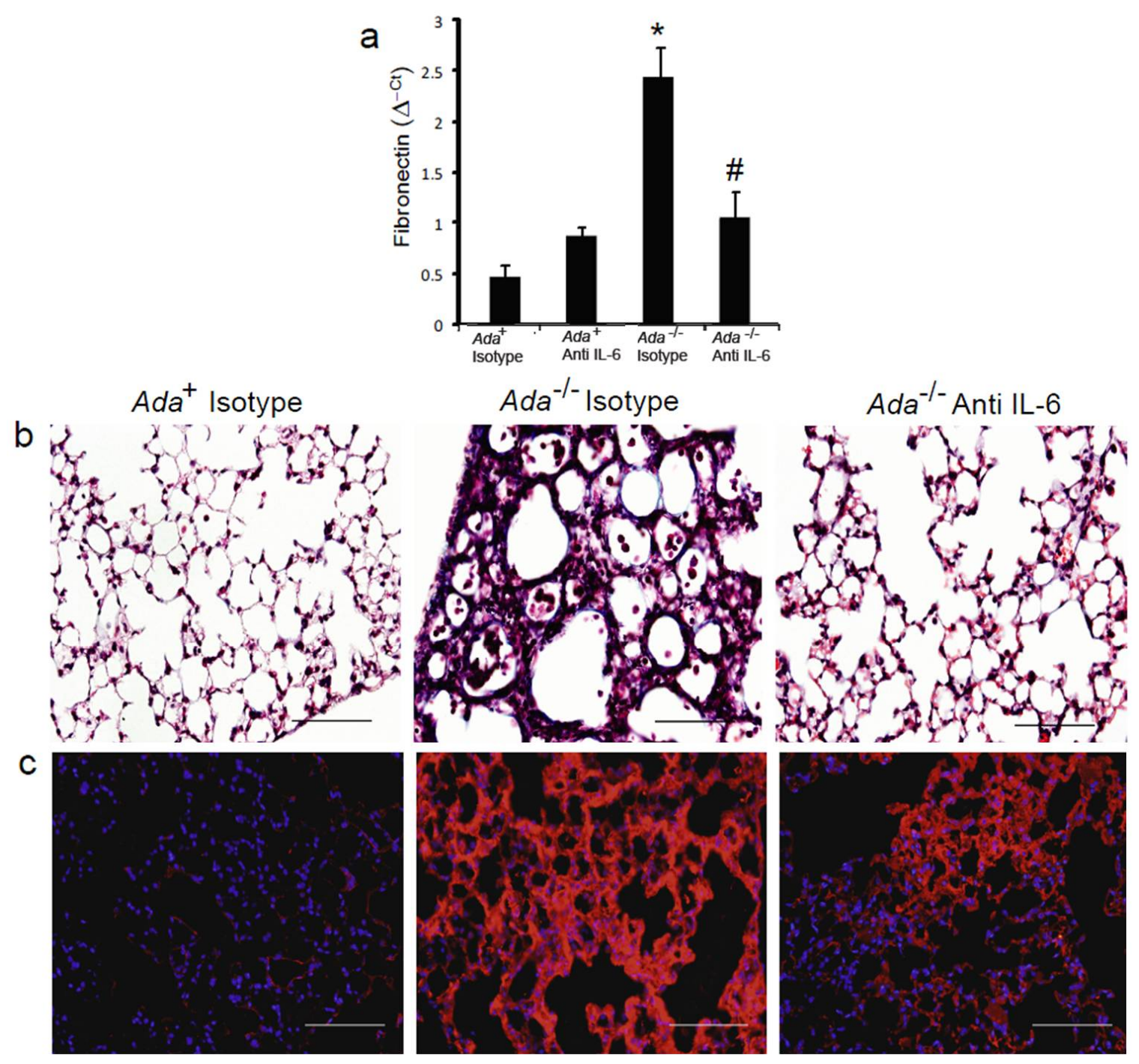

Figure 3.12

\section{IL-6 neutralization in ADA-deficient mice is associated with decreased pulmonary fibrosis.}

(a) Decreased fibronectin production. Whole-lung fibronectin transcript levels measured using RT-PCR. Data presented as mean normalized 18S rRNA transcript levels $\left(\Delta^{-c t}\right) \pm$ SEM, $n \geq 4 .\left({ }^{*}, p \leq 0.05\right.$ Ada $^{+}$vs. Ada ${ }^{-/}$and $\#, p \leq 0.05$ Ada $^{-\gamma^{-}}$vs. Ada ${ }^{-/-}$+ IL-6 Antibody). (b) Decreased collagen deposition. Lung sections were stained with Masson's Trichome to visualize collagen deposition (blue stain). (c) Decreased fibronectin deposition. Lung sections were treated with a specific fibronectin antibody (red stain). Images are representative of 8 animals from each group. Scale bars: $200 \mu \mathrm{m}$. 
and deposition of collagen and fibronectin and in the regulation of myofibroblasts accumulation, common features seen in the progression of pulmonary fibrosis.

\section{INFLAMMATORY MEDIATORS IN ADA-DEFICIENT MICE FOLLOWING IL-6 NEUTRALIZATION}

To examine potential mechanisms by which IL-6 neutralization causes diminished pulmonary inflammation in $A d a^{-/}$mice, cytokine and chemokine transcript levels were measured in whole lung RNA extracts. Results demonstrated characteristic increases of proinflammatory cytokine expression in the lungs of $A d a^{-/}$mice, but a significant reduction was observed in cytokines and chemokines known to be IL-6 target (Fig 3.13). Among the proinflammatory cytokines and chemokines found to be reduced via IL-6 neutralization were CXCL1, CCL2 (MCP-1), osteopontin (OPN), IL-17, and CXCL-2 (MIP-2). In contrast, expression of TNF- $\alpha$ and TGF- $\beta$ (data not shown) were found to be elevated in the lungs of $\mathrm{Ada}^{-/-}$mice treated with isotype antibodies; however, their expression was not reduced following IL-6 neutralization. These findings suggest that IL-6 significantly influences the expression of certain proinflammatory cytokines and chemokines, which are known mediators that contribute to the pulmonary inflammation observed in the lungs of $\mathrm{Ada}^{-/-}$mice.

\section{MEDIATORS OF ALVEOLAR DESTRUCTION IN ADA-DEFICIENT MICE FOLLOWING IL-6 NEUTRALIZATION}

ADA-deficient display features of alveolar airspace enlargement resembling emphysema (79). To determine the effects of IL-6 neutralization on 

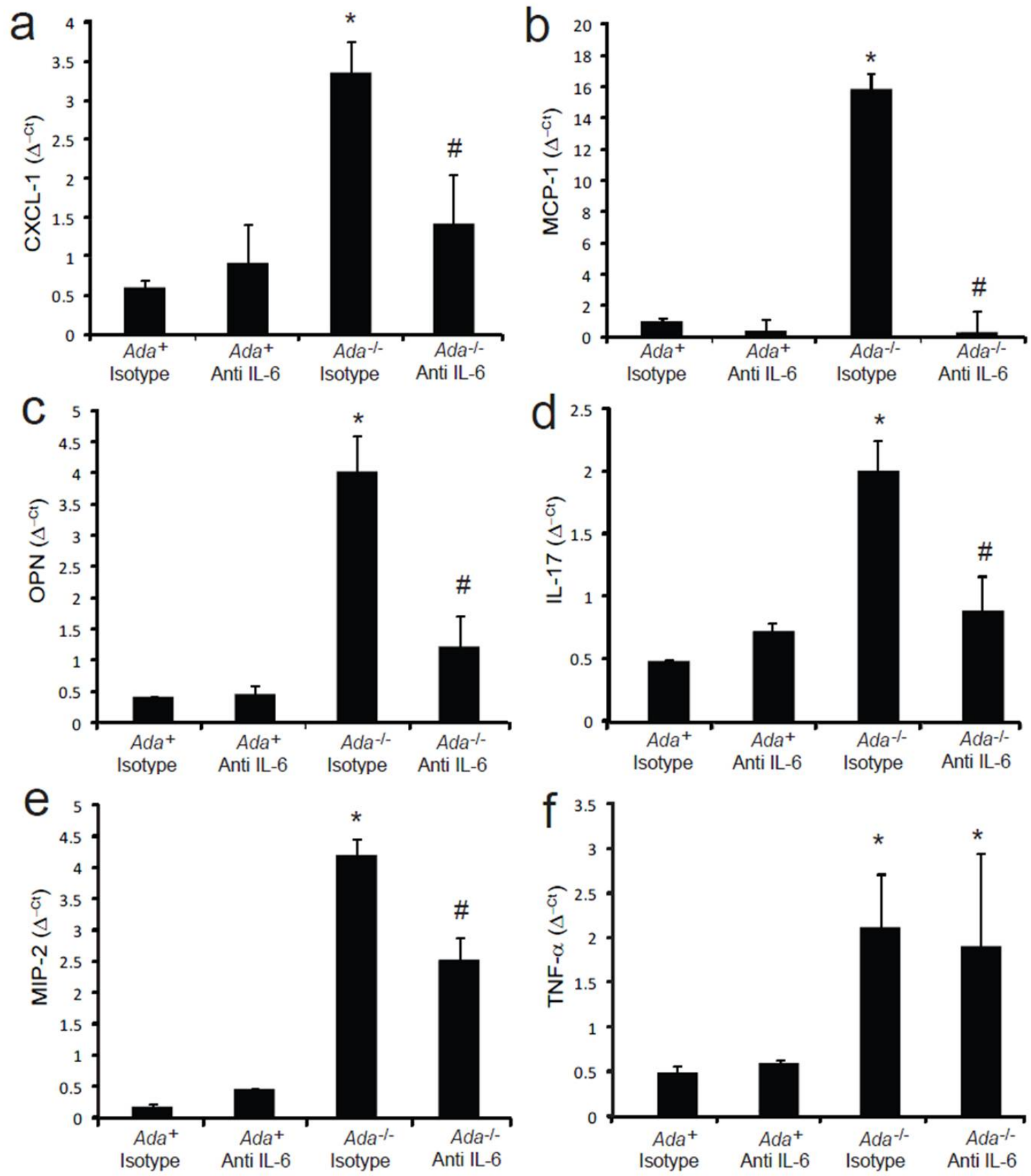

Figure 3.13

\section{Proinflammatory mediators in mice treated with IL-6 neutralizing} antibodies.

Transcript levels of various proinflammatory cytokines and chemokines were measured in whole-lung RNA extracts from postnatal day 43 mice using quantitative RT-PCR. Shown are levels of (a) CXCL-1, (b) CCL2 [MCP-1], (c) Osteopontin [OPN], (d) IL-17, (e) CXCL2 [MIP-2], and (f) TNF- $\alpha$. Transcripts were measured in parallel with 18S rRNA and values are presented as mean normalized transcript levels $\left(\Delta^{-\mathrm{ct}}\right) \pm \mathrm{SEM}, \mathrm{n} \geq 4 .{ }^{*}, \mathrm{p} \leq 0.05 \mathrm{Ada}^{+}$vs. Ada ${ }^{-/-}$and \#, $\mathrm{p}$ $\leq 0.05$ Ada $^{-/-}$vs. Ada ${ }^{-/-}+$IL-6 Antibody). 
this phenotype, mean chord length analysis was conducted on lungs from $\mathrm{Ada}^{-/-}$ mice treated with IL-6 neutralizing antibodies. Results demonstrated that the enlarged airspaces observed in the $A d a^{-/}$mice were prevented with treatment with anti-IL-6 antibodies (Fig 3.14a, b). These data demonstrate that alveolar destruction in the lungs of $A d a^{-/-}$mice can be prevented via IL-6 neutralization.

Alveolar airspace enlargement is characterized by a disruption in the balance between the levels of MMPs and inhibitors of proteases (79). Ada ${ }^{-/-}$mice exhibits increased expression of tissue inhibitor of metalloproteinase-1 (TIMP-1), MMP-9, and MMP-12 (79). Examination of these mediators in the lungs of Ada mice treated with IL-6 neutralizing antibodies revealed diminished expression of TIMP-1 and MMP-9, while MMP-12 levels remained elevated (Fig 3.15a-d). MMP-9 immunostaining confirmed increased MMP-9 protein levels in inflammatory cells in the lungs of $A d a^{-/-}$mice, and significant reductions following IL-6 neutralization (Fig 3.15e). These results suggest that IL-6 signaling is involved in the regulation of alveolar airspace destruction by the regulation of these key regulators.

\section{VASCULAR PERMEABILITY IN ADA-DEFICIENT MICE FOLLOWING IL-6 NEUTRALIZATION}

Previous studies indicate that $A_{2 B} R$ signaling preserves endothelial barrier function during hypoxia and lung injury $(129,137)$, and $A_{2 B} R$ deficiency enhances vascular leakage in $A_{d a}^{-/-}$mice . In the setting of elevated adenosine levels, $A_{2 B} R$ regulates cytokine production including IL-6 (79). Furthermore, excess IL-6 produces gastrointestinal barrier dysfunction by downregulating tight junction 
a

$\mathrm{Ada}^{+}$Isotype
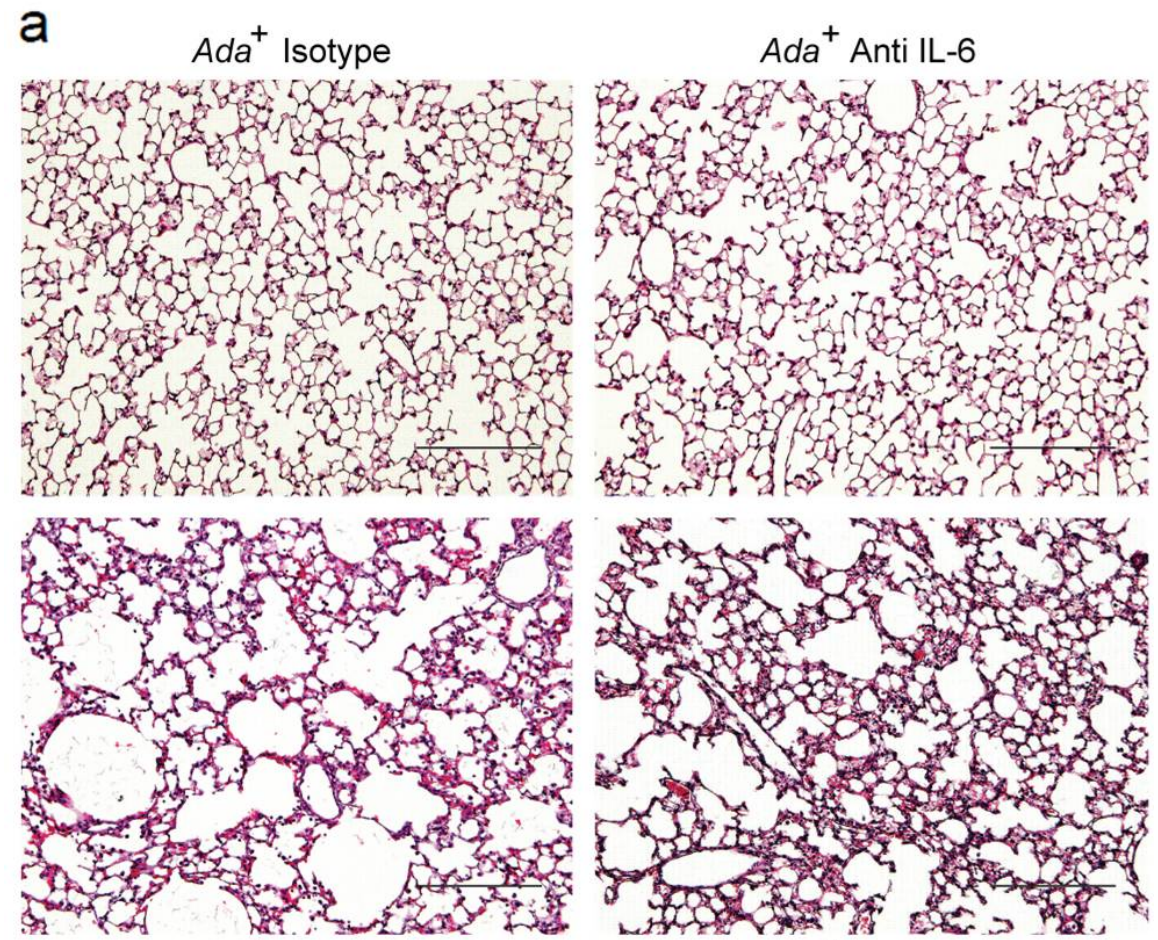

Ada $^{-/-}$Isotype

$$
\text { Ada }{ }^{-/-} \text {Anti IL-6 }
$$

b

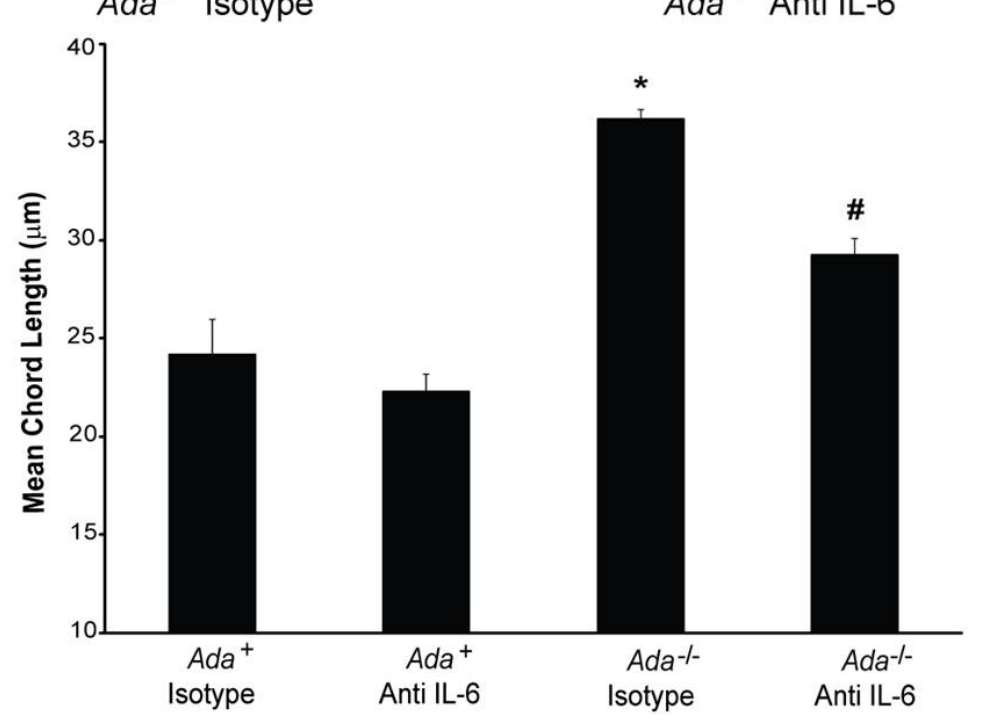

Figure 3.14

Alveolar destruction following neutralization of IL-6 in ADA-deficient mice.

(a) Histological examination of $\mathrm{H} \& \mathrm{E}$ lung sections from postnatal day $43 \mathrm{Ada}^{+}$ mice treated with the isotype antibody or IL-6 antibody and $A d a^{-/-}$mice treated with isotype antibody or IL-6 antibody (lower right). Images are representative of 11 animals from each group. Scale bars: $200 \mu \mathrm{m}$. (b) Quantitative analysis of alveolar airspace size calculated using Image-Pro analysis software. Values are presented as mean chord lengths in $\mu \mathrm{m} \pm \mathrm{SEM}, \mathrm{n} \geq 11$. $\left({ }^{*}, \mathrm{p} \leq 0.05\right.$ Ada+ vs. Ada ${ }^{-/}$ and \#, p $\leq 0.05$ Ada $^{-/-}$vs. Ada ${ }^{--}+$IL-6 Antibody). 

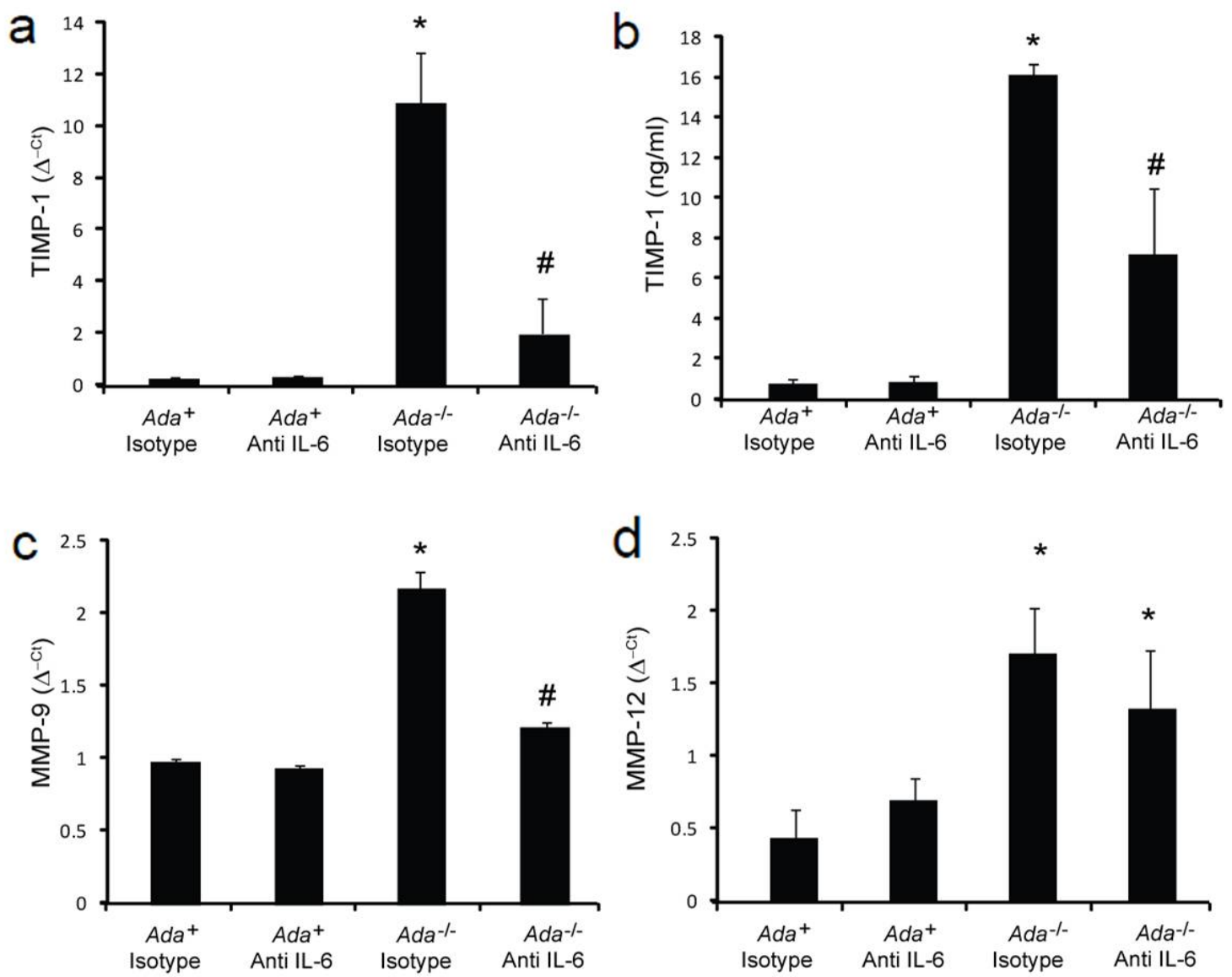

e
$\mathrm{Ada}^{+}$Isotype
Ada ${ }^{-/-}$Isotype
Ada ${ }^{-/-}$Anti IL-6
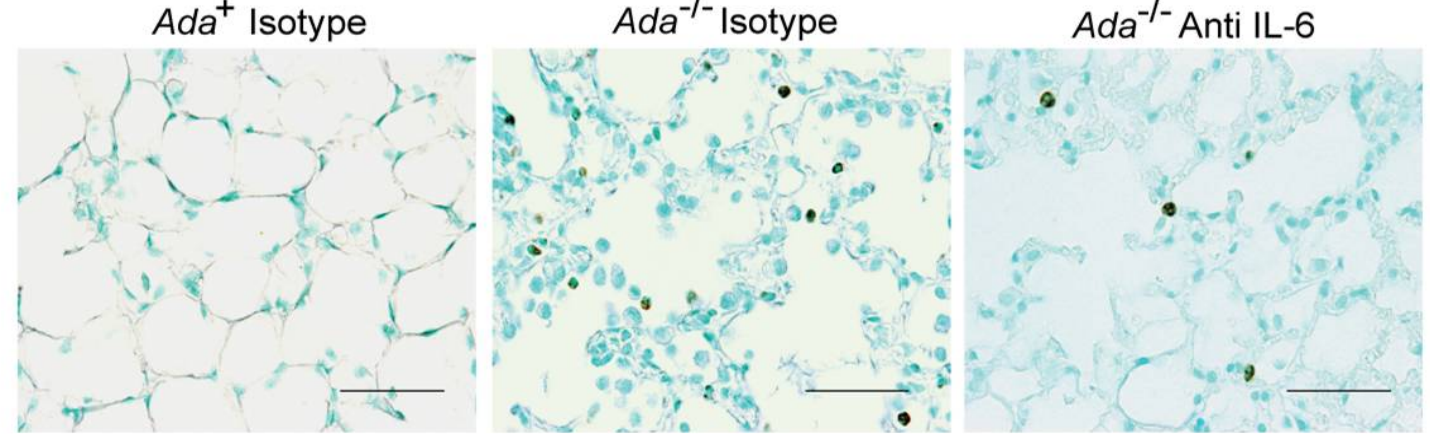

Figure 3.15

Alveolar destruction mediators in mice treated with IL-6 neutralizing antibodies. Transcript levels of TIMP-1 (a), MMP-9 (c), and MMP-12 (d) measured from whole-lung RNA extracts using RT-PCR. Transcripts were measured in parallel with 18S rRNA and values are presented as mean normalized transcript levels $\left(\Delta^{\text {ct }}\right) \pm S E M, n \geq 4$. (b) TIMP-1 protein levels were measured in BAL fluid using an ELISA kit. Values are presented as $\mathrm{ng} / \mathrm{ml} \pm$ SEM, $\mathrm{n} \geq 6$. ( ${ }^{*}, \mathrm{p} \leq 0.05 \mathrm{Ada}^{+}$vs. Ada ${ }^{-/-}$and \#, $\mathrm{p} \leq 0.05 \mathrm{Ada}^{-/-}$vs. Ada ${ }^{-/-}+\mathrm{IL}-6$ Antibody). (e) MMP-9 immunolocalization in Ada $^{-/-}$mice. Images are representative of 8 animals from each group. Scale bars: $200 \mu \mathrm{m}$. 
protein metabolism (138). Hence, to address the role of IL-6 on endothelial barrier function, $A d a^{-/}$mice treated with an anti-IL-6 antibody or isotype antibody were subjected to comparative analysis of vascular permeability as assessed by Evans blue dye extravasation. Results indicated a significant increase in vascular leakage in lungs of $A d a^{-/-}$mice. This increase was prevented by the treatment of $A d a^{-/-}$mice with IL-6 neutralizing antibodies (Fig $3.16 \mathrm{a}, \mathrm{b}$ ). Hence, neutralization of IL-6 is associated with restored vascular permeability in $\mathrm{Ada}^{-/-}$ mice. These findings indicate that IL-6 serves as an important mediator for pulmonary endothelial barrier function in $\mathrm{Ada}^{-/-}$mice.

\section{MUCUS CELL METAPLASIA IN ADA-DEFICIENT MICE FOLLOWING IL-6 NEUTRALIZATION}

Elevations of adenosine in the airways of $\mathrm{Ada}^{-/-}$mice are observed in conjunction with prominent features of mucous cell metaplasia in the bronchial airways (79). To determine the necessity of IL-6 in mucin production in this setting of increased adenosine levels, $A d a^{-/-}$mice were treated with an IL-6 antibody to assess the impact on mucus metaplasia. $A d a^{-/-}$mice displaying pulmonary phenotypes at postnatal day 18 were subjected to treatment of anti-IL6 or Isotype antibody and were sacrificed at postnatal day 21 to assess mucin production (Fig 3.17a). Tissue sections from these mice were stained with PAS, and morphometry using Image-Pro allowed for quantification of mucous cell metaplasia. As previously reported, Ada $^{-/-}$mice displayed an increase in mucinproducing cells in the bronchial airways (119) (Fig 3.17b, middle panel). However, treatment with anti-IL-6 antibody led to the reduction of mucin 


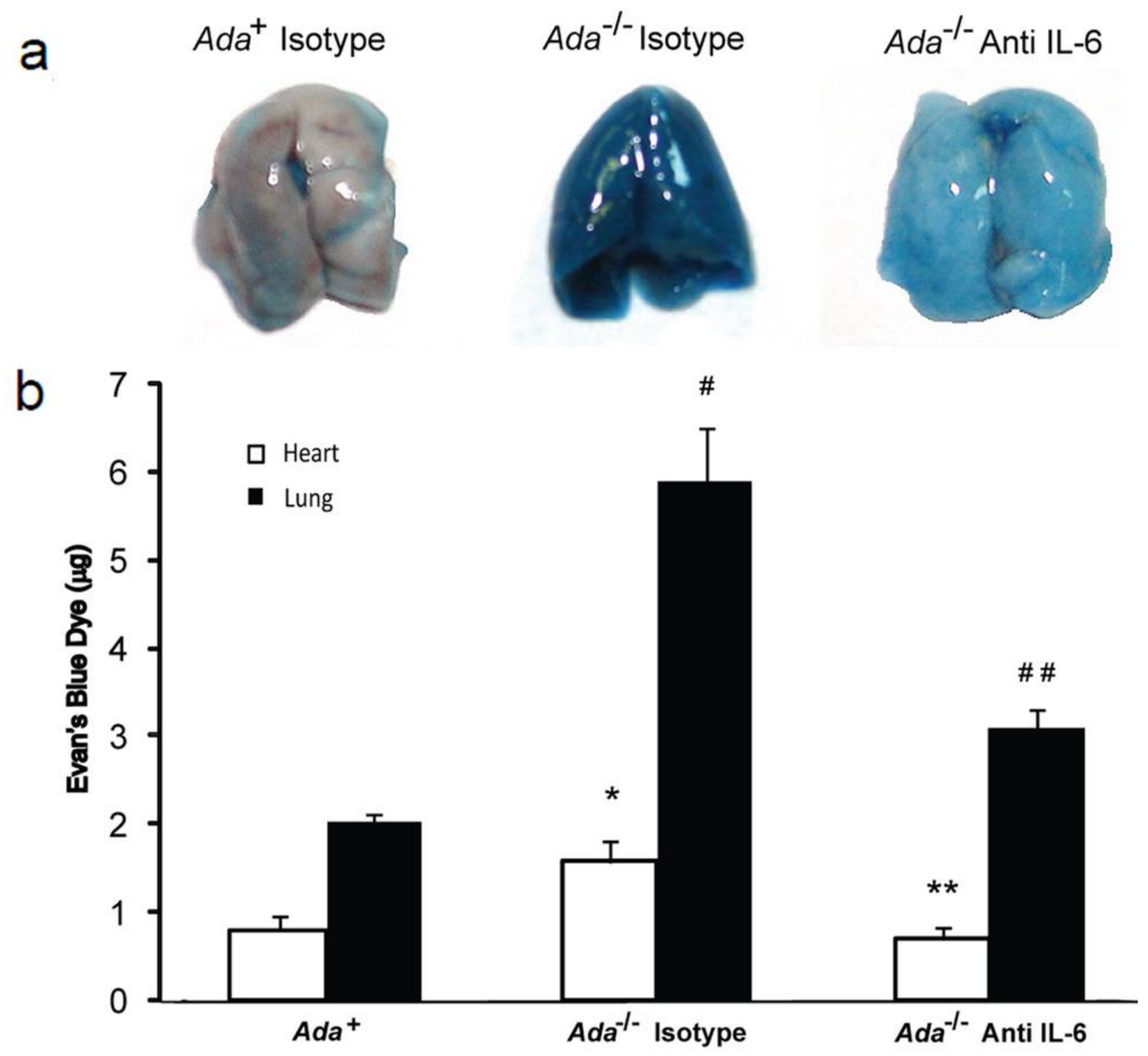

Figure 3.16

Vascular permeability following neutralization of IL-6 in ADA-deficient mice.

Vascular permeability analysis in $\mathrm{Ada}^{-/-}$and $\mathrm{Ada}^{+}$mice were treated with an IL-6 neutralizing antibody. At postnatal day 43 , mice were subjected to intraperitoneal injections with Evans Blue Dye and the heart and lungs were collected $4 \mathrm{hrs}$ after injection. (a) Representative images of lungs. (b) Evans blue dye concentrations were quantified in the heart and lungs. Data are presented as mean dye content in micrograms per gram of lung tissue \pm SEM, $n \geq 4 .\left(^{*}, p \leq 0.05\right.$ $A d a^{+}$vs. $A d a^{-/-}$and \#, p $\leq 0.05 A_{d a a^{-/}}$vs. Ada ${ }^{-/-}+$IL-6 Antibody). 
production in the bronchial airways of $\mathrm{Ada}^{-/}$mice (Fig 3.17b). Quantification of the PAS-positive staining revealed significant differences in mucus levels following IL-6 neutralization in comparison isotype control antibody (Fig 3.17c) Real-time PCR was used to quantify the RNA transcripts of major mucin genes responsible for mucus metaplasia, such as Muc5ac, Muc5ab, and the mucous metaplasia marker calcium-activated chloride channel 3 (CLCA-3). Although no significant differences were observed in Muc5ac and Muc5ab (data not shown), anti-IL-6 treated Ada $^{-/}$mice revealed a significant reduction in CLCA-3 (Fig 3.17d). This suggests that IL-6 regulates mucin secretion in the $\mathrm{Ada}^{-/-}$mice.

\section{STAT-3 ACTIVATION IN EPITHELIAL CELLS IN ADA-DEFICIENT MICE FOLLOWING NEUTRALIZATION OF IL-6}

STAT-3 is known to be the major effector of IL-6. To examine the contribution of IL-6 in STAT-3 activation in Ada ${ }^{-/-}$mice, western blot analysis was performed. Results revealed that $A d a^{-/-}$mice exhibit increased levels of phospho-STAT-3, consistent with previous observations (Fig 3.3); however, Ada-

1- mice treated with IL-6 neutralizing antibodies demonstrated a substantial reduction of phospho-STAT-3 activation (Fig 3.18a, c). As a confirmation of potential IL-6 cellular targets, phospho-STAT-3 immunolocalization revealed nuclear phospho-STAT-3 positive staining on bronchial airway epithelial cells and alveolar airway epithelial cells in isotype treated $\mathrm{Ada}^{-/-}$mice. However, the observed increased phospho-STAT-3 nuclear staining was prevented in anti-IL-6 treated $\mathrm{Ada}^{-/-}$mice (Fig 3.18c, right panel). These results indicate that the 


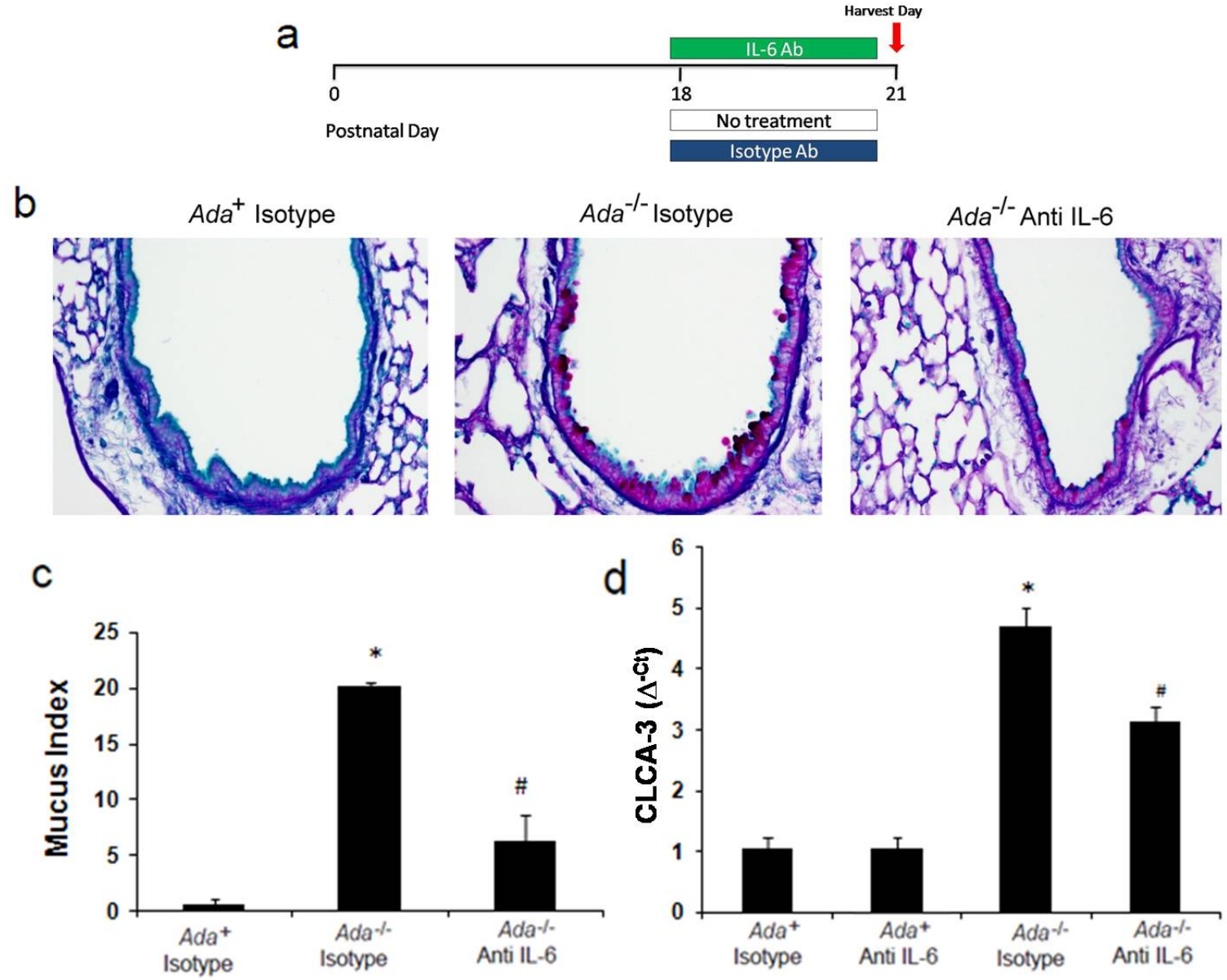

Figure 3.17

Mucus Production following neutralization of IL-6 in ADA-deficient mice.

Mucus production in $A d{ }^{-/}$mice treated with the anti-IL-6 antibody. Lung sections from postnatal day 21 mice were stained with PAS to detect mucus deposition (pink stain). (a) Schematic diagram illustrating experimental design of a single dose of anti-IL-6 via subcutaneous injection $(30 \mathrm{mg} / \mathrm{kg}$ ) at postnatal day 18, where pathological symptoms (lung inflammation and airway enlargement) are displayed. (b) Examination of mucus production revealed a reduction of bronchial airways in the lungs of $A d a^{-/-}$mice treated with the IL-6 neutralizing antibody. (c) Mean mucus index showing the extent of mucus production determined by quantifying the amount of PAS-stained material using Image-Pro analysis. (d) Transcript levels of CLCA-3 were measured in whole-lung RNA extracts from postnatal day 21 mice using quantitative RT-PCR. Transcripts were measured in parallel with $18 \mathrm{~S}$ rRNA and values are presented as mean normalized transcript levels $\left(\Delta^{-\mathrm{ct}}\right) \pm \pm$ SEM, $\mathrm{n} \geq 4$. $\left(^{*}, \mathrm{p} \leq 0.05 \mathrm{Ada}^{+}\right.$vs. Ada ${ }^{-/-}$and \#, $\mathrm{p} \leq 0.05$ Ada $^{-/-}$vs. Ada ${ }^{-/-}+$IL-6 Antibody). 


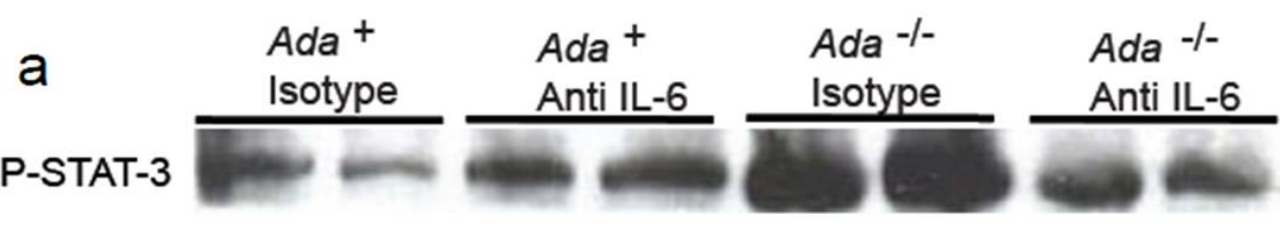

STAT-3
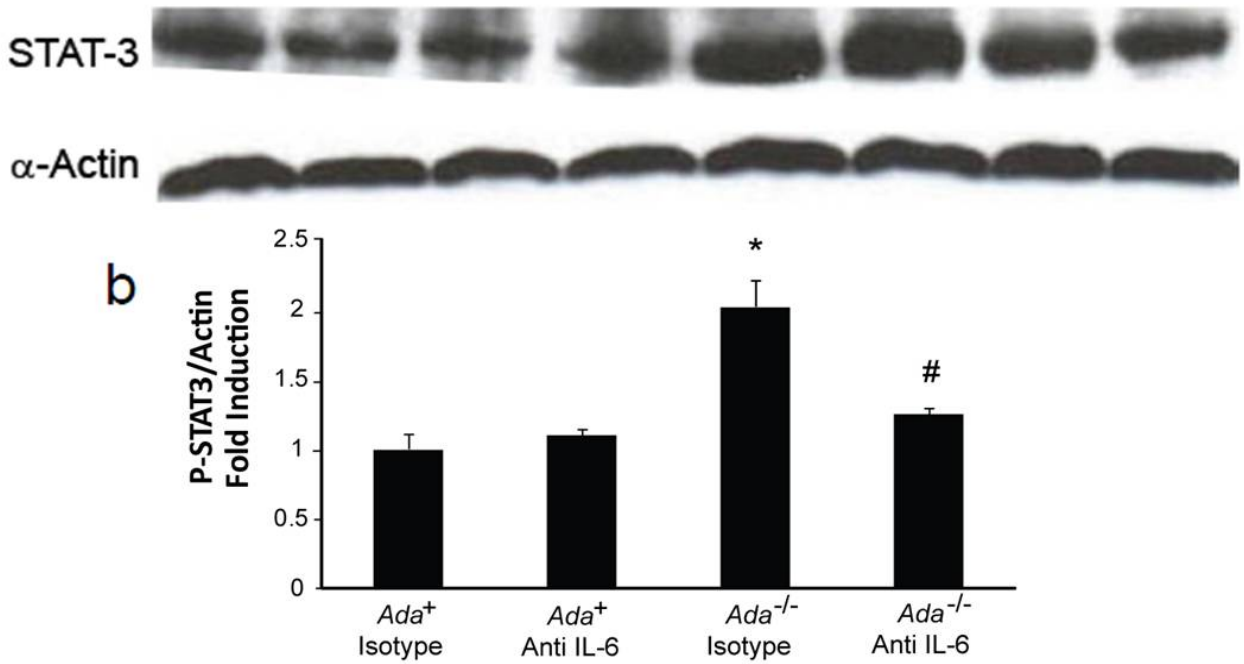

C $\mathrm{Ada}^{+}$Isotype
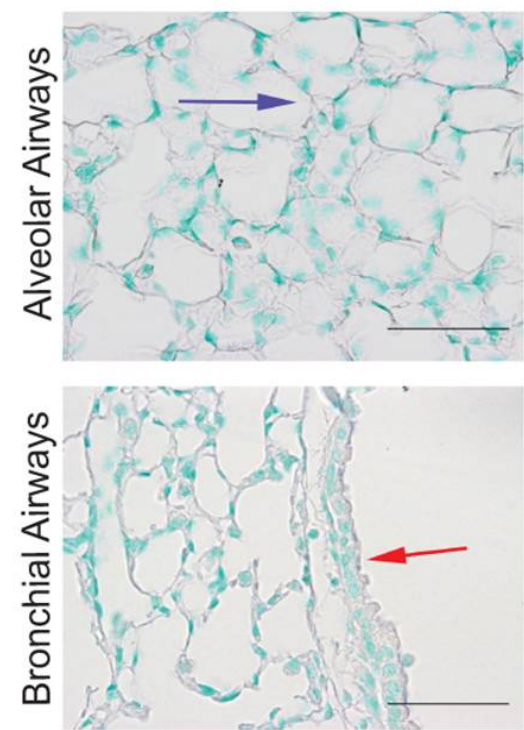

$\mathrm{Ada}^{-/-}$Isotype
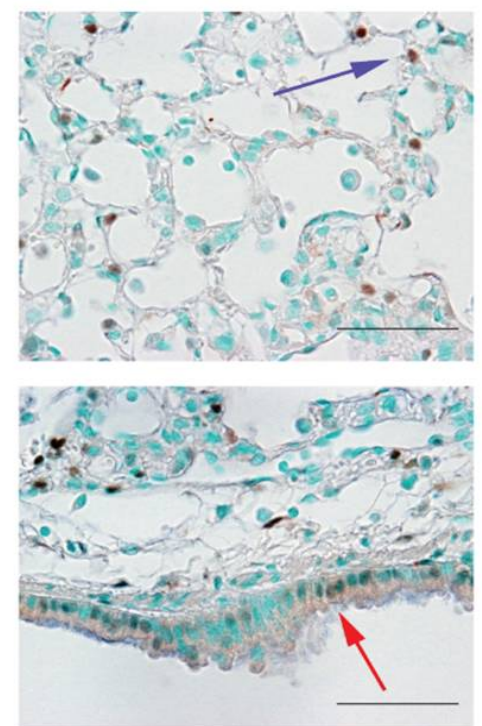

Ada ${ }^{-/-}$Anti IL-6
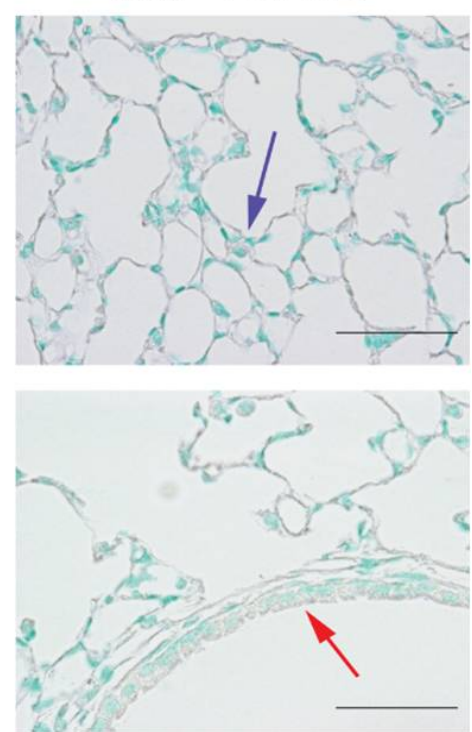

\section{Figure 3.18}

\section{STAT-3 activation following genetic removal of IL-6 in ADA-deficient mice.}

Phospho-STAT-3 expression assessed using western blot analysis (a), ImageJ analysis (b), and immunohistochemistry (c). STAT- 3 and $\alpha$-actin were used as loading controls. Values are presented as the percentage of $\alpha$-actin $\pm S E M, n \geq 4$. $\left({ }^{*}, \mathrm{p} \leq 0.05 \mathrm{Ada}^{+}\right.$vs. Ada ${ }^{-/-}$and \#, p $\leq 0.05 \mathrm{Ada}^{-/-}$vs. Ada ${ }^{-/-}+\mathrm{IL}-6$ Antibody). Lung sections were stained with an antibody specific for phospho-STAT-3, alveolar epithelial cells (blue arrows), bronchial epithelial cells (red arrows). Images are representative of 8 animals from each group. Scale bars: $100 \mu \mathrm{m}$. 
increased levels of phospho-STAT-3 activation displayed in the $\mathrm{Ada}^{-/-}$mice can be prevented by neutralizing IL-6. Thus, these observations suggest that IL-6 is the main activator of phospho-STAT-3 in the lungs of $\mathrm{Ada}^{-/-}$mice, and that blocking IL-6 mediated activation of STAT-3 may be an effective therapy for the treatment of pulmonary fibrosis.

\section{DISCUSSION}

Adenosine is a potent extracellular signaling molecule produced by various cells during normal metabolism (41) or as a result of cellular stress and damage (57-60). Adenosine is able to activate either anti-inflammatory (tissue protective) or pro-inflammatory (tissue destructive) pathways $(62-64,67)$ depending on the type and duration of injury, the amount of adenosine produced, and the alteration in the expression of adenosine receptors. Ada ${ }^{-/-}$mice display effects of chronic adenosine elevations, such as inflammation, alveolar air-space destruction, mucus metaplasia, and fibrosis (63). Pharmacological studies demonstrated that the $A_{2 B} R$ regulates the expression of key cytokines, chemokines, and proteases in conjunction with the adenosine-dependent phenotypes. In particular, inhibition of the $A_{2 B} R$ led to reduction in IL-6 production (79) implicating a role for this profibrotic cytokine in the pathological conditions of inflammation and fibrosis in this model. To confirm a role of IL-6 in the development of pulmonary phenotype, genetic studies involving Ada/IL- $\sigma^{/-}$ mice were analyzed. In addition, $A d a^{-/-}$mice were treated systemically with IL-6 neutralizing antibodies. Thus, the role of IL-6 in this adenosine-mediated 
pulmonary injury model was determined via complementary or confirmatory assays through neutralization and genetic studies.

Our results demonstrated that genetic removal of IL-6 in $A d a^{-/-}$mice led to reduced features of chronic lung disease, including inflammation, airspace enlargement, and fibrosis. Furthermore, the neutralization of IL-6 corroborated these observations suggesting blocking IL-6 signaling may have clinical significance. To pursue the mechanism by which IL-6 contributes to these fibrotic features, analysis of phospho-STAT-3 expression displayed elevations in this pathological condition. This implies that IL-6 is signaling through STAT-3 activation as a mechanism of action to contribute to the development and progression of pulmonary inflammation and fibrosis.

My results also indicated a significant decrease in airway inflammation in Ada ${ }^{-/}$mice after the genetic removal of IL-6 or the administration of IL-6 neutralizing antibodies, with significant reduction of macrophages, neutrophils, lymphocytes, and eosinophils. These findings confirm that IL-6 serves as an important mediator for pulmonary inflammation in models of chronic lung disease. Neutralization of IL-6 in Ada ${ }^{-/}$mice led to significant reduction of MCP1 and MIP-2 could account for the decreased macrophage recruitment, whereas reduction in OPN and CXCL-1 could account for the decreased neutrophilic infiltration. Previous reports demonstrate that activation of STAT-3 by IL-6 regulates neutrophil trafficking in acute inflammation (139). Influx of neutrophils by OPN has been associated with air-space enlargement and remodeling via regulation of MMPs (140). In addition, neutrophil accumulation leads to shedding 
of slL-6R promoting IL-6 trans-signaling events. These events consist of decreasing CXCL1, CXCL8, and Fructalkine (141); conversely driving the expression of CXCL5 (ENA-78), CXCL6 (GCP2), and MCP-1 (141). This immunological shift leads to neutrophil clearance and macrophage accumulation. Thus IL-6 trans-signaling is thought to be responsible for the transition from acute to chronic inflammation (142). Regarding the role of IL-6 in macrophages, IL-6 is able to promote differentiation-inducing signals when STAT-3 is suppressed leading to M1 macrophage differentiation, whereas M2 macrophage polarization occurs upon STAT-3 activation (143). This M2 lineage influences Th2-mediated responses and produces mediators that contribute to disease progression and fibrosis (144). Thus, this study demonstrates that during chronic pulmonary phenotypes, Ada $^{-/}$mice exhibit increased activation of STAT-3, which is involved in M2 differentiation and successively in Th-2 mediated responses. Hence, neutralizing IL-6 in this model leads to halted STAT-3 activation; this could consequentially lead decreased M2 differentiation and diminished Th2 responses. Furthermore, our results indicate that neutralization of IL-6 in $A d a^{-/}$ mice results in reduced expression of OPN, CXCL-1, MCP-1, and MIP-2 (Fig 3.13) thereby resulting in decreased neutrophil and macrophage infiltration, and preventing the production of mediators that contribute to the chronic state of this model.

Regarding lymphocytes, STAT-3 activation via IL-6 trans-signaling directs T cell migration in acute inflammation by up-regulating expression of CCL4 (MIP$1 \beta)$ and CCL5 (Rantes). IL-6 mediated events in T cells include polarization, 
adhesion, and anti-apoptotic effects contributing to the development of chronic inflammation (145). My findings underline the complex role of IL-6 in inflammation, namely that IL-6 is involved in neutrophil mobilization and activation (141), while at the same time is able to regulate the transition from acute to chronic inflammation by increasing the mononuclear-cell infiltrate and favoring the neutrophilic infiltrate clearance (142). Thus, it is conceivable that an imbalance in this transition from innate to acquired immunity leads to a distorted immunological regulation that results in disease progression. The factors that govern this inappropriate regulation remain unclear.

Additionally, IL-17 was another important cytokine that I found to be reduced by IL-6 neutralization. IL-17-producing T helper cells (Th17 cells) are functionally important in host defense and its aberrant regulation is involved in the pathogenesis of multiple inflammatory and autoimmune disorders (102). The differentiation of Th17 cells is controlled by TGF- $\beta$ and IL-6, which initiates the lineage commitment by inhibiting Foxp3 and activating the transcription factors STAT-3 and RORyt (146). While IL-6 is important for the generation of Th17 cells, IL-23 is important for their maintenance, expansion, and survival. IL-6deficient mice do not develop Th17 differentiation, but TGF- $\beta$ and IL-21 are able to activate an alternative pathway to induce Th17 cells in the absence of IL-6 (147). In $\mathrm{Ada}^{-/-}$mice, IL-17 is upregulated suggesting that ADA deficiency is associated with Th17 autoimmunity. However, $A d a^{-/}$mice treated with IL-6 neutralizing antibodies had decreased IL-17 mRNA levels (Fig 3.13d). 
Downregulation of IL-17 via neutralization of IL-6 suggests that the pathogenic Th17 cells can be prevented by inhibiting the initial lineage commitment.

In addition to reduced inflammation, IL-6 neutralization in $A d a^{-/-}$mice led to decreased alveolar destruction. Analysis of important alveolar destruction mediators after IL-6 neutralization revealed that IL-6 is involved in the regulation of proteases and anti-proteases, such as MMP-9 and TIMP-1 (Fig 3.15). The regulation of matrix metalloproteases by IL-6 has been reported previously (148). Furthermore, IL-6 is able to induce TIMP-1 expression in synovial fibroblasts via trans-signaling (149). This suggests that IL-6 exerts its contribution to alveolar destruction in this model via the regulation of the expression of important cytokines, chemokines, and proteases.

IL-6 neutralization in Ada $^{-/-}$mice also resulted in rescued pulmonary vascular permeability (Fig 3.16). In addition to the role of IL-6 mediating the inflammatory response, this preservation in vascular permeability may account in part for the decreased inflammatory cells (Fig 3.11). In hypoxia-associated vascular leakage, genetic removal or pharmacological blockage of the $A_{2 B} R$ has been shown to disrupt vascular permeability (150). Furthermore, Ada $^{-/-}$mice show enhanced pulmonary vascular leakage, which is further enhanced in $A D A / A_{2 B} R$ double knock out mice (129). This could be explained by the $A_{2 B} R$ induced activation of PKA, which phosphorylates vasodilator-stimulated phosphoprotein (VASP) leading to barrier function disruption (151). Hence, both models of hypoxia and ADA deficiency demonstrate that adenosine signaling regulates pulmonary vascular permeability. Moreover, IL-6 is able to contribute 
to gastrointestinal vascular leakage via downregulation of tight junction proteins, such as ZO-1 (138). Hence, IL-6 neutralization may preserve pulmonary vasculature in $A d a^{-/}$mice by preventing downregulation of tight junction protein metabolism.

Another prominent feature of $A d a^{-/-}$mice is increased mucus production. In chronic inflammatory diseases, such as asthma, mucus production from lung epithelium leads to obstruction of the airways culminating in increased airflow resistance $(79,119)$. Homeostatic mechanisms regulates mucus production and airway clearance in order to maintain proper lung function (152). Perturbation of these mechanisms leads to mucus hypersecretion and eventually lung dysfunction. In response to fungal allergens, it has been shown that IL-6 is necessary for mucin overproduction (153). It has been suggested that IL-6 modulates mucus production as a consequence of regulating IL-13 expression (153). In human lung epithelial cell lines, IL-6 is able to upregulate the mucin genes Muc5ac and Muc5b (154). Although Muc5ac and Muc5b levels are increased in $A d a^{-/}$mice, no significant changes were observed following IL-6 neutralization. However, downregulation of CLCA-3 was observed in anti-IL-6 treated $\mathrm{Ada}^{-/}$mice (Fig 3.17d). This suggests that IL-6 regulates mucous cell metaplasia by modulating levels of CLCA-3 in the $\mathrm{Ada}^{-/-}$mice.

The results in this chapter demonstrate that IL-6 is produced in an adenosine-dependent manner largely from alveolar macrophages. This appears to be sufficient to induce STAT-3 activation on alveolar epithelial cells. Functionally, IL-6 has important roles in $A d a^{-/}$mice. Genetic removal or 
neutralization of IL-6 in this model led to improved lung phenotypes in particular reduced inflammation, decreased airway enlargement, and diminished fibrosis. Although these results demonstrate that IL-6 contributes to the pulmonary phenotypes in the $A d a^{-/}$mice, analysis of IL-6 in pulmonary pathologies needs to be further extended to more conventional models in order to promote the development of novel IL-6 therapies. 


\section{CHAPTER FOUR \\ THE ROLE OF IL-6 IN}

BLEOMYCIN-INDUCED

PULMONARY FIBROSIS 


\section{INTRODUCTION}

In the previous chapter, it was demonstrated that the genetic removal of IL-6 and the IL-6 neutralization in $A d a^{-/-}$mice resulted in decreased lung inflammation and fibrosis in association with decreased STAT-3 activation in alveolar airway epithelial cells. This implies that STAT-3 activation via IL-6 signaling is a mechanism of action that contributes to the development and progression of pulmonary inflammation and fibrosis in $\mathrm{Ada}^{-/-}$mice.

In this chapter, to further confirm the contribution of IL-6 to inflammation and fibrosis in chronic lung diseases, I will utilize the bleomycin model, which is the most commonly used model to study pulmonary fibrosis. As with $\mathrm{Ada}^{-/}$mice, I will use both a genetic and pharmacological approach to investigate the role of IL-6 in this model. The neutralization and genetic studies from both models will serve as confirmatory and/or complementary assays to evaluate the role of IL-6 in adenosine-dependent pulmonary injury and fibrosis.

\section{INTRAPERITONEAL BLEOMYCIN-INDUCED PULMONARY FIBROSIS}

The most common method to deliver bleomycin is intra-tracheal (i.t.) administration (155). The i.t. administration of bleomycin is a model of acute injury followed by resolvable fibrosis, which is inconsistent with human disease. This method is performed on 10-week old female mice exposed to a single i.t. dose of $2.5 \mathrm{U} / \mathrm{kg}$ bleomycin. I.T bleomycin administration directly into the respiratory tract results in extensive apoptosis of airway epithelial cells. This acute damage results in an injury phase characterize by the influx of 
inflammatory cells by Day 7 post-challenge. However, by Day 21 post-challenge, inflammation has resolved and fibrosis is at its maximum. This method results in a non-homogenous delivery of bleomycin that results in patchy fibrosis. Furthermore, due to the single delivery, the pulmonary fibrosis is temporal and resolves by Day 48 post-challenge. This model does not represent what happens in the lungs of IPF patients. In contrast, repeated intra-peritoneal (i.p.) injections of bleomycin are associated with diminished acute injury and progressive and irreversible pulmonary fibrosis. Thus, the i.p. bleomycin model resembles to a greater extent what is observed in IPF. For this reason, we will use the i.p. bleomycin model in our analysis of the contribution of IL-6 to pulmonary pathology.

\section{GENETIC REMOVAL OF IL-6 IN THE BLEOMYCIN MODEL}

C57BL/6J male mice at four to five weeks of age were challenge with bleomycin $(0.035 \mathrm{U} / \mathrm{g})$ via i.p. injection. Established models revealed that male mice exhibit improved survival rates compared to females under the intraperitoneal injections (128). Therefore, only male mice were used in our i.p. model. To further understand the role of IL-6 in the development of pulmonary fibrosis, wild type mice and IL-6 knockout mice $\left(I L-\sigma^{-/}\right)$were challenged with bleomycin via i.p. administration. Mice were injected with bleomycin at Day 1, 4, $8,11,15,18,22$, and 25 . These mice were sacrificed at Day 33 post-initial challenge. Control groups were injected with phosphate buffered saline (PBS) instead of bleomycin (Fig 4.1). These studies were designed to further expand 
our overall understanding of the contribution of IL-6 in experimental pulmonary fibrosis.

\section{IL-6 NEUTRALIZATION IN THE BLEOMYCIN MODEL}

To confirm the role of IL-6 in the development of pulmonary fibrosis, neutralization of IL-6 was performed in mice subjected to the i.p. bleomycin model. Control groups were injected with PBS instead of bleomycin. To assess the therapeutic potential of IL-6 neutralization in this model, wild type mice were treated subcutaneously with anti-IL-6 antibodies $(30 \mathrm{mg} / \mathrm{kg})$. The anti-IL-6 injections were performed on Day 15, 18, 22, and 25. These animals were sacrificed at Day 33 after the initial injection. It is important to note that pulmonary fibrosis is established by this stage in the protocol (Fig 4.1). Thus, these studies were designed to assess the ability of IL-6 neutralization to halt and resolve active fibrosis.

\section{SPECIFIC QUESTIONS AND EXPERIMENTAL RATIONALE}

The implementation of a second model to study the role of IL-6 in pulmonary phenotypes results from the complementation of these two models. $A_{d a}{ }^{-/}$mice are an adenosine-dependent model of lung injury. Thus, the pulmonary phenotype displayed by $A d a^{-/-}$mice can be prevented and reversed by lowering adenosine levels with ADA enzyme replacement therapy (119). These findings suggest that elevations of adenosine regulate pathways that promote the pathogenesis of chronic lung diseases. Hence, the ADA-deficient mouse is a 
valuable tool to study the in vivo physiology of increased adenosine levels whereby establishing an adenosine-dependent model for chronic lung diseases. However, the elevation of the ADA substrates, namely adenosine and 2'deoxyadenosine, in Ada $^{-/-}$mice results in a severe immunodeficiency similar to ADA deficiency in humans (64). In addition, the phosphorylation of 2'deoxyadenosine leads to high levels of dATP, which induces apoptosis in lymphocytes (64). The bleomycin mice model will compensate for the observed lymphopenia in the $\mathrm{Ada}^{-/}$mice. The bleomycin model is a useful as a tool for the study pulmonary fibrosis (40). Bleomycin is currently used as a chemotherapeutic agent, but side effects include impaired lung function and pulmonary toxicity resulting in pulmonary fibrosis (156). Thus, this side-effect increases its relevance in studying pulmonary fibrosis. However, IPF, as the name implies, results from unknown causes. Therefore, the bleomycin model is one way of developing fibrosis, but not the only way. This is corroborated with the vast antifibrotic agents that are able to reduce fibrosis in the bleomycin model, but are not successfully translated into clinical relevance in humans (40). Thus, studying the development of fibrosis in the $A d a^{-/}$mice is a complementary way to identify overlapping mechanisms underlying the development of pulmonary fibrosis in response to heterogeneous stimuli.

Similar to the $A d a^{-/}$mice, the bleomycin model shows elevations of adenosine and IL-6. This suggests that IL-6 plays a significant role in disease progression in these models. As was shown in the previous chapter, the role of IL-6 in adenosine-mediated pulmonary injury was determined via complementary 
and confirmatory assays through genetic and neutralization studies. Thus, the neutralization and genetic studies from both models serve as confirmatory and complementary assays to evaluate the role of IL- 6 in these models of adenosinedependent pulmonary injury and fibrosis.

\section{RESULTS}

REDUCED PULMONARY INFLAMMATION, FIBROSIS AND STAT-3 ACTIVATION IN IL-6-DEFICIENT MICE EXPOSED TO BLEOMYCIN

\section{PULMONARY PATHOLOGY IN IL-6 KNOCKOUT MICE TREATED WITH BLEOMYCIN}

Adenosine is able to induce pro-fibrotic effects in the setting of prolonged elevations (79). Bleomycin-induced pulmonary fibrosis is associated with increased adenosine levels and attenuation of fibrosis is observed with adenosine-based therapies (79). To assess the role of IL-6 in the bleomycin

model, IL-6 knock out mice were subjected to i.p. exposure of bleomycin twice a week for four weeks. Examination of lung histopathology at the end of the protocol revealed that $I L-6^{--}$mice exposed to bleomycin had reduced pulmonary injury (Fig 4.2). These findings suggest that IL-6 contributes to the development and progression of pulmonary fibrosis in the bleomycin model. 


\section{Genetic Removal and Neutralization of IL-6 in the Bleomycin Model}

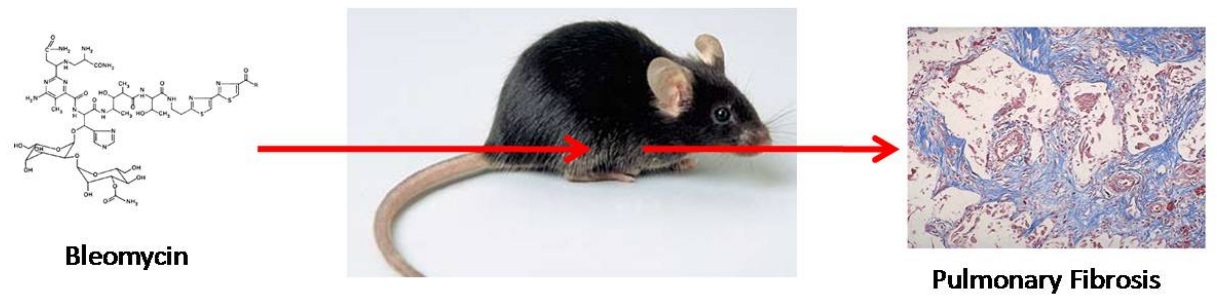

- Genetic Studies

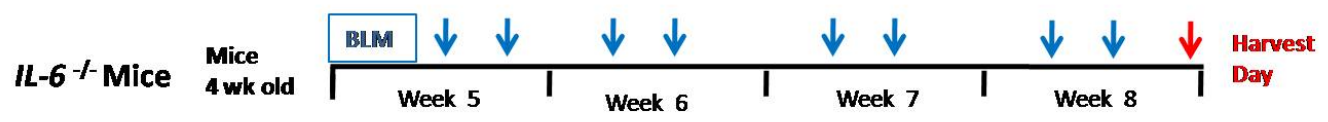

\section{- Neutralization Studies}

WT Mice

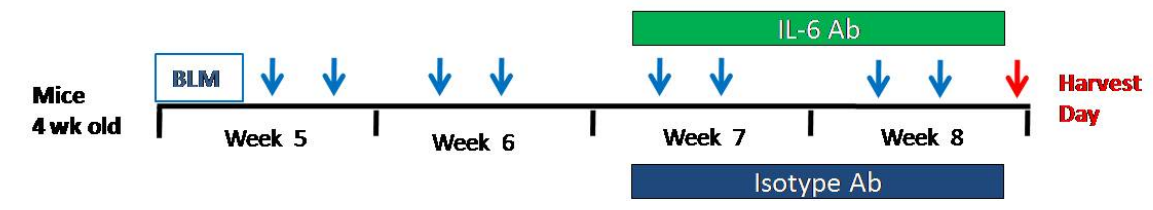

Figure 4.1

\section{Schematic Diagram of Methodology Employed in the Bleomycin Model.}

To determine the role of IL-6 in the bleomycin model two approaches were utilized. The genetic studies consisted of treating C57BL/6J mice (WT) and IL-6 knock out male mice $\left(I L-6^{-1}\right)$ at four to five weeks of age with bleomycin $(0.035$ $\mathrm{U} / \mathrm{g}$ ) via intra-peritoneal injections. After the fifth week of age, bleomycin injections were performed at Day 1, 4, 8, 11, 15, 18, 22, and 25. PBS treated mice were used as a control group. For the neutralization studies, C57BL/6J mice exposed to bleomycin as described above were treated subcutaneously with the anti-IL-6 antibody ( $30 \mathrm{mg} / \mathrm{kg}$ ) on Day 15, 18, 22, and 25 . All of these animals were sacrificed at Day 33 after the initial injection. Isotype antibody treated mice were used as a control group. 


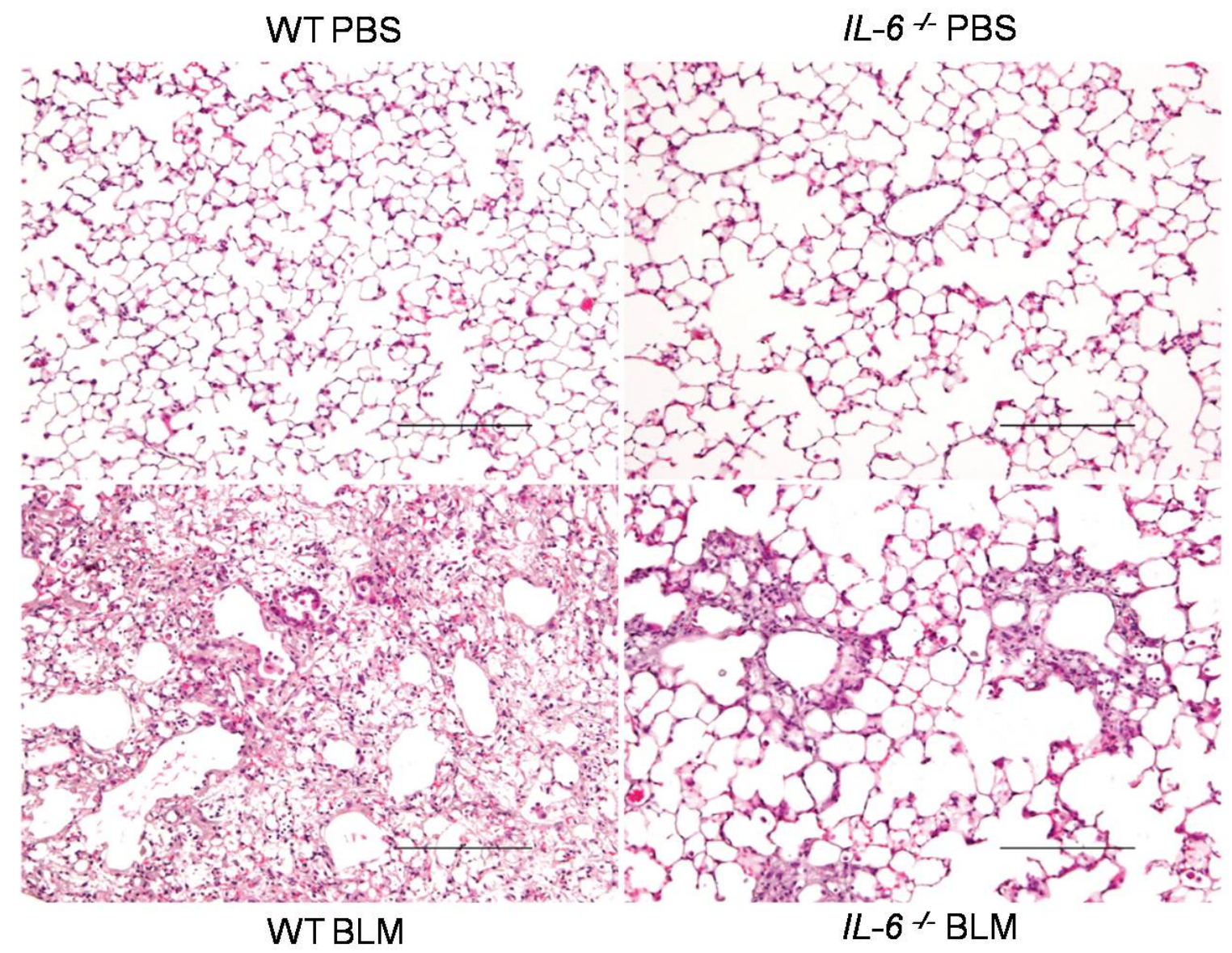

Figure 4.2

Pulmonary phenotypes following intraperitoneal bleomycin exposure in IL6 knock out mice.

To determine the role of IL-6 in the bleomycin model, the genetic studies consisted of treating C57BL/6J mice (WT) and IL-6 knock out male mice $\left(I L-6^{-/}\right)$ at four to five weeks of age with bleomycin via intraperitoneal injections for four weeks twice a week. Inflammatory and fibrotic characteristics were assessed from lung sections collected 33 days after initial bleomycin exposure. This figure displays representative histology of lungs from wild type mice treated with PBS (top left) and bleomycin (bottom left) and $I L-\sigma^{-\alpha}$ mice exposed to bleomycin (bottom right). Examination of lung histology through $\mathrm{H} \& E$ staining revealed that $I L-6^{-}$mice exposed to bleomycin displayed a reduction in lung inflammation and pulmonary fibrosis. Images are representative of 8 animals from each group. Scale bars: $200 \mu \mathrm{m}$. 


\section{PULMONARY INFLAMMATION IN IL-6 KNOCKOUT MICE TREATED WITH BLEOMYCIN}

To assess the role of IL-6 on inflammation in the bleomycin model, wild type and $I L-\sigma^{-}$mice were exposed with bleomycin. Pulmonary inflammation was reduced (Fig. 4.3a-C) in $I L-\sigma^{-}$mice subjected to bleomycin. Quantification of the level of inflammation in the BAL demonstrated a significant reduction in total cells (Fig 4.3a), and cellular differentials revealed reduction in macrophages (Fig 4.3b) and lymphocytes, eosinophils, and neutrophils (Fig 4.3c). This suggests that in the bleomycin model, IL-6 contributes to pulmonary inflammation.

\section{PULMONARY FIBROSIS IN IL-6 KNOCKOUT MICE EXPOSED TO BLEOMYCIN}

Although there are numerous animal models of pulmonary fibrosis, perhaps the most appropriate is the i.p. bleomycin model. This model develops similar features of IPF including intra-alveolar buds, mural incorporation of collagen, and obliteration of the alveolar space (140). To assess the role of IL-6 in bleomycin-induced fibrosis, $I L-\sigma^{/-}$mice were treated with bleomycin for four weeks twice a week. In comparison to bleomycin treated wild type mice, $I L-\sigma^{/-}$ mice exposed to bleomycin develop decreased collagen production (Fig 4.12a) and myofibroblast accumulation (Fig 4.12b). This reduction in fibrosis was

confirmed using Ashcroft scoring (Fig 4.12c). These findings suggest that IL-6 contributes to the development of pulmonary fibrosis in the intra-peritoneal model of bleomycin-induced injury. 

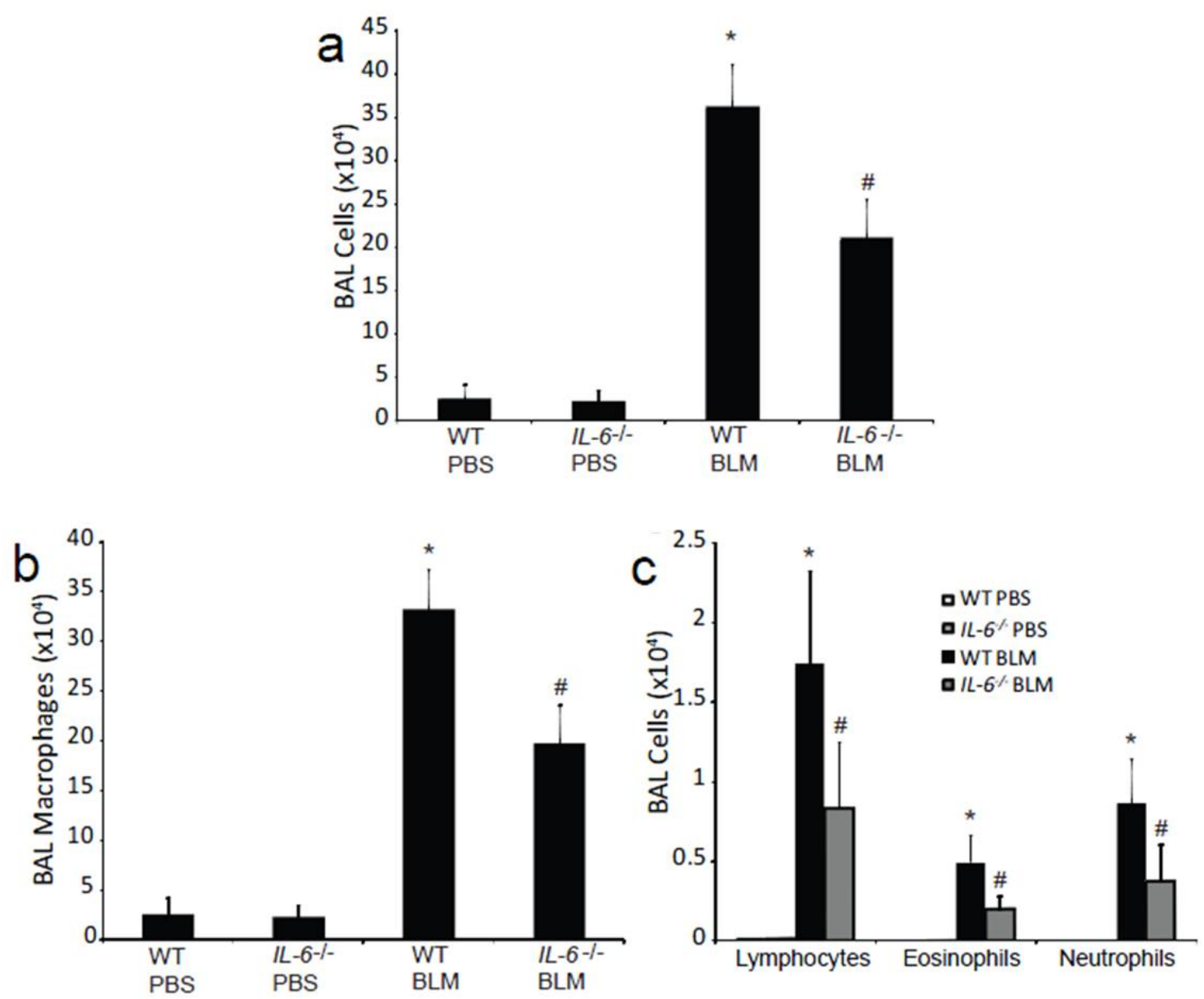

\section{Figure 4.3}

Pulmonary inflammation following intraperitoneal bleomycin exposure in IL-6 knock out mice.

Bronchoalveolar lavage (46) was performed on C57BL/6J mice (WT) and IL-6 knock out male mice $\left(I L-6^{-/}\right)$treated with bleomycin. (a) Total cells numbers in lavage fluid were counted using a hemocytometer. BAL cells were cytospun and stained with Diff-Quick, allowing for determination of macrophages (b) and lymphocytes, eosinophils, and neutrophils (c). Data are presented as mean cell counts \pm SEM, $n \geq 8$. ${ }^{*}, p \leq 0.05$ WT PBS vs. WT bleomycin and \#, $p \leq 0.05$ WT bleomycin vs. IL-6 ${ }^{-/-}$bleomycin). 

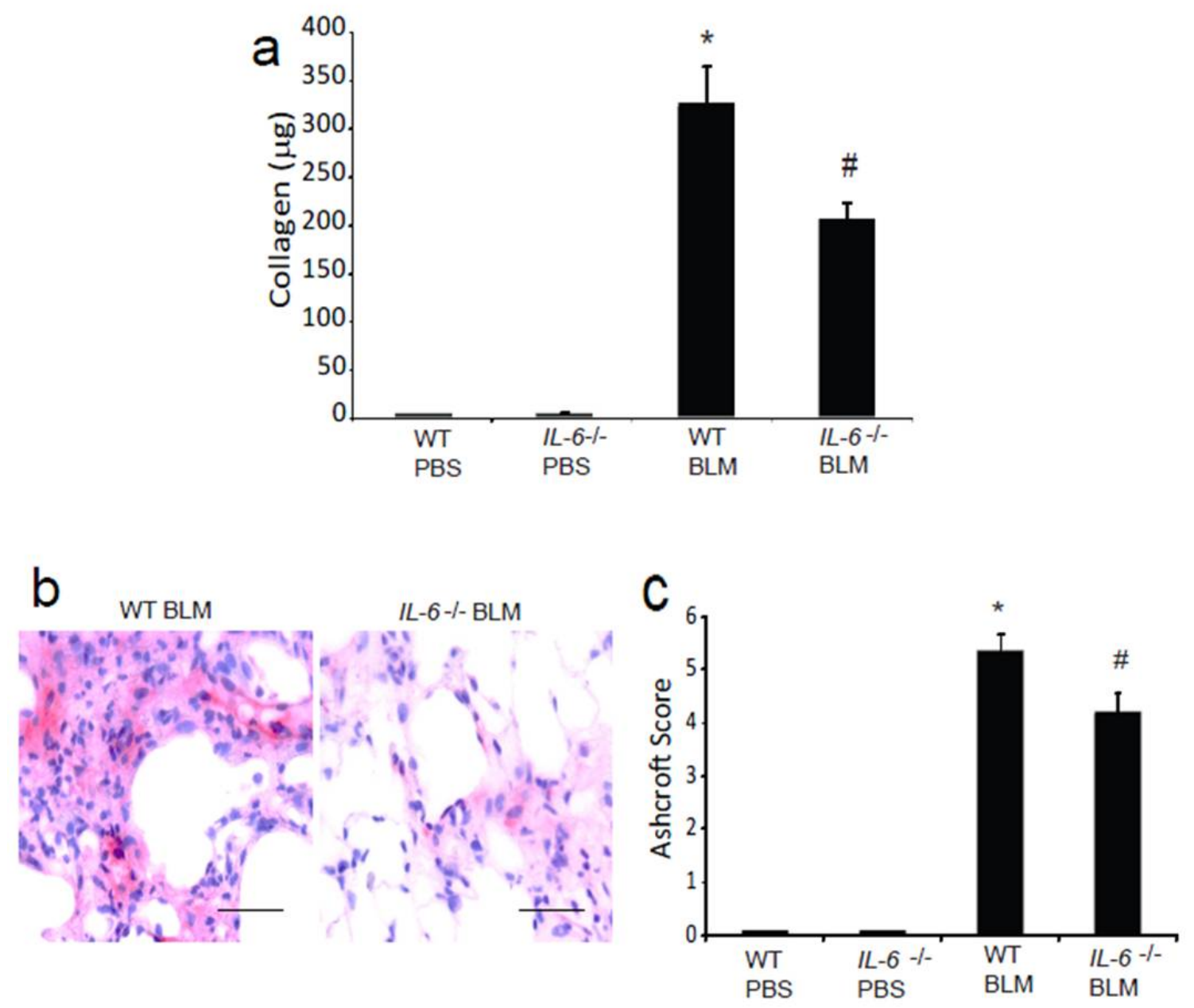

\section{Figure 4.4}

Pulmonary fibrosis following intraperitoneal bleomycin exposure in IL-6 knock out mice.

Fibrotic assessment was performed on C57BL/6J mice (WT) and IL-6 knock out male mice $\left(I L-6^{\prime-}\right)$ treated with bleomycin. (a) Soluble collagen protein levels were measured using Sircol Assay. Data presented as mean $\mu \mathrm{g}$ collagen $/ \mathrm{ml}$ BAL fluid \pm SEM, $n \geq 8$. ( ${ }^{*}, p \leq 0.05$ WT PBS vs. WT bleomycin and \#, $p \leq 0.05$ WT bleomycin vs. IL-6 antibody against for $\alpha$-SMA to visualize myofibroblast accumulation (pink stain). Images are representative of 6 animals from each group. Scale bars: $100 \mu \mathrm{m}$. (c) Ashcroft scores were used to determine the degree of fibrosis $\left({ }^{*}, p \leq 0.05\right.$ WT PBS vs. WT bleomycin and \#, $p \leq 0.05$ WT bleomycin vs. $I L-6^{-/-}$bleomycin, $n \geq 6$ ). 


\section{STAT-3 ACTIVATION IN AIRWAY EPITHELIAL CELLS IN MICE EXPOSED TO BLEOMYCIN}

Cellular activation of IL-6 involves phosphorylation of STAT-3 in target cells to induce different biological processes (90). To evaluate the cellular targets of IL-6 activation in the intra-peritoneal bleomycin model, western blot analysis was conducted on whole lung lysates at Day 33 of the protocol. Results revealed a significant reduction in STAT-3 activation in $I L-6^{-\alpha}$ mice exposed to bleomycin in comparison to wild type mice (Fig 4.5a). Immunolocalization of phospho-STAT-3 in wild type mice exposed to bleomycin revealed activation of alveolar epithelial cells next to fibrotic foci; presumably hyperplastic epithelial cells (Fig 4.5c). The increased STAT-3 activation in these cells was reduced in

$I L-6^{-/}$mice. These results are similar to the findings observed in $\mathrm{Ada}^{-/}$mice where the genetic removal or neutralization of IL-6 leads decreased pulmonary inflammation and fibrosis in association with decreased STAT-3 activation in alveolar epithelial cells (Chapter 3 ). These observations suggest that the IL-6 induced STAT-3 activation is associated with the regulation of pulmonary fibrosis.

REDUCED PULMONARY INFLAMMATION, FIBROSIS AND STAT-3 ACTIVATION FOLLOWING IL-6 NEUTRALIZATION IN MICE EXPOSED TO BLEOMYCIN

\section{PULMONARY PATHOLOGY IN BLEOMYCIN-EXPOSED MICE FOLLOWING IL-6 NEUTRALIZATION}

The bleomycin model is widely used in the assessment of antifibrotic compounds. Furthermore, IL-6 deficiency suggests a pro-fibrotic role of IL-6. Thus, to examine the therapeutic benefit of targeting IL-6 in the i.p. model of 

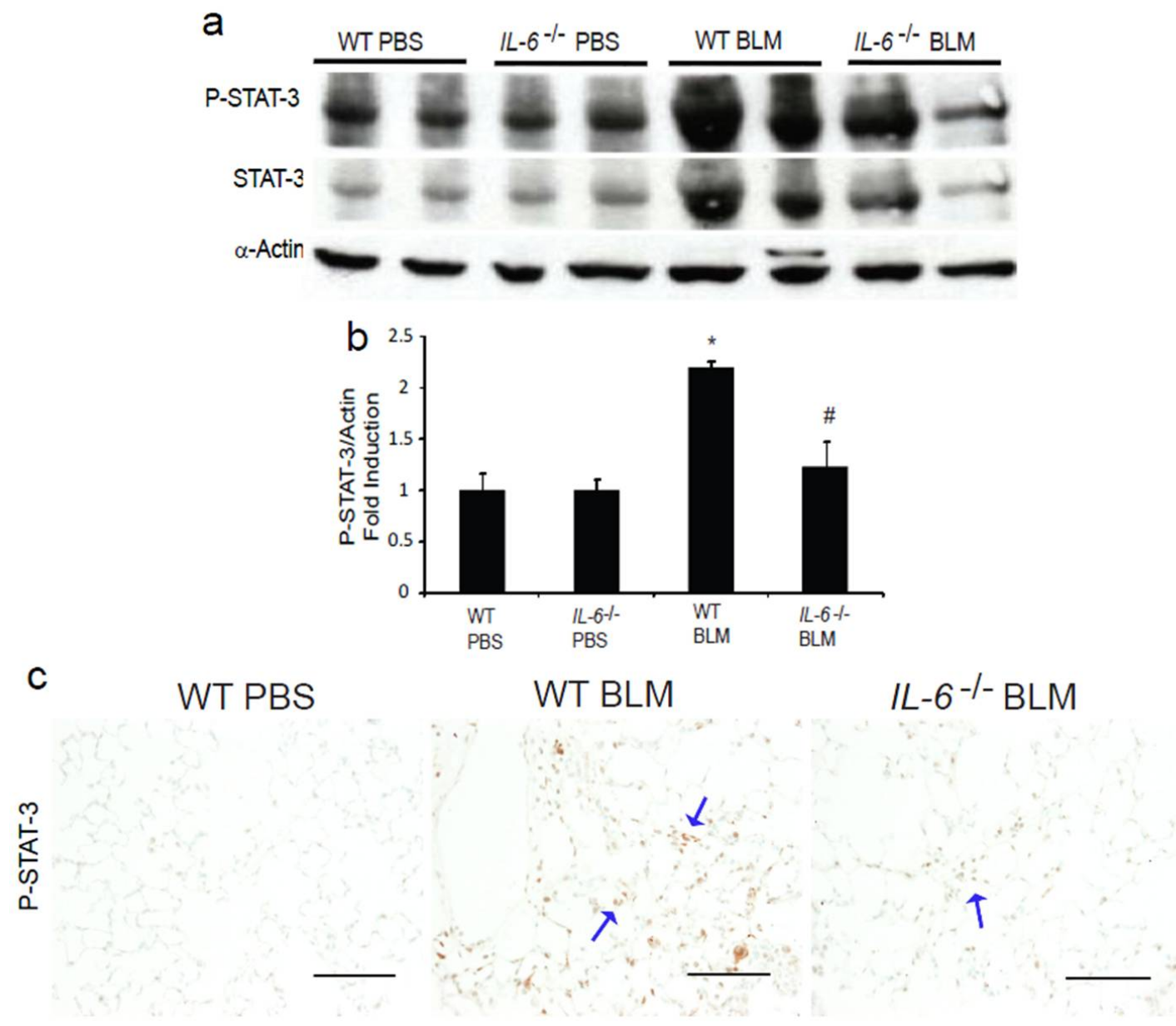

Figure 4.5

STAT-3 activation following genetic removal of IL- 6 in bleomycin treated mice.

Phospho-STAT-3 expression. (a) Western Blot analysis was performed using an antibody against phospho-STAT-3 in whole lungs lysates. STAT-3 and $\alpha$-actin were used as controls. (b) Phospho-STAT-3 band intensity was quantified using ImageJ analysis. Values are presented as the percentage of $\alpha$-actin $\pm \mathrm{SEM}$, $n \geq 4$. $\left({ }^{*}, p \leq 0.05\right.$ WT PBS vs. WT bleomycin and \#, $p \leq 0.05$ WT bleomycin vs. IL$6^{\%}$ bleomycin). (c) Immunolocalization of phospho-STAT-3 expression. Lung sections were stained with an antibody specific for phospho-STAT-3 on alveolar epithelial cells (blue arrows). Images are representative of 8 animals from each group. Scale bars: $100 \mu \mathrm{m}$. 
bleomycin injury, wild type mice exposed to bleomycin were treated with IL-6 neutralizing antibodies beginning at the stage after which fibrosis was established. Histopathologic features of lung injury were reduced in bleomycinexposed mice subjected to IL-6 neutralization (Fig 4.6). These findings suggest that IL-6 contributes to the development and progression of pulmonary fibrosis in the bleomycin model. Furthermore, neutralization of IL-6 in this model indicated that IL-6-blocking agents could have therapeutic benefits on pulmonary fibrosis.

\section{PULMONARY INFLAMMATION IN BLEOMYCIN-EXPOSED MICE FOLLOWING IL-6 NEUTRALIZATION}

To quantify the reduced pulmonary inflammation observed on histologic findings, BAL was performed and recovered inflammatory cells were counted using a hemocytometer. Results revealed characteristic increases in total inflammatory cells recovered from BAL fluid of wild type mice exposed to bleomycin versus PBS, but a significant reduction was observed with the use of IL-6 neutralizing antibodies (Fig 4.7). Decreased pulmonary inflammation was observed by the reduction in total inflammatory cells (Fig 4.7a), macrophages (Fig 4.7b), lymphocytes, eosinophils, and neutrophils (Fig 4.7c). These data suggest that IL-6 contributes to pulmonary inflammation in the bleomycin model. 

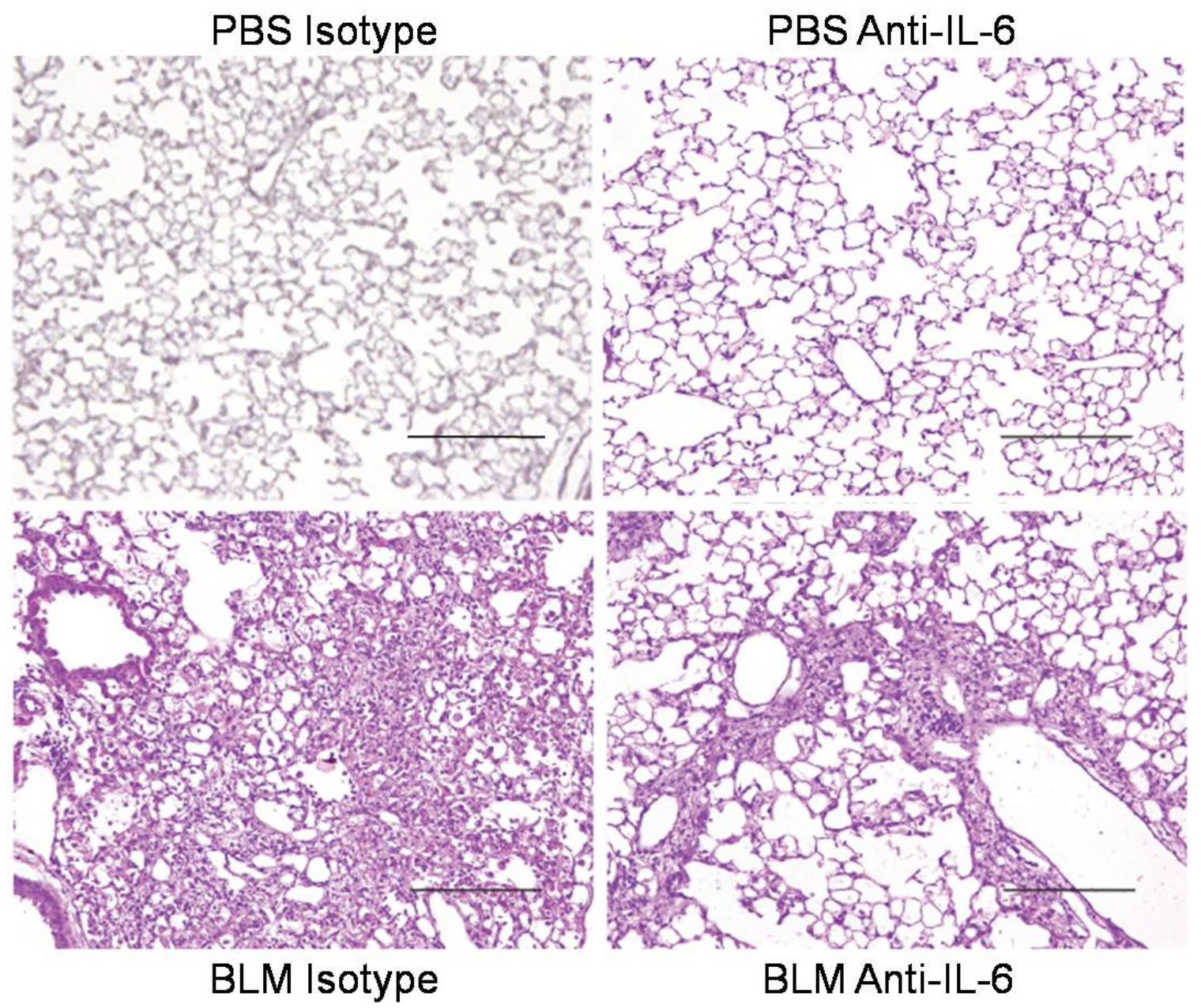

Figure 4.6

\section{Pulmonary phenotypes following intraperitoneal bleomycin exposure in mice treated with IL-6 neutralization antibodies.}

To determine the role of IL-6 in the bleomycin model, the neutralization studies consisted of treating C57BL/6J male mice at four to five weeks of age with bleomycin via intraperitoneal injections for four weeks twice a week. At the same time, these mice were treated subcutaneously with the anti-IL-6 antibody (30 $\mathrm{mg} / \mathrm{kg}$ ). The anti-IL-6 injections were performed on Day 15, 18, 22, and 25. Inflammatory and fibrotic characteristics were assessed from lung sections collected 33 days after initial bleomycin exposure. This figure displays representative histology of lungs from wild type mice treated with saline (top left) and bleomycin-exposed mice treated with isotype antibody (bottom left) or IL-6 antibody (bottom right). Examination of lung histology through H\&E staining revealed that neutralization of IL-6 displayed a reduction in lung inflammation and pulmonary fibrosis. Images are representative of 8 animals from each group. Scale bars: $200 \mu \mathrm{m}$. 

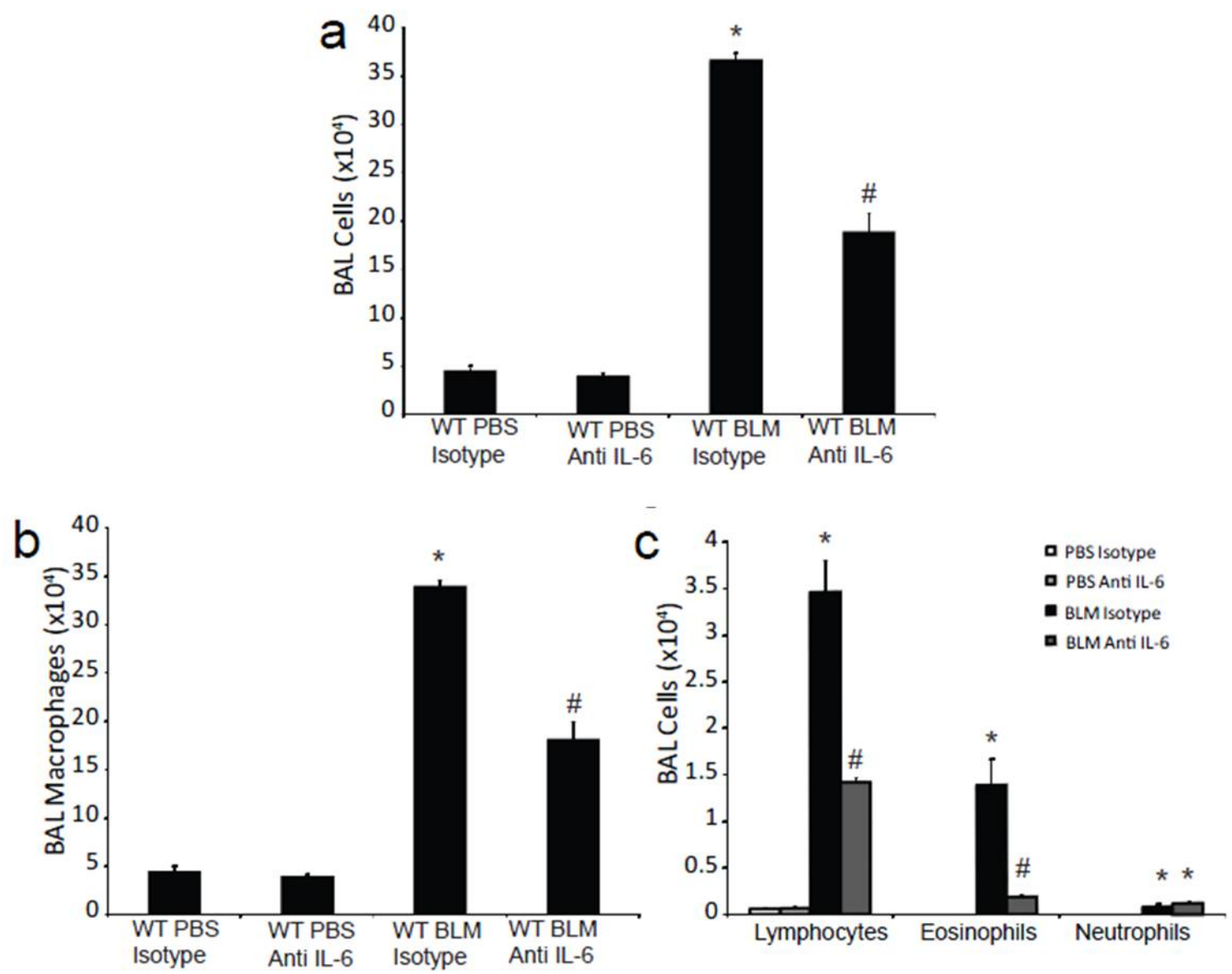

Figure 4.7

Pulmonary inflammation following intraperitoneal bleomycin exposure in mice treated with IL-6 neutralization antibodies.

Bronchoalveolar lavage (46) was performed on bleomycin-exposed mice treated with IL-6 neutralizing antibodies or isotype. Total cells numbers (a) and cellular differentials including macrophages (b) and lymphocytes, eosinophils, and neutrophils (c). Data are presented as mean cell counts \pm SEM, $n \geq 8 .\left({ }^{*}, p \leq 0.05\right.$ PBS + IL-6 Antibody vs. Bleomycin + Isotype and \#, $p \leq 0.05$ Bleomycin + Isotype vs. Bleomycin + IL-6 Antibody). 


\section{PULMONARY FIBROSIS IN BLEOMYCIN-EXPOSED MICE FOLLOWING IL-6 NEUTRALIZATION}

To assess the therapeutic benefit of targeting IL-6 on pulmonary fibrosis, bleomycin-exposed mice were treated with IL-6 neutralizing antibodies. Results revealed that IL-6 neutralization was associated with decreased pulmonary damage (Fig 4.6). In addition, wild type mice exposed to bleomycin displayed characteristics increases in collagen production and deposition and myofibroblast accumulation. However, treatment with IL-6 neutralizing antibodies at the stage of established fibrosis attenuated collagen production (Fig 4.8a), and myofibroblast accumulation (Fig 4.8c). These changes were associated with significant reduction in the quantification of pulmonary fibrosis as determined by Ashcroft scores (Fig 4.8d). Moreover, physiological analysis measuring arterial oxygen saturation demonstrated that IL-6 neutralization was associated with restored oxygen saturation in this model (Fig 4.8b). These observations suggest that IL-6 is a promising antifibrotic agent able to reduce pulmonary fibrosis and preserve clinically relevant properties such as arterial oxygen saturation.

\section{IL-6 DEPENDENT STAT-3 ACTIVATION IN EPITHELIAL CELLS IN BLEOMYCIN-EXPOSED MICE}

Similar findings regarding the increased activation of STAT-3 in this model

were determined by western blot analysis (Fig 4.9). Moreover, results demonstrated significant reduction in STAT-3 activation following anti-IL-6 treatment (Fig 4.9a, b). Identification of cellular targets via immunolocalization of phospho-STAT-3 demonstrated an increased activation in alveolar epithelial 


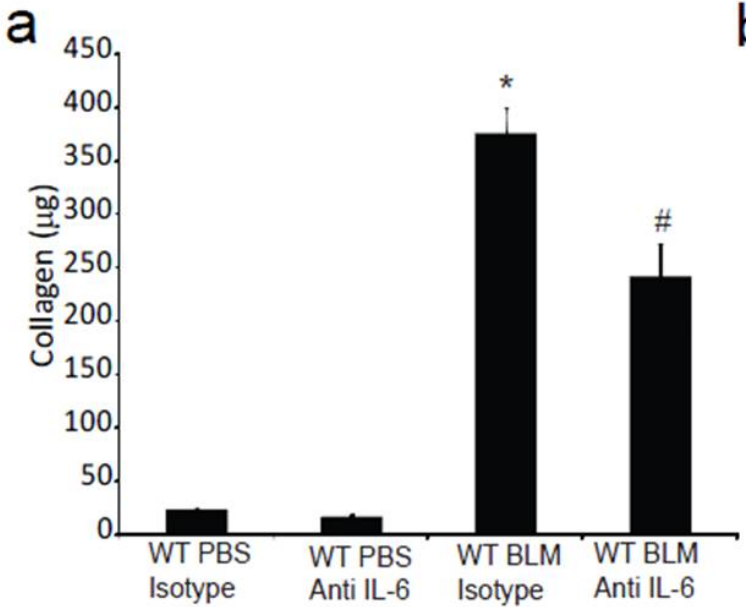

b
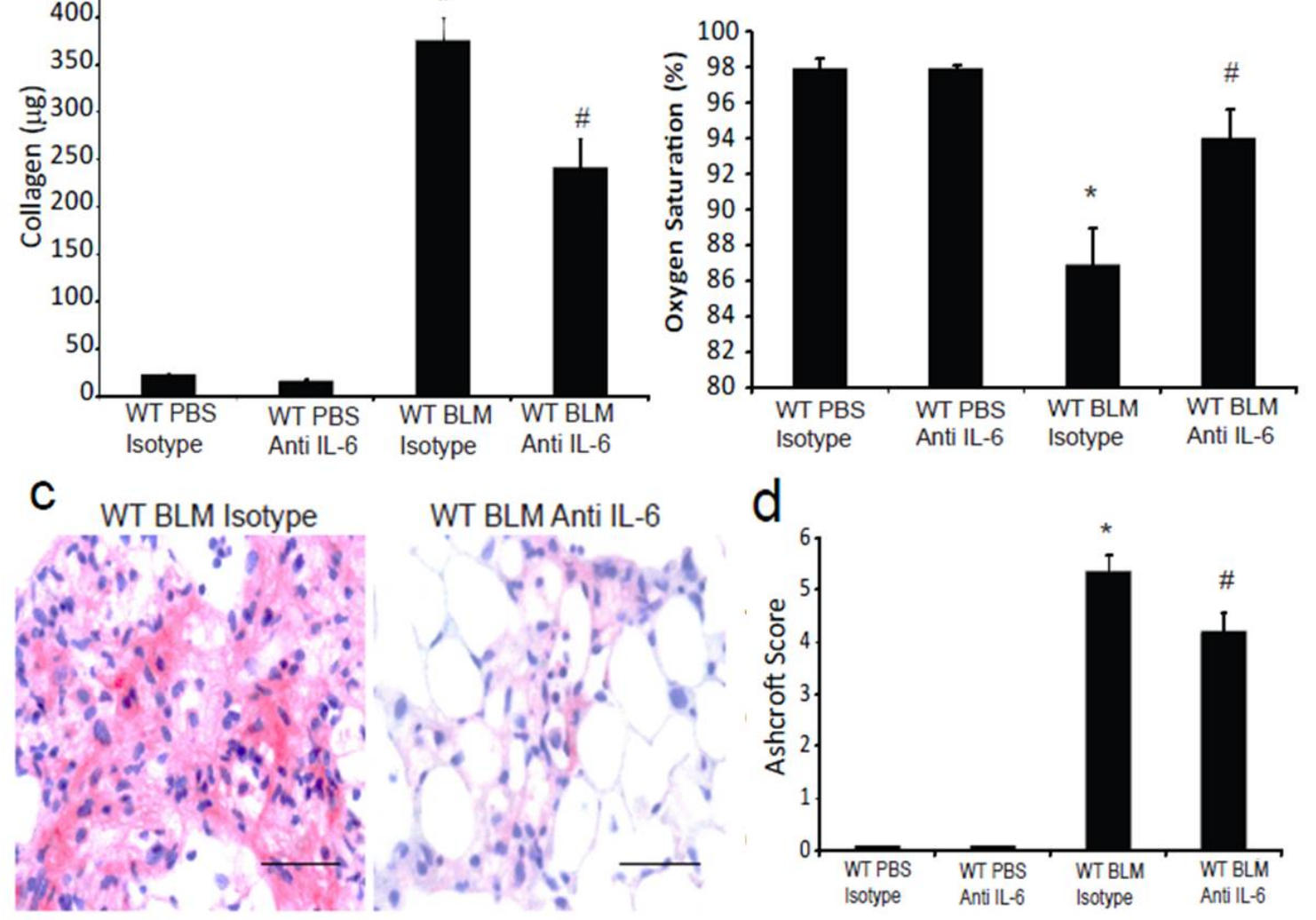

Figure 4.8

Pulmonary fibrosis following intraperitoneal bleomycin exposure in mice treated with IL-6 neutralization antibodies.

(a) Soluble collagen protein levels were measured using Sircol Assay. Data presented as mean $\mu \mathrm{g}$ collagen $/ \mathrm{ml}$ BAL fluid \pm SEM, $n \geq 8$. $\left({ }^{*}, p \leq 0.05\right.$ PBS $+\mathrm{IL}-6$ Antibody vs. Bleomycin + Isotype and \#, $p \leq 0.05$ Bleomycin + Isotype vs. Bleomycin + IL-6 Antibody). (b) Measurement of arterial oxygen saturation. Data presented as percentage of oxygen saturation \pm SEM, $n \geq 10\left(^{*}, p \leq 0.05\right.$ PBS + IL-6 Antibody vs. Bleomycin + Isotype and \#, p $\leq 0.05$ Bleomycin + Isotype vs. Bleomycin + IL-6 Antibody). (c) Lung sections were stained with an antibody against for $\square$-SMA to visualize myofibroblast accumulation (pink stain). Images are representative of 6 animals from each group. Scale bars: $100 \mu \mathrm{m}$. (d) Ashcroft scores were used to determine the degree of fibrosis $\left({ }^{*}, p \leq 0.05\right.$ PBS + IL-6 Antibody vs. Bleomycin + Isotype and \#, p $\leq 0.05$ Bleomycin + Isotype vs. Bleomycin + IL-6 Antibody, $\mathrm{n} \geq 6$ ). 

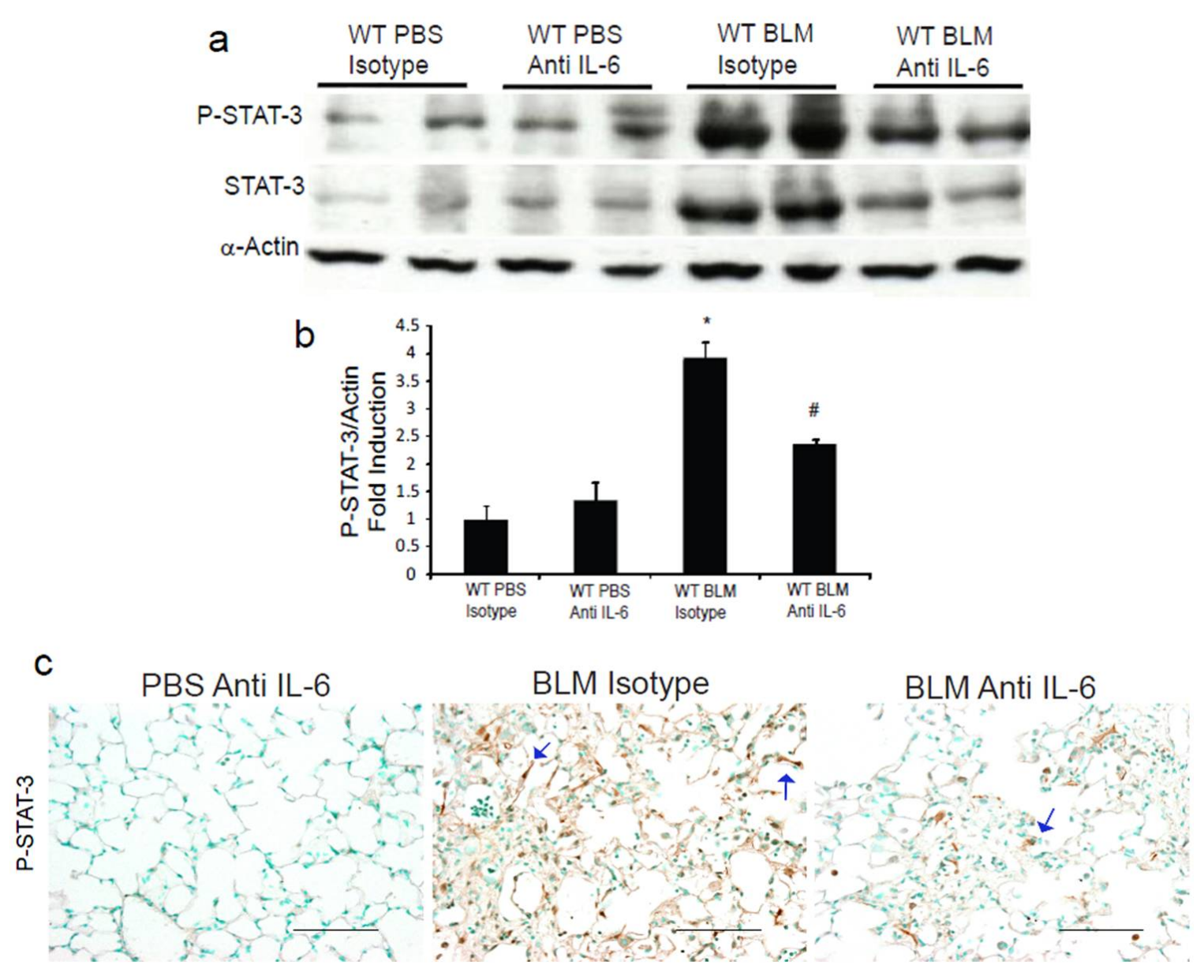

Figure 4.9

STAT-3 activation following neutralization of IL- 6 in bleomycin treated mice.

Phospho-STAT-3 expression. (a) Western Blot analysis was performed using an antibody against phospho-STAT-3 in whole lungs lysates. STAT-3 and $\alpha$-actin were used as controls. (b) Phospho-STAT-3 band intensity was quantified using ImageJ analysis. Values are presented as the percentage of $\alpha$-actin \pm SEM, $\mathrm{n} \geq 4$. ( ${ }^{*}, \mathrm{p} \leq \mathrm{PBS}+\mathrm{IL}-6$ Antibody vs. Bleomycin + Isotype and \#, $\mathrm{p} \leq 0.05$ Bleomycin + Isotype vs. Bleomycin + IL-6 Antibody). (c) Immunolocalization of phospho-STAT-3 expression. Lung sections were stained with an antibody specific for phospho-STAT-3 on alveolar epithelial cells (blue arrows). Images are representative of 8 animals from each group. Scale bars: $100 \mu \mathrm{m}$. 
cells. Moreover, this activation was reduced following IL-6 neutralization (Fig 4.9c). These results indicate that IL-6 acts as a pro-fibrotic cytokine in this model and contributes to the development of pulmonary inflammation and fibrosis, and neutralization of IL-6 is associated with the reduction of fibrotic features and STAT-3 activation in alveolar epithelial cells.

\section{DISCUSSION}

In this chapter, the contribution of IL-6 to pulmonary fibrosis was demonstrated in the i.p bleomycin model. Compared to other methods of bleomycin administration, the i.p. bleomycin model develops pulmonary fibrosis that resembles more adequately what is observed in human disease. Repeated i.p. bleomycin injections develop mild acute injury and progressive and irreversible pulmonary fibrosis. In Chapter 3 , the focus was on the determination of the contribution of IL-6 to pulmonary phenotypes in $A d a^{-/}$mice. It was demonstrated that IL-6 was able to regulate pulmonary inflammation, airspace destruction, and pulmonary fibrosis. However, to further corroborate the role of IL-6 in pulmonary disorders, the implementation of a second disease relevant model was necessary. For this reason, the contribution of IL-6 in the i.p. bleomycin model was determined. Genetic studies determined that $I L-6^{-/-}$mice exposed to bleomycin exhibit attenuation of pulmonary fibrosis. Moreover, to test the pharmacological approach, bleomycin-exposed mice were treated with IL-6 neutralizing antibodies. This therapeutic approach indicated significant efficacy of neutralizing IL-6 in the bleomycin model during established fibrosis. This 
demonstrated that neutralization of IL-6 is able to attenuate the development and progression of pulmonary fibrosis in the bleomycin model.

Patients with IPF exhibit varying degrees in inflammation and airway fibrosis with excessive fibroblast proliferation, myofibroblast differentiation, and extracellular matrix deposition (157). IPF is characterized by the development of fibroblastic foci and repetitive alveolar epithelial injury, which culminates in regenerative hyperplastic type II and loss of type I pneumocytes. Myofibroblast accumulation is thought to arise from proliferation and differentiation of resident fibroblasts, from bone-marrow derived circulating fibrocytes that serve as progenitors for interstitial fibroblasts, and from the process known as epithelial-tomesenchymal transition (EMT). EMT refers to the process where epithelial cells are capable of undergoing a reversible switch from an epithelial to mesenchymal, fibroblast-like phenotype resulting in $\alpha$-SMA expression, ECM deposition, myofibroblast accumulation, and fibrosis (158). We demonstrated that IL-6 deficiency and neutralization reduced pulmonary fibrosis in both $\mathrm{Ada}^{-/-}$mice and the bleomycin model. Our findings support previous statements implicating IL-6 in the regulation of fibrosis. In response to aerosolized antigen, IL-6 deficiency attenuates subepithelial fibrosis independently of inflammation (159). Also, transgenic mice overexpressing IL-6 in the airways develop subepithelial fibrosis, myofibroblast hyperplasia, and airway thickening (120). IL-6 is also able to induce hepatic myofibroblast proliferation (160). In the i.t. bleomycin model, IL-6 deficiency attenuates fibrotic distortion of lung architecture, decreases collagen content, and downregulates TGF- $\beta$ and MCP-1 (124). Based on our findings, IL- 
6 deficiency and neutralization in both models is associated with decreased macrophages and neutrophils, which could in part explain the attenuation in fibrotic changes since these inflammatory cells are known to produce pro-fibrotic mediators. In addition, different CC chemokines, such as MCP-1 and MCP-5, have been implicated in the trafficking of fibrocytes and contribution of fibrosis (161). Downregulation of MCP-1 and other chemoattractants via IL-6 neutralization provide a possible explanation of the attenuation of fibrosis.

Another possible molecular pathway by which IL-6 effects fibrosis include its role on fibroblasts. IL-6 promotes growth-arrest in fibroblasts from normal patients, whereas in fibroblasts from IPF patients IL-6 induces proliferative and anti-apoptotic effects (126), suggesting that IL-6 may inhibit fibrosis in normal conditions but in the setting of fibrosis IL-6 exacerbates disease conditions. Furthermore, it has been shown that activation of $A_{2 B} R$ in human lung fibroblasts leads to IL-6 production inducing myofibroblasts differentiation (28). This adenosine-dependent myofibroblast differentiation process was also prevented using an IL-6 neutralizing antibody. Another study demonstrated that exposure of cardiac fibroblasts with IL-6, and the sIL-6R $\alpha$ leading to IL-6 trans-signaling, promotes collagen production and myofibroblast differentiation (162). Furthermore, studies in IL-6 deficient mice upon wound exposure demonstrated that IL-6 modulates $\alpha$-SMA expression on dermal fibroblasts (163); and that IL-6 has the ability to modulate TGF- $\beta$ and TGF- $\beta$ R2 in the skin and dermal fibroblasts suggesting an association in fibrotic pathologies between IL-6 and TGF- $\beta$ (164). Thus, these previous reports and our current findings further 
corroborate the role of IL- 6 on fibrosis. The involvement of IL-6 in myofibroblast differentiation and extracellular matrix deposition is a possible mechanism by which IL-6 regulates the pathogenesis or maintenance of pulmonary fibrosis. Thus, it is conceivable that IL-6 contributes to the progression of pulmonary fibrosis as a pro-inflammatory cytokine by modulating immune responses that augment fibrosis and as a pro-fibrotic cytokine by exerting direct effects on target cells such as myofibroblast differentiation and extracellular matrix production. Our findings implicate a direct role by which distorted IL-6 signaling, be it classical or trans-signaling, or a combination of both, contribute to profibrotic effects that exacerbate pulmonary fibrosis.

In conclusion, the results presented in this chapter suggest that IL-6 regulates aspects of bleomycin-induced and adenosine-mediated pulmonary fibrosis. Furthermore, the therapeutic approach presented in this chapter indicates that IL-6-based therapies might be beneficial for patients with pulmonary fibrosis. In addition, STAT-3 activation points to a potential mechanism in alveolar epithelial cells by which IL-6 mediates pulmonary fibrosis. We need to conduct further studies in vitro to investigate the mechanisms by which IL-6 is able to induce fibrosis. Additionally, the findings in our models regarding the role of IL-6 in regulating fibrosis need to be translated into human disease for clinical relevance. These important aspects will be addressed in the next chapter. 


\section{CHAPTER FIVE}

INVESTIGATION OF MECHANISMS

OF IL-6 MEDIATED PULMONARY

FIBROSIS 


\section{INTRODUCTION}

\section{PULMONARY FIBROSIS AND EPITHELIAL-TO-MESENCHYMAL TRANSITION (EMT)}

Our understanding of the mechanisms underlying the pathogenesis of pulmonary fibrosis remains elusive. Originally it was believed that the development of pulmonary fibrosis was the result of chronic inflammation. This was accepted because the existence of immune-derived secreted factors that stimulate fibroblast growth, such as transforming growth factor (TGF)- $\beta$ and platelet-derived growth factor (PDGF) (165). However, it is now evident that inflammation and fibrosis are often not directly associated. Therefore, subsequent theories speculate that without preceding inflammation, fibrosis develops as a result of constant epithelial injury and from an impaired wound repair process (157). Following tissue injury, inflammatory and host tissue repair responses are evoked to initiate recruitment, activation, apoptosis, and clearance of effector cells. Yet, fibrosis is now thought of as the consequence of a dysregulated repair processes that are constitutively "turned on" while the inflammatory response is either reduced or "switched off" (2). Hence, these repair processes remain dysregulated while inflammation ceases at chronic fibrotic stages.

Pulmonary fibrosis can develop progressively as the result of constant lung injury or it can arise spontaneously from an unknown cause, hence the name idiopathic pulmonary fibrosis (IPF). Sustained alveolar epithelial damage leads to respiratory failure due to severe distortion of lung architecture. This lung 
architectural damage drives enhanced myofibroblast proliferation and activation resulting in excessive extracellular matrix (ECM) deposition in the lung interstitium (158). The myofibroblast is considered to be the primary effector cell of pulmonary fibrosis. However, the origin of the myofibroblast in the injured lung is not clearly established. Three potential sources have been hypothesized for the observed myofibroblast accumulation and differentiation. These are: i) proliferation and conversion of resident lung fibroblasts into myofibroblasts; ii) bone-marrow derived circulating fibrocytes, which serve as progenitors for interstitial fibroblast and differentiate into myofibroblasts; and iii) a process known as "epithelial-mesenchymal transition" (EMT) resulting in myofibroblast accumulation from epithelial progenitors. These different sources of myofibroblasts support the notion that fibrosis occurs independently of inflammation as the result of an abnormal wound healing response and constant epithelial injury.

The third hypothesis states that the contribution from the process known as EMT results in myofibroblast accumulation in the lung. In the context of pulmonary fibrosis, EMT is a process where type II alveolar epithelial cells (AECs) are capable of undergoing a form of metaplasia by switching from an epithelial to fully differentiated mesenchymal, fibroblast-like phenotype (166) (Fig 5.1). For clarity, type II AECs are able to differentiate into type I AECs to allow for re-epithelialization, a process known as trans-differentiation (167). It is important to note that the EMT process is involved in cellular transdifferentiation during development and tumor progression. During development, epiblasts 
undergo EMT to form primary mesenchyme and secondary epithelia are created through EMT in order to form fully differentiated adult epithelia or other cell types (168). During tumor progression, through EMT, epithelial cells are able to lose polarity, disassemble cell adhesion systems, produce cell-motility systems, and change location (169).

In the setting of pulmonary fibrosis, EMT is involved in the fibrotic processes by promoting the loss of the epithelial phenotype and favoring myofibroblast differentiation. TGF- $\beta$ is known to induce $\alpha$-smooth muscle actin ( $\alpha$-SMA) expression, serving as a key indicator of myofibroblast differentiation from fibroblasts. TGF- $\beta$ is an important cytokine that regulates cell growth, morphogenesis, cell differentiation, apoptosis, (170) and fibrosis (171). TGF- $\beta$ is recognized as a "master switch" of fibrosis. In addition to TGF- $\beta$, soluble growth factors such as epidermal growth factor (EGF), hepatocyte growth factor (HGF), and fibroblast growth factors (FGF), have also been demonstrated to play a role in EMT (172). Nevertheless, upon the influence of these factors EMT occurs in a reversible manner. However, only prolonged exposure of TGF- $\beta$ is able to induce complete EMT (172). In epithelial cells, treatment with TGF- $\beta$ leads to activation of transcription factors, such as Smads, Slug, Snail, Scatter, lymphoid enhancing factor-1 (LEF-1), and $\beta$-catenin (168). TGF- $\beta$ can also activate nonSmad mediated cellular signaling pathways, such as Rho kinase to rearrange the cytoskeleton and down-regulate E-cadherin (173). Thus, the "EMT proteome" is activated to induce junctional disassembly, cytoskeletal rearrangement, and cellular motility (174). 


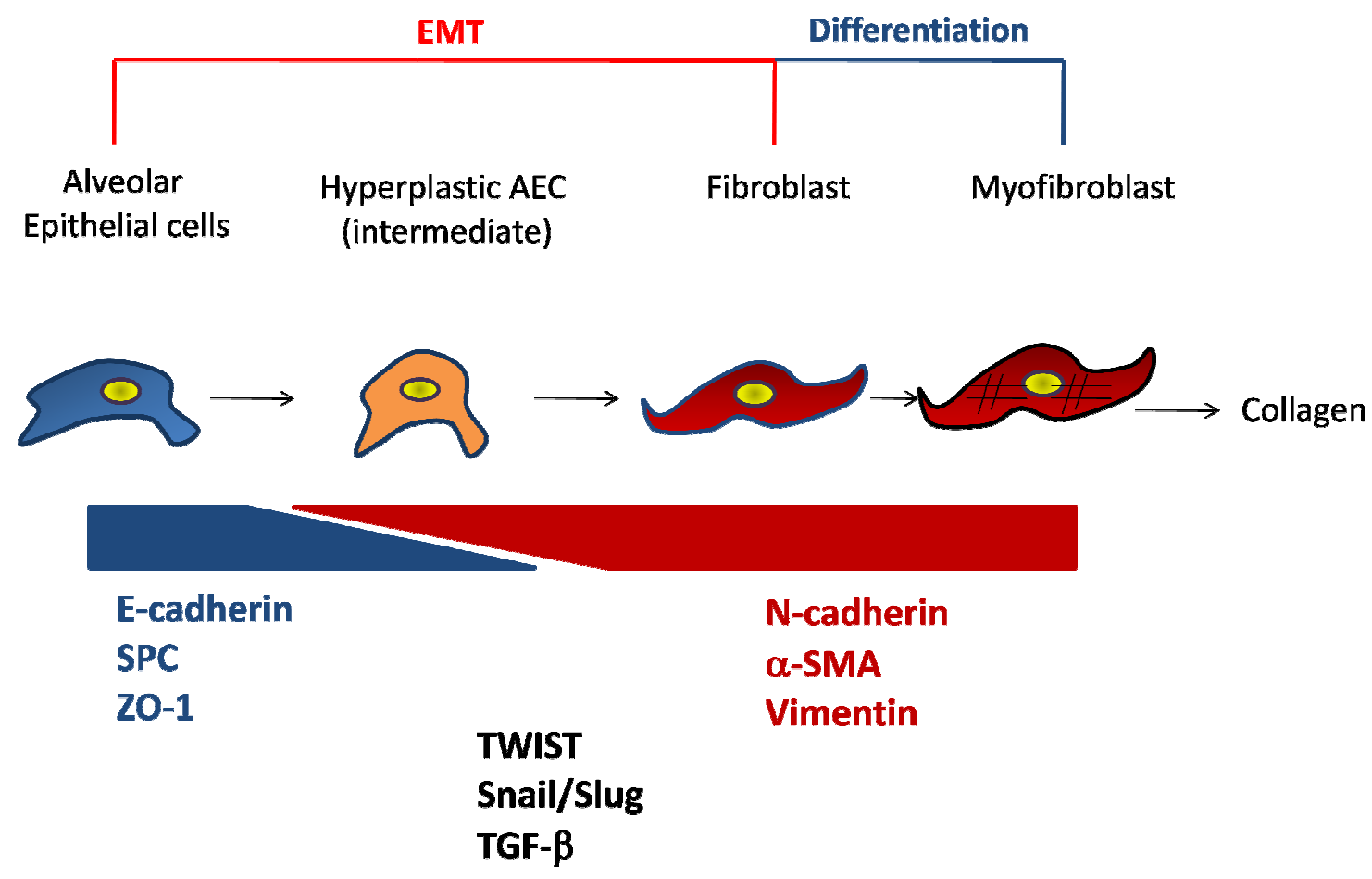

Figure 5.1

\section{Epithelial to Mesenchymal Transition (EMT)}

Diagram illustrating the cellular program EMT characterized by loss of cell adhesion, repression of E-cadherin expression, and increased cell mobility. EMT is essential for biological and disease processes including mesoderm formation, neural tube formation, tumor metastasis, and fibrosis. This diagram shows the graded response during which epithelial cells are transformed into mesenchymal cells. Mediators of EMT include TGF- $\beta$, Wnt $/ \beta$-catenin, and the Notch pathway. Master regulators of EMT include transcription factors Twist, Snail, Slug, and STAT-3. EMT markers include downregulation of E-cadherin (E-cad), surfactant protein-C (SPC), and zonula occludens-1 (ZO-1) and upregulation of N-cadherin, $\alpha$-smooth muscle actin ( $\alpha$-SMA), vimentin, fibronectin, and fibroblast-specific protein-1 (FSP-1). Differentiation of mesenchymal cells into myofibroblasts is characterized by $\alpha$-SMA expression. Myofibroblasts are the main cell type responsible for the production of the extra cellular matrix (ECM), mainly collagen. 
In addition, cross-talk between classical pathways (TGF- $\beta$ and Rho) and other signaling molecules (Ras, ERK, MAPK, Notch, Wnt proteins, NF-к $\beta$, and PI3K) have been implicated to play a role regarding the effect or reversibility of EMT in the fibrotic response (174). Despite the extensive studies regarding EMT and TGF- $\beta$ in fibrosis, there is still a knowledge gap in the pathobiology and biogenesis of pulmonary fibrosis that renders this disorder untreatable. Thus, to address this knowledge gap in pulmonary fibrosis, in this dissertation I focus on an alternative signaling pathway that could promote the accumulation of the fibroblasts and myofibroblasts in the injured lung. This chapter focuses on the role of IL-6 trans-signaling in mediating EMT and fibrosis.

The identification of fibroblasts and myofibroblasts that have differentiated from epithelium consists of analysis in morphologic changes, acquisition of mesenchymal phenotype, and loss of epithelial characteristics. The morphological change in EMT consists of the modification from a cuboidal cell shape (AEC II) to an elongated or spindle-shaped form (fibroblast/myofibroblast). The acquisition of fibroblast- or myofibroblast-specific markers consists of fibroblast specific protein (FSP-1) and $\alpha$-SMA, respectively. The loss of epithelial phenotype includes down-regulation of E-cadherin, cytokeratins, surfactant protein C (SPC), thyroid transcription factor-1 (TTF-1), and zonula occludens-1 (ZO-1) (175). Regarding differentiation, the myofibroblast is the intermediate cell type between the fibroblast and smooth muscle cell. To distinguish fibroblast from myofibroblasts it is important to note that fibroblasts might contain actin in their cortex similar to proto-myofibroblasts; however, fibroblasts neither express 
stress fibers nor they are able to form adhesion with the extracellular matrix (176). Thus, these are known as non-contractile fibroblasts. Another characteristic feature is that fibroblasts express vimentin, but not desmin and $\alpha$ SMA (177). Fibroblasts differentiate upon mechanical stress into protomyofibroblasts, which contain cytoplasmic actin-containing stress fibers terminating in fibronexus adhesion complexes, and are able to express cellular fibronectin and generate contractile force (176). Stimulation of TGF- $\beta$ increases ED-A fibronectin expression, which under mechanical stress will promote the differentiation of proto-myofibroblasts into myofibroblasts. These myofibroblasts are characterized by de novo $\alpha$-SMA expression, extensively developed stress fibers, increased fibronexus adhesion complexes, greater contractile force, and higher organization of extracellular fibronectin into fibrils (176). Due to the heterogeneity in these cells, other markers to distinguish the myofibroblast include vimentin, yet $\alpha$-SMA is the most widely accepted. Although $\alpha$-SMA expression is used to distinguish the myofibroblast, $\alpha$-SMA is the most abundant protein in smooth muscle cells. Distinction between the smooth muscle cell and the myofibroblast can be made due to the following: myofibroblasts have a surface characterized by fibronectin fibrils and fibronexus junctions, whereas the external lamina (basement membrane), attachment plaques, and plasmalemmal caveolae are typical of smooth muscle cells (177). In addition, myofibroblasts can also be distinguished due to their lack of smooth muscle markers, such as desmin and smooth muscle myosin (178). Thus, myofibroblasts identification consists of abundant rough endoplasmic reticulum, peripheral myofilaments with 
focal densities (stress fibers), fibronexus junctions, and expression of $\alpha$-SMA and vimentin (177).

The fibroblasts are spindle shaped cells found in many tissues and organs. Fibroblasts respond to and synthesize various cytokines, chemokines, and other mediators of inflammation, such as TGF- $\beta$-and PDGF (179). Fibroblasts also participate in the wound healing responses by producing ECM molecules, such as collagens, proteoglycans, fibronectin, tenascin, and laminin (178). Fibroblasts also produce matrix metalloproteinases (MMPs) and tissue inhibitors of metalloproteinase (TIMP). The production of these proteases and anti-proteases participate in the regulation of the ECM degradation (178). In addition, fibroblasts also regulate tissue interstitial fluid volume and pressure via interaction of $\beta 1$ integrin receptors. Upon activation following tissue injury, fibroblasts differentiate into myofibroblasts. Myofibroblasts in turn play a central role in wound healing and repair by participating in the physiological reconstruction of connective tissue after injury (180). Myofibroblasts express $\alpha$ SMA containing stress fibers, whereby exert contractile force to reduce the size of the wound. The final phases of remodeling consist of resolution of MMPs and TIMPs, ECM degradation, and myofibroblasts removal by apoptosis (178). Dysregulation of this repair mechanism leads to an abnormal and pathological wound healing response. Thus, the dysregulation of injury repair response and myofibroblast function are responsible for generating the pathological tissue deformations, which are characteristic of fibrosis. This dysregulation is responsible for myofibroblast accumulation. In turn, the myofibroblast is 
responsible of increased ECM deposition, altered tissue architecture, and impaired function (179).

Many fibrotic diseases are associated with abnormal repair responses due to imposed tissue injury, such as traumatic/mechanical, environmental, infectious, cancerous, autoimmune, and/or drug-induced insults. It is believed that myofibroblasts play a central role in the pathogenesis of idiopathic pulmonary fibrosis (IPF). The pathogenesis of IPF is associated with increased numbers of fibroblastic foci and rapid development of fibrotic lesions, which are composed of proliferating myofibroblast. These myofibroblasts are key mediators of ECM deposition, structural remodeling, and destruction of alveocapillary units upon lung injury (181). The myofibroblast contributes to active fibrosis by its ability to express increased levels of collagen, contributing to high levels of ECM that constitutes tissue destruction and scar acquisition (181). Besides the role ECM deposition, myofibroblasts are a key source of fibrotic cytokines (TGF- $\beta$ ) and pro-inflammatory cytokines, such as the CC chemokine, monocyte chemotactic protein-1 (MCP-1). Thus, this amplification of inflammation may result in a positive feedback loop intensifying the progression of fibrosis (181). Another important aspect of the myofibroblast is its contractility, which is important in wound contraction and is responsible for the altered mechanical characteristic in pulmonary fibrosis (182). Therefore, the persistence of myofibroblasts within a fibrotic lesion leads to the functional impairment of the affected organ due to the excessive scarring, which creates a honeycombing appearance (183). Thus, dysregulation of fibroblast/myofibroblast function is 
associated with features that affect tissue structure and function such as excessive ECM deposition and inappropriate tissue contraction. Hence, understanding this dysregulation is critical to develop novel and effective therapies that address not only the symptoms but the progressive nature of pulmonary fibrosis. In this dissertation, the role of IL-6 was determined as an alternative pathway involved in the development of pulmonary fibrosis.

\section{IL-6 IN PULMONARY FIBROSIS}

Significant efforts are conducted in order to identify significant roles of growth factors and cytokines involved in disease pathogenesis. Thus, the role of the IL-6/gp130 family of cytokines has been suggested to play a role in the susceptibility and progression of pulmonary fibrosis (122). Cytokines such as leukemia inhibitory factor (LIF), oncostatin M (OSM), IL-11, ciliary neurotrophic factor (CNTF), and cardiotrophin-1 (CT-1) belong to the IL-6 family of cytokines. These cytokines are comprised of a single chain polypeptide ( 20 kb), which all engage the signal transducing unit gp130 (90). The family of suppressor of cytokine signaling (SOCS) proteins is the main inhibitor of gp130 signaling via a classical negative feedback loop (184). IL-6 is a pleiotropic cytokine with relevant effects on inflammation and fibrosis, yet its role in fibrosis remains unknown. Profibrotic growth factors (i.e. TGF- $\beta$ ) are able to induce IL-6 release, which mediated cellular effects that might be attributed to the inductors of IL-6 (185). Regarding the fibrotic role of IL-6, studies have demonstrated that IL-6 is able to induce collagen production from dermal fibroblasts (125). Also, patients 
with systemic sclerosis demonstrate increased IL-6 production by mononuclear cells (186). Human lung fibroblasts isolated from IPF patients respond abnormally to IL-6, which display mitogenic properties via sustained activation of ERK resulting in p27Kip1 inhibition and induction of cyclin D1 (187). The response of normal fibroblasts to IL-6 was the opposite, mainly sustained STAT3 activation and production of cyclin-dependent kinase inhibitor p19INK4D resulted in the inhibition of proliferation (187). Also, IL-6 is able to maintain normal fibroblasts in cell cycle arrest due to increase Bax expression, which promotes apoptosis (145). However, in IPF fibroblasts, IL-6 is able to suppress apoptosis by inducing the expression of $\mathrm{Bcl}-2$, which is an anti-apoptotic protein (187). Thus, IL-6 is able to have various effects on normal cells and IPF fibroblasts depending on the pathway activated, either ERK or STAT-3, and the kinetics of the activation, either transient or sustained. For example, in normal cells induces apoptosis via transient ERK and sustained STAT-3 activation; whereas in IPF fibroblasts, proliferation was induced via sustained ERK and transient STAT-3 activation (187). These observations indicate that IL-6 signaling via STAT-3 activation is dysregulated in human lung disease. Thus, investigations of IL-6 signaling pathways are critical to further understand the pathogenesis of chronic lung diseases.

Furthermore, mice overexpressing IL-6 in the lung are characterized with a diffuse peribronchial mononuclear cell inflammatory response (188). Although these mice are resistant to airway hyperresponsiveness, minimal signs of fibrosis are observed. Similar findings were observed in rats overexpressing IL-6 in the 
airways, which induced lymphocytic alveolitis without proliferation of fibroblasts (189). However, since IL-6 is an important cofactor in inflammatory cell recruitment and activation, indirect fibrotic contributions are easily overlooked. For example, IL-6 and TNF $\alpha$ together are able to regulate the expression of MIP1 $\alpha$, which is a profibrotic chemokine (123). In the bleomycin model of pulmonary fibrosis, IL-6 is secreted by endothelial cells and macrophages in rats $(190,191)$. However, the role of IL-6 and its interaction with other mediators at the setting or during the development of lung injury remains unknown. As noted in previous chapters, IL-6 neutralization was shown to reduce fibrosis. However, this effect was not demonstrated to be a direct cause of IL-6 neutralization. This was the case since reduction of fibrosis via IL-6 neutralization was observed in conjunction with diminished levels of important chemokines and cytokines known to be mediators of fibrosis. Thus, analysis of direct mechanisms by which IL-6 induces fibrosis is addressed in this chapter.

\section{CLASSICAL VS IL-6 TRANS-SIGNALING}

In response to injury and infection, IL-6 is produced by different cells such as, mast cells, eosinophils, endothelial cells, smooth muscle cells, monocytes/macrophages, and B and T cells (88). In addition, IL-6 is able to induce autoimmunity, amplify acute inflammation, and develop chronic inflammation (89). Dysregulation of IL-6 signaling has been implicated in the onset and maintenance of several diseases, such as multiple sclerosis, inflammatory bowel disease, rheumatoid arthritis, and cancer (90). IL-6 signals 
by binding to the membrane-bound IL-6 receptor mIL-6R $\alpha$, which is expressed predominantly on leukocytes and hepatocytes, and then associates with the signal-transducing gp130 protein in order to phosphorylate and dimerize STAT-3. Thus, phospho-STAT-3 is an indicator of IL-6-induced cellular activation. Phosphorylated STAT-3, once dimerized, is translocated into the nucleus to act as a transcription factor and regulate the expression of target gene (101). STAT3 up-regulation and STAT-3 downstream targets also have been implicated in lung diseases $(98,102)$. This trans-signaling mechanism increases the spectrum of IL-6 activities from immune responses to involvement of chronic pathological states. Furthermore, elevations of the agonist sIL-6R $\alpha$ have been reported in cancer and several chronic inflammatory and autoimmune diseases (112).

Since the mIL-6R $\alpha$ is only expressed on leukocytes and hepatocytes, evaluation of the IL-6 trans-signaling is required for proper understanding of IL-6 biological activity. In the absence of mIL-6R $\alpha$ cells are unresponsive to IL-6. The sIL-6R $\alpha$ is able to associate with secreted IL-6 forming a complex with high affinity for gp 130 thereby acting as an agonist by increasing the spectrum of IL-6 activity (132). The signal transducing gp130 is ubiquitous expressed rendering cells otherwise unresponsive to IL-6 to become activated by the IL-6/sIL-6R $\alpha$ complex. Accordingly, the agonist sIL-6R $\alpha$ can be produced by two different mechanisms, by proteolytic cleavage (PC-sIL-6R $\alpha$ ) or by an alternatively spliced mRNA (AS-sIL-6R $\alpha$ ) (53). In addition, both IL-6 and the sIL-6R $\alpha$ are increased in numerous inflammatory diseases $(98,101,102)$. Thus, the $\mathrm{mIL}-6 \mathrm{R} \alpha$ is primarily associated with anti-inflammatory qualities, whereas the sIL-6R $\alpha$ has been 
associated in pathological conditions with pro-inflammatory properties (112). For instance, in breast cancer patients IL-6 elevations correlates with poor survival (192). Furthermore, it has been demonstrated that IL-6 through STAT-3 activation mediates EMT in breast cancer cells via trans-activation of TWIST, a known metastatic regulator (193). Although levels of IL-6 have been reported in chronic lung diseases, the role of IL-6 trans-signaling has not been fully investigated in particular in pulmonary fibrosis. The purpose of this chapter is to elucidate the mechanism by which IL-6 trans-signaling can induce fibrosis.

\section{SPECIFIC QUESTIONS AND EXPERIMENTAL RATIONALE}

In conjunction with increases in levels of adenosine and IL-6, $A d a^{-/-}$mice develop features of pulmonary fibrosis including the production and deposition of collagen (119). In addition, adenosine and its stable analog, 5'-(Nethylcarboxamido)-adenosine (NECA), are able to activate the $A_{2 B} R$ to increase

IL-6 release and induce the differentiation of human lung fibroblasts to myofibroblasts, which can be attenuated with treatment of an IL-6 neutralizing antibody (28). This suggests that IL-6 displays pro-fibrotic properties in the differentiation of fibroblast to myofibroblasts. Furthermore, the previous chapters have demonstrated a pro-fibrotic role of IL-6 by attenuation of fibrosis in Adamice and bleomycin-exposed mice subjected to genetic removal or neutralization of IL-6. Hence, the implied role of IL-6 in the differentiation of fibroblasts to myofibroblasts and the results from previous chapters indicate that IL-6 signaling is involved in regulating the fibrotic responses seen in $\mathrm{Ada}^{-/-}$mice and in the 
bleomycin model. However, the mechanism of action by which IL-6 confers fibrotic features remains unknown. In addition, increased STAT-3 activation on alveolar epithelial cells was attenuated in association with decreased collagen deposition and reduced $\alpha 1$-procollagen expression in both models subjected to genetic removal and neutralization of IL-6. To the best of our knowledge, alveolar epithelial cells do not express the mIL-6R $\alpha$ suggesting that IL- 6 transsignaling is inducing pro-fibrotic properties on these cells via STAT-3 activation. It has been shown that IL-6 trans-signaling induces EMT in cancer (193). This chapter will address the mechanism by which IL-6 confers fibrotic characteristics and the role of IL-6 trans-signaling on pulmonary fibrosis. Examination of EMT markers determined the role of $\mathrm{IL}-6$ in the pathogenesis at least in vitro. Furthermore, characterization of IL-6 trans-signaling in the lungs of patients with COPD and IPF corroborated the findings in the mouse models employed in the previous chapters. Hence, I demonstrate for the first time that IL-6 transsignaling represents a potential therapeutic application for pulmonary fibrosis.

\section{RESULTS}

\section{ELEVATIONS OF SIL-6R $\alpha$ IN MODELS AND PATIENTS WITH PULMONARY FIBROSIS}

IL-6 classical signaling involves the binding of IL- 6 to membrane bound IL$6 \mathrm{R} \alpha$ to initiate a conformational change that facilitates interaction with gp130 signaling receptors to promote downstream signaling via STAT-3 phosphorylation (90). This pathway is abundant in cells derived from the 
hematopoetic lineage and in hepatocytes (142). The IL-6 trans signaling pathway involves the generation of an alternative splice isoform or the shedding of the mIL-6R $\alpha$ to generated the sIL-6R $\alpha$. In turn, IL-6 binds the sIL-6R $\alpha$ and facilitates binding of this complex to gp130 on cells that lacks the mIL-6R $\alpha(98)$. This IL-6 trans-signaling pathway has been implicated in the amplification of various inflammatory and chronic diseases (141), but has not been examined in pulmonary fibrosis. To address this gap in our knowledge, an ELISA specific for sIL-6R $\alpha$ was used and revealed a marked increase in sIL-6R $\alpha$ levels in the lavage fluid from $\mathrm{Ada}^{-/}$mice and mice exposed to bleomycin (Fig. 5.1). Based on these observations, we hypothesized that IL-6 trans-signaling promotes EMT in airway epithelial cells through a pathway that involves STAT-3 activation.

\section{IL-6 TRAN-SIGNALING AND EMT IN HUMAN AND MURINE ALVEOLAR EPITHELIAL CELLS}

Elevations of sIL-6R $\alpha$ in $A d a^{-/-}$mice and in the bleomycin model indicate that IL-6 trans-signaling may be functional during disease conditions. Moreover, in both models increased activation of phospho-STAT-3 was observed mainly in alveolar epithelial cells (AECs) in the airways. Genetic removal and neutralization of IL-6 in both models was associated with decreased phosphoSTAT-3 in AECs and attenuation of fibrosis. Since mainly hepatocytes and leukocytes express the mIL-6R $\alpha$, these observations imply that the observed IL-6 dependent activation in AECs involves IL-6 trans-signaling. Thus, I hypothesized that IL-6 trans-signaling through STAT-3 activation promotes EMT in AECs. 

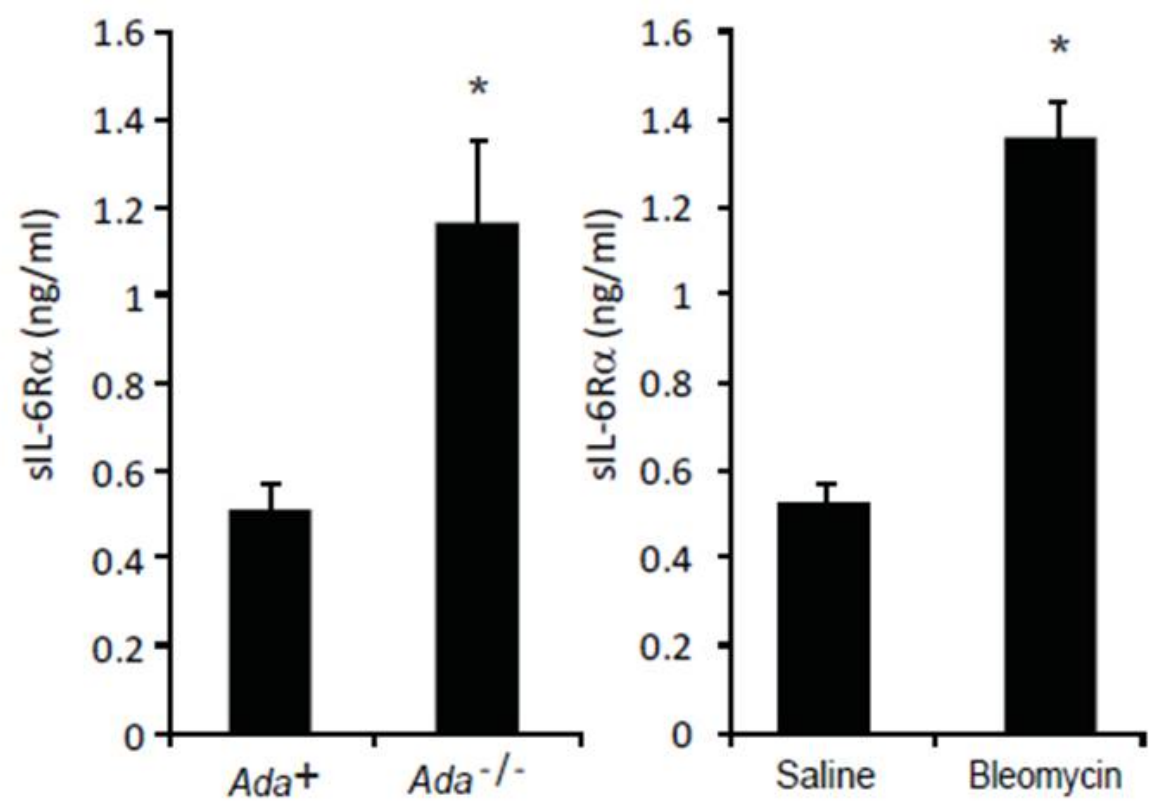

Figure 5.2

Elevation levels of sIL-6R $\alpha$ in ADA-deficient mice and bleomycin model.

(a) Measurements of sIL-6R $\alpha$ in $A d a^{-/}$mice and bleomycin exposed mice. Data

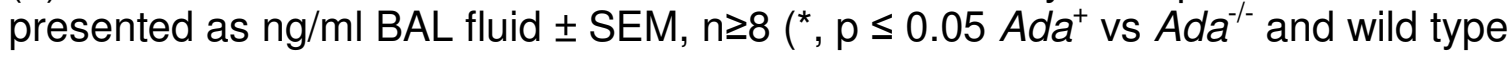
PBS vs wild type bleomycin). 
To address this, I directly exposed several airway epithelial cells lines (A549, MLE-12, and MLE-15) to IL-6 or slL-6R $\alpha$ together with IL-6 and examined STAT-3 activation and EMT markers. Results revealed that both IL-6 classical and trans-signaling could promote morphological changes in airway epithelial cells consistent with EMT (Fig. 5.3). Furthermore, western blot analysis of cell lysates revealed that both IL-6 and sIL-6R $\alpha$ exposure led to increased STAT-3 activation; however, sIL-6R $\alpha$ provided enhanced activation over that seen with IL-6 alone (Fig. 5.4). Examination of EMT markers revealed that both IL-6 and sIL-6R $\alpha$ could directly promote EMT with sIL-6R $\alpha$ exhibiting amplification of EMT relative to IL-6 alone. EMT was determined by decreased expression of E-cad and increased expression of mesenchymal markers such as vimentin and $\alpha$-SMA (Fig. 5.4). These findings suggest that IL-6 trans-signaling can directly promote EMT in AECs.

Examination of the signaling mechanism by which IL-6 regulates the induction of EMT in these cell lines revealed that TWIST activation was enhanced with exposure of both IL-6 and the sIL-6Ra (Fig 5.4). TWIST has been demonstrated to be a master regulator of EMT (194). Also, TWIST activation has been shown to play a prominent role in EMT in association with cancer metastasis (195). Furthermore, in breast cancer cell line, IL-6 was able to induce EMT via increased activation of TWIST (193). Hence, this indicates that IL-6 stimulation is associated with activation of TWIST and EMT induction. In this chapter, the results provided demonstrate that exposure of IL-6 and sIL-6R $\alpha$ to 

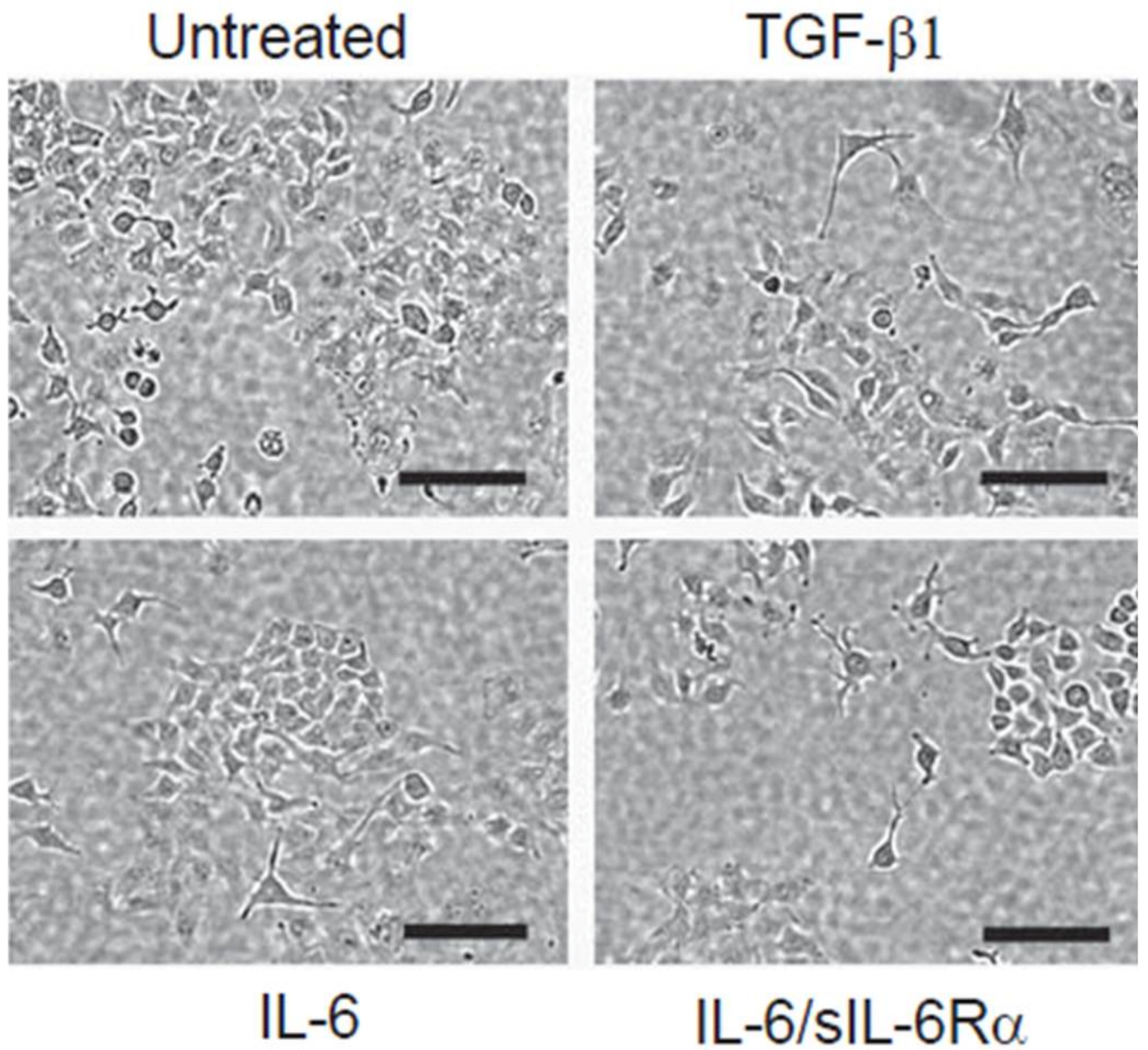

IL-6/sIL-6R $\alpha$

\section{Figure 5.3}

\section{Morphological changes induce by IL- 6 trans-signaling in cell culture studies}

MLE-12, MLE-15 and A549 airway epithelial cells were all used for the assessment of EMT. These cells were treated with media as negative control, TGF- $\beta$ as positive control. Experimental treatment included IL- 6 alone, sIL-6R $\alpha$ alone, and IL- 6 and sIL-6R $\alpha$ in combination to test the effect of IL-6 transsignaling. In this figure, MLE-12 cell lines are shown. Similar findings were observed for all cell lines (data not shown). 


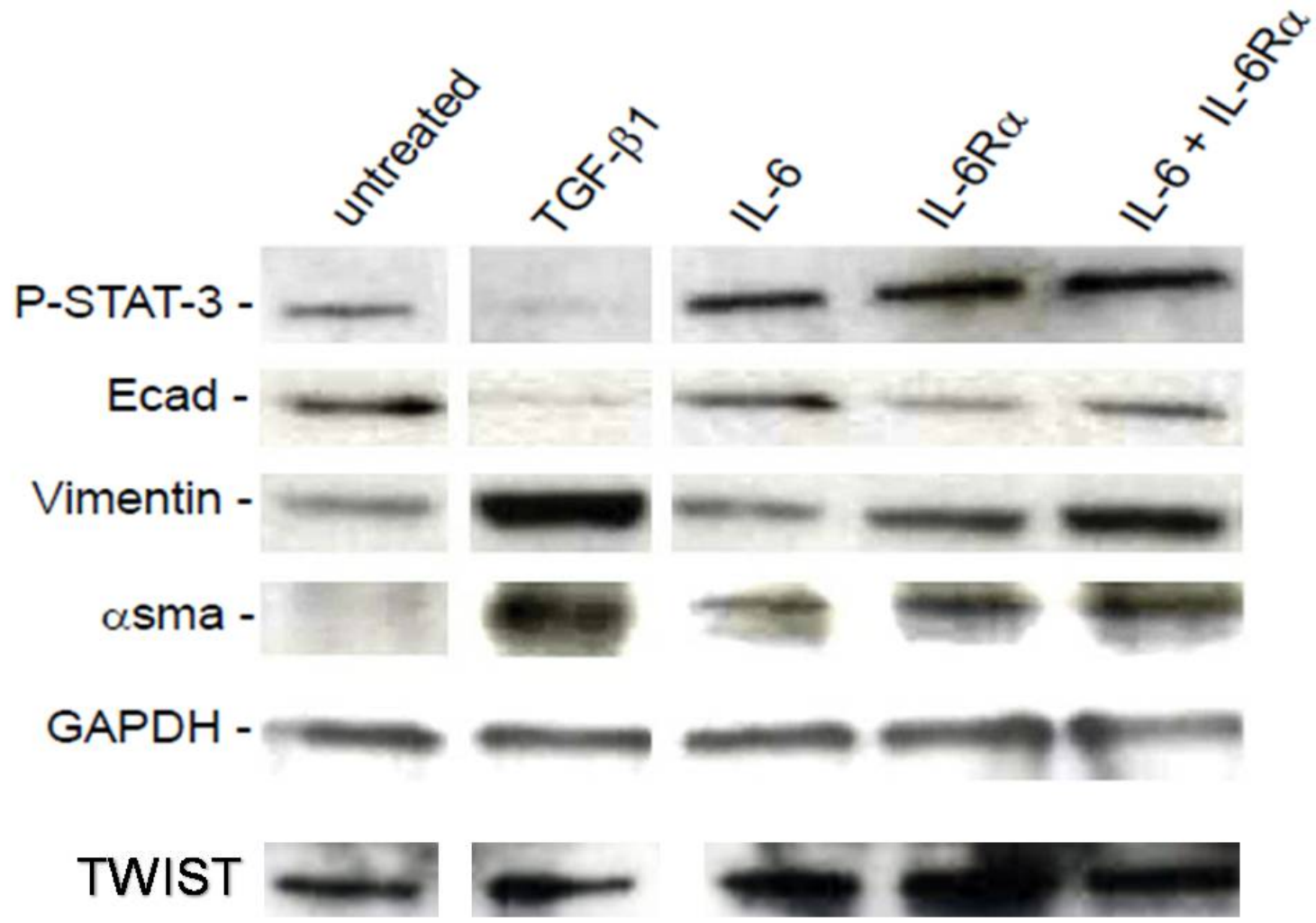

Figure 5.4

Characterization of IL-6 trans-signaling in cell culture studies.

MLE- 12 cells were treated with IL- 6 alone, sIL-6R $\alpha$ alone, and with IL- 6 and SIL$6 \mathrm{R} \alpha$ together. TGF- $\beta$ was used as the positive control for EMT. This figure is a representative of 10 different experiments where MLE-12 cell lines were exposed to the different agents for 72 hours. Western blot analysis for phospho-STAT-3, TWIST, and EMT markers on MLE-12 cells under no treatment or treatment with TGF- $\beta$, IL-6 alone, sIL-6R $\alpha$ alone or the IL-6/sIL-6R $\alpha$ complex. EMT markers included $\mathrm{E}$-cadherin (E-cad), vimentin and $\alpha$-smooth muscle actin ( $\alpha$-SMA). GAPDH was used as a loading control. 
alveolar epithelial cell lines led to EMT induction in conjunction with increased STAT-3 and TWIST activation (Fig 5.4). These results suggest that IL-6 transsignaling is involved in regulating fibrosis by enhancing EMT via activation of the transcription factors STAT-3 and TWIST.

\section{IL-6 EXPRESSION AND STAT-3 ACTIVATION IN PATIENTS WITH PULMONARY FIBROSIS}

The findings observed in both models, the $\mathrm{Ada}^{-/-}$mice and the bleomycin model, suggested that IL-6 is involved in the regulation of fibrosis. Also, in vitro studies indicated that IL-6 trans-signaling is able to enhance EMT induction. To determine if these findings are relevant to human disease, this signaling pathway was examined in tissue sections from patients of severe IPF in comparison with mild disease patients. Tissue sections from surgical biopsies obtained from the Lung Tissue Research Consortium were subjected to IL-6 and phospho-STAT-3 immunostaining. Patients with preserved lung function (FEV and FVC $>80 \%$ ) do not express IL-6 or phospho-STAT-3 in the alveolar airways (Fig 5.6a, left panels). However, in Stage 4 COPD patients (FEV < 50\%), IL-6 was expressed in inflammatory cells, mostly alveolar macrophages, whereas phospho-STAT-3 was detected in alveolar epithelial cells (Fig 5.6a, middle panel). In Severe IPF patients (FVC < 50\%), IL-6 was localized in hyperplastic epithelial cells in remodeled airways adjacent to fibroblast foci and phospho-STAT-3 nuclear localization was observed in hyperplastic alveolar epithelial cells (Fig6a, right panels). Western blot analysis on lung homogenates from the same patients 


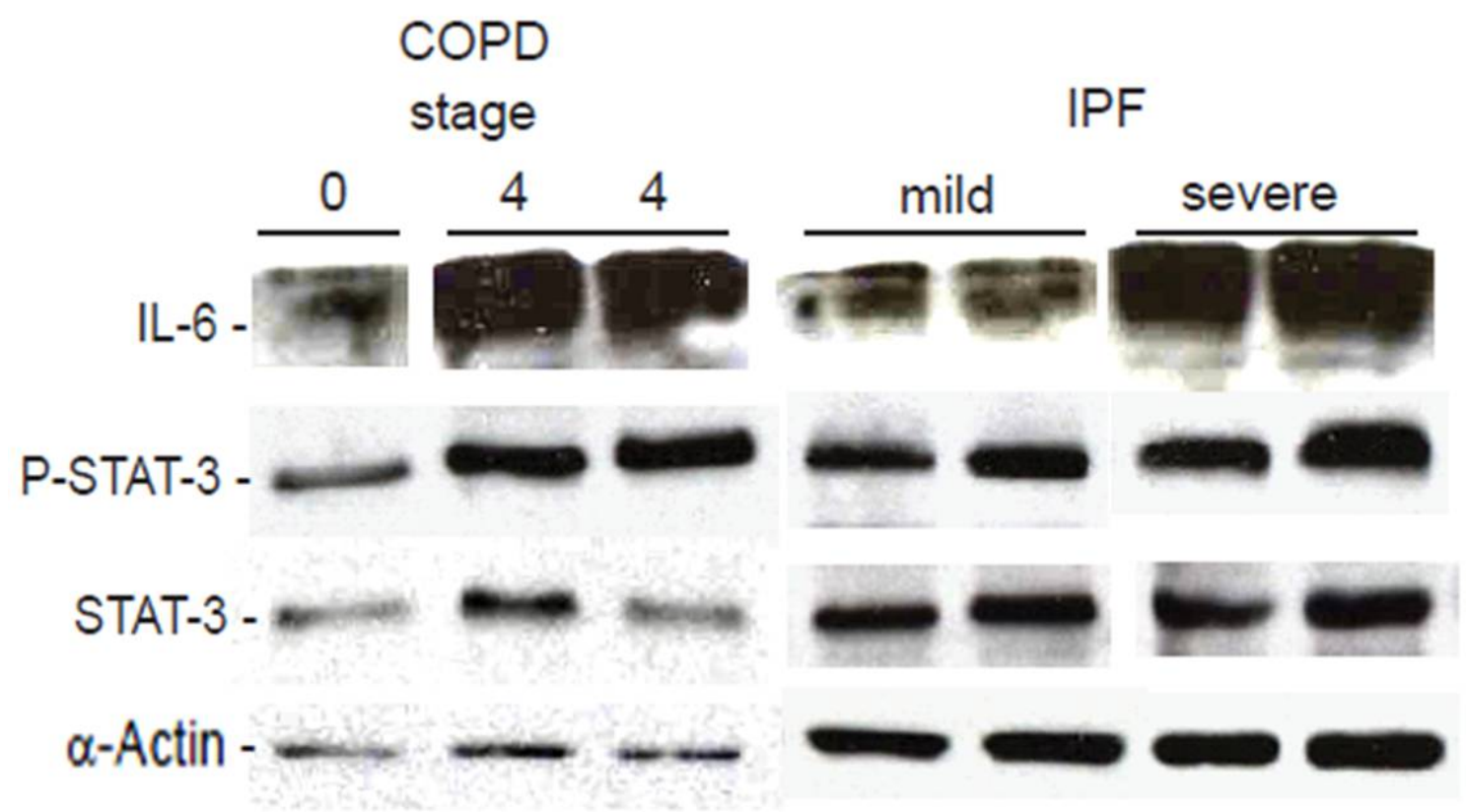

Figure 5.5

Characterization of IL-6 expression and STAT-3 activation in human IPF patients

Western Blot analysis for IL-6 and phospho-STAT-3 expression on human patients with stage 4 COPD and severe IPF. Tissue sections from surgical biopsies were obtained from the Lung Tissue Research Consortium. Patients with preserved lung function (FEV and FVC $>80 \%$ ) were used as normal. Stage 4 COPD patients (FEV < 50\%) and severe IPF patients (FVC $<50 \%$ ) were subjected to IL-6 and phospho-STAT-3 immunostaining. 


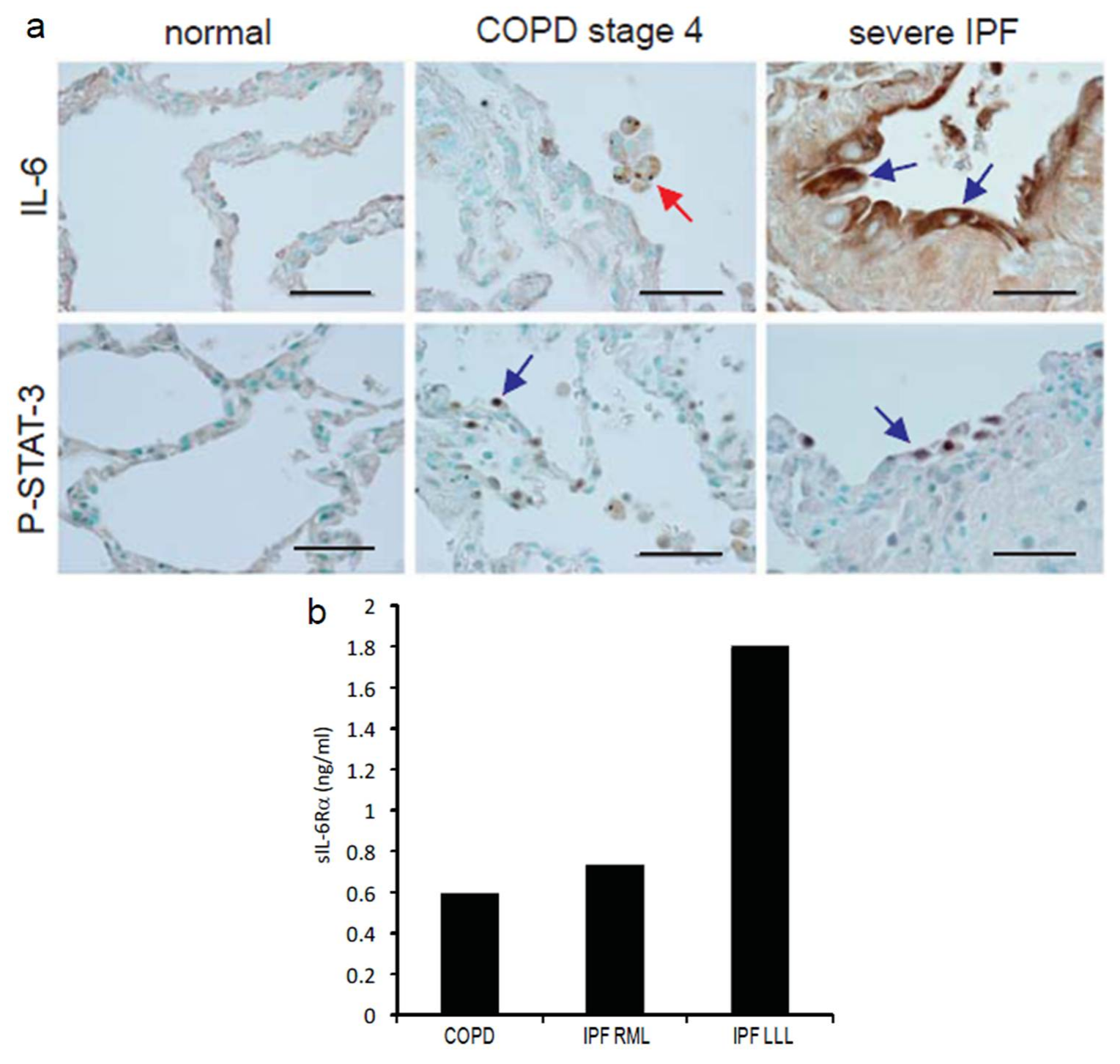

Figure 5.6

\section{Characterization of IL- 6 trans-signaling in human IPF patients}

(a) Immunolocalization of IL-6 and phospho-STAT-3 expression on alveolar macrophages (red arrows) and alveolar epithelial cells (blue arrows) in lung sections from patients of COPD stage 4 and severe IPF. Images are representative of 4 patients from each group. Scale bars: $50 \mu \mathrm{m}$. (b) Measurements of sIL-6R $\alpha$ in BAL fluid using ELISA in a COPD patient and from two different lobes from the same IPF patient, right middle lobe (RML) and lower left lobe (LLL). 
demonstrated increased expression of IL-6 and phospho-STAT-3 in both Stage 4 COPD and Severe IPF patients (Fig 5b). These results confirm the observations seen in mouse models and demonstrate that key components of IL6-signaling are elevated in patients with COPD and IPF.

In addition, we demonstrated that the sIL-6R $\alpha$ is elevated in patients of severe IPF. An ELISA specific for sIL-6R $\alpha$ was used and revealed a marked increase in sIL-6R $\alpha$ levels in the lavage fluid from a COPD patient and from two different lobes from the same IPF patient, right middle lobe (RML) and lower left lobe (LLL). Interestingly, in the IPF patient only the LLL demonstrated elevations of the sIL-6R $\alpha$. These results suggest that generation of sIL-6R $\alpha$ is locally produced at the site of injury and that IL-6 trans-signaling is associated with active disease.

\section{DISCUSSION}

Our findings demonstrate that increased elevations of IL-6 are associated with increased STAT-3 activation in AECs. Activation of STAT-3 in AECs has been previously reported (196-199). Conditional deletion of STAT-3 in type II AECs results in mice being susceptible to pulmonary damage once exposed to hyperoxia (196) and adenoviral infection (197). During endotoxin-mediated lung injury STAT-3 regulates surfactant phospholipid synthesis and secretion (198). These studies in acute lung injury reveal a role of STAT-3 in type II AECs mainly in maintenance of surfactant homeostasis (199). Another analysis performing a genome wide mRNA analysis revealed that specific deletion of STAT-3 in type II 
AECs resulted in altered gene expression involved in cell growth, apoptosis, and lipid metabolism (200). These studies demonstrate that STAT-3 provides a protective effect in respiratory epithelial cells during acute lung injury. However, the role of STAT-3 in the setting of chronic inflammation and remodeling has not been addressed. My findings indicate that genetic removal and neutralization of IL-6 results in attenuated fibrosis in association with decreased STAT-3 activation in type II AECs. This suggests that the mechanisms by which IL-6 promotes fibrosis might involve STAT-3 activation in type II AECs.

One possible mechanism by which type II AECs might be involved in fibrosis is through the process known as EMT. Although the role of IL-6 on type II AECs with regards to EMT remains to be investigated, the potential of IL-6 to induce EMT in other cell types have been confirmed. In human breast cancer cells, IL-6 is able to induce EMT phenotype by activating STAT-3 and upregulating the expression of vimentin, N-cadherin, Snail, and Twist, in conjunction with impaired expression of E-cadherin (193). Other reports demonstrate that STAT-3 trans-activates Twist gene expression (201), which is a transcription factor involved in the activation of EMT. In addition, in ovarian carcinomas, crosstalk between EGF and IL-6R $\alpha$ mediates EMT by increasing Ncadherin and vimentin expression in a STAT-3 dependent manner (202). Other reports have studied the role of IL-6 in mesenchymal cells, human airway smooth muscle cells were reported not to express the mIL-6R $\alpha$ and exposure to IL-6 trans-signaling resulted in an increased expression of eotaxin and VEGF (203). IL-6 trans-signaling on human airway epithelial cells in response to bacteria 
pathogens lead to activation of MCP-1 and inhibition of IL-8 in the same cells (204). Hence, bacterial stimulation on airway epithelial cells induces IL-6 expression and activates IL-6R $\alpha$ shedding by TACE subsequently promoting autocrine-mediated IL-6 trans-signaling events. Similar results involving exposure of azoxymethane to mice leads to colonic epithelial cells inducing sIL$6 \mathrm{R} \alpha$ biosynthesis and STAT-3 activation, which in turns accelerates colon carcinogenesis (205). Other studies confirm that mice exposed to dextran sodium sulfate leads to colon carcinogenesis but through the induction of the IL6/sIL-6R $\alpha$ complex by laminar propia macrophages on colonic epithelial cells (206). This effect was associated with increased gp130 and decreased IL-6R $\alpha$ expression on colonic epithelial cells, suggesting a synchronized upregulation of IL-6 trans-signaling and reduction of classical IL-6 signaling. Accordingly, it has been established that classical signaling is associated with anti-inflammatory properties while trans-signaling with chronic and pro-inflammatory effects. Hence, it is conceivable that IL-6 might play diverse roles on epithelial cells, displaying protective effects via classical signaling and perhaps chronic and remodeling properties by means of trans-signaling activation. Our findings suggest a fibrotic contribution of IL-6 in type II AECs via the STAT-3 pathway in both models analyzed. Induction of EMT was observed on different alveolar epithelial cell lines with exposure to IL-6 in combination with the sIL-6R $\alpha$. The IL6/sIL-6R $\alpha$ complex was able to enhance EMT by increasing expression of vimentin and $\alpha$-SMA in these cells. Furthermore, induction of EMT by the IL6/sIL-6R $\alpha$ complex was shown to be associated with enhanced activation of the 
transcription factors STAT-3 and TWIST, which is a master regulator of EMT. TWIST is a known transcription factor involved in the regulation of metastasis, a multistep process where primary tumors spread to establish secondary tumors in distant sites (195). TWIST is a master regulator of embryonic morphogenesis and contributes to tumor metastasis by promoting EMT (195). It has been shown that TWIST is able to induce loss of E-cadherin-mediated cell-cell adhesion, promote cell motility, and induce mesenchymal markers (195). Hence, activation of TWIST via STAT-3 establishes a link between IL-6 and EMT. Thus, this IL6/sIL-6R $\alpha$ complex provides an alternative pathway by which IL-6 could potentially mediate the fibrotic development caused by increased adenosine concentrations.

Several studies have reported elevations of IL-6 in patients of inflammatory and fibrotic lung diseases such as asthma, COPD, sarcoidosis, cystic fibrosis, and IPF $(113,114,121)$. For instance, bronchial epithelial cells and mast cells from asthmatics produce IL-6 $(117,207)$, and alveolar macrophages from asthmatics secrete IL-6 upon allergen exposure (115). In addition, BALF and sputum from symptomatic asthmatics have increased IL-6 levels compared to asymptomatic asthmatics (118). These findings suggest that IL-6 is involved in the pathogenesis of many lung diseases. To further corroborate our findings and the importance of IL-6 signaling from COPD and IPF patients, we analyzed the expression of IL-6 and phospho-STAT-3 in lung tissue obtained from the Lung Tissue Research Consortium (LTRC). Patients with preserved lung function were used as control group in comparison to stage 4 
COPD and severe IPF patients. In COPD patients, increased IL-6 expression was observed mainly on macrophages whereas increased STAT-3 activation was detected in alveolar epithelial cells. This suggests a paracrine loop between inflammatory cells producing IL-6 and AECs being the cellular targets thereby augmenting disease conditions. In severe IPF patients, both IL-6 and phophoSTAT-3 expression were localized in hyperplastic epithelial cells. This suggests an autocrine IL-6 signaling where remodeled airway epithelial cells are both secreting and being the target of IL-6. This autocrine feedback loop represents a plausible mechanism by which IL-6 signaling exacerbates fibrotic pathologies by contributing to the differentiation of hyperplastic epithelial cells and increasing fibroblast foci accumulation, which are the site of active disease. In addition, it has been shown that following the engagement of the $A_{2 B} R$ on alveolar macrophages isolated from COPD and IPF patients produce increased IL-6 transcript and protein levels (208). Furthermore, elevations of the sIL-6R $\alpha$ were only detected at the affected lobe from the patient of IPF. The same patient did not demonstrated increased elevations of the sIL-6R $\alpha$ from the unaffected lobe. This clearly indicates that the production of the sIL-6R $\alpha$ is only generated in the setting of chronic injury. Thus, the slL-6R $\alpha$ levels are a clear indication of disease severity. These results substantiate the findings observed in our mouse models further demonstrating the relevance and importance of IL-6 signaling in human lung diseases. These studies have important implications for the use of adenosine and/or IL-6 blocking reagents in the treatment of pulmonary disorders where fibrosis is a detrimental component. 


\section{CHAPTER SIX}

\section{CONCLUDING REMARKS AND}

FUTURE DIRECTIONS 


\section{CONCLUSION}

Substantial information and studies demonstrate that adenosine elevations regulate pathways that promote the pathogenesis of chronic lung diseases. Studies in the $A d a^{-/-}$mice, an adenosine-mediated pulmonary injury model, reveal that adenosine is sufficient to regulate aspects of pulmonary phenotypes; however, the mechanism by which adenosine is able to regulate the chronic state of these diseases via the mediation of different factors remains unknown. My overall findings suggest that increased elevations of IL-6 are associated with increased STAT-3 activation in conjunction with increased pulmonary inflammation, alveolar destruction, and fibrosis (Fig 6.1). In this dissertation, experiments in Chapter Three demonstrate that macrophages and bronchial epithelial cells are the source of adenosine-driven IL-6 elevation, whereas alveolar epithelial cells are target cells of IL-6 via STAT-3 activation. In addition, genetic removal and neutralization of IL-6 in $A d a^{-/-}$mice is associated with diminished airway remodeling, attenuated lung inflammation, and reduced fibrosis in conjunction with decreased STAT-3 activation. Furthermore, experiments in Chapter Four utilized the bleomycin model, which is the most common model of pulmonary fibrosis to assess the therapeutic potential of antifibrotic agents. The genetic removal and neutralization of IL-6 in the bleomycin model is associated with decreased lung inflammation and attenuated fibrosis in conjunction with diminished STAT-3 activation. Therefore, our findings from Chapter Three and Four and published results in the literature suggest an 
essential role of IL-6 in the pathogenesis of inflammatory and fibrotic lung diseases.

These chapters indicate that targeting IL-6 can provide potential therapeutic benefits for chronic inflammatory and fibrotic diseases. Moreover, experiments in Chapter Five focus on determining the mechanism of action by which IL-6 induces fibrosis (Fig 6.1). IL-6 trans-signaling is elevated in Ada ${ }^{-/}$ mice and the bleomycin model. Also, IL-6 trans-signaling enhances EMT in vitro. This enhancement of EMT is characterized by increased expression of vimentin and $\alpha$-SMA in association with increased activation of STAT-3 and TWIST, a known master regulator of EMT. To the best of our knowledge, these experiments are the first to show IL-6 trans-signaling has direct fibrotic properties on alveolar epithelial cells. Also in Chapter Five, our findings in our models were corroborated in the lungs of IPF patients. Also, sIL-6R $\alpha$ levels were elevated in BALF from the affected lobe of an IPF patient together with increased IL-6 and STAT-3 activation. Thus, these studies demonstrate that IL-6 contributes to the inflammatory and fibrotic processes involved in chronic lung diseases. Thus, our working model is that elevated adenosine engages the $A_{2 B} R$ to increase IL-6 production from macrophages. In turn, IL-6 activates STAT-3 in target cells, mainly alveolar epithelial cells, to contribute to the pathology associated with these models via trans-signaling. Based on this research, treatment with the IL-6 neutralizing antibodies leads to the functional halting of STAT-3 activation in alveolar epithelial cells, which consequently inhibits the transcriptional activation of the pathways involved in the development of the chronic pathological 
symptoms observed in these models. Thus, IL-6 is involved in the development of pathological features associated with adenosine-mediated pulmonary injury and bleomycin-induced pulmonary fibrosis. These observations indicate that IL-6 signaling represents a potential therapeutic target for chronic lung diseases.

\section{FUTURE DIRECTIONS}

\section{DETERMINATION OF THE SOURCE OF SOLUBLE IL-6 RECEPTOR}

This dissertation demonstrated that IL-6 trans-signaling is functional in the setting of pathological conditions in both $\mathrm{Ada}^{-/-}$mice and the bleomycin model. Yet, one question remains unanswered, namely what is the source of the sIL6ra? To determine the cells that produce the proteolytic cleavage slL-6R (PCslL-6R), an experiment will be performed where isolated macrophages from both models will be analyzed using immunostaining for the expression of the mIL-6R $\alpha$. After confirming that these macrophages express the $\mathrm{mIL}-6 \mathrm{R} \alpha$, they will be incubated with the known protease ADAM17. This incubation is expected to generate the PC-sIL-6R. In addition, an ADAM17 inhibitor will be introduced to test if the PC-sIL-6R production is prevented. Measurements of ADAM17 with an ELISA assay in the plasma and BALF will further demonstrate the local production responsible for the increased sIL-6R levels. 


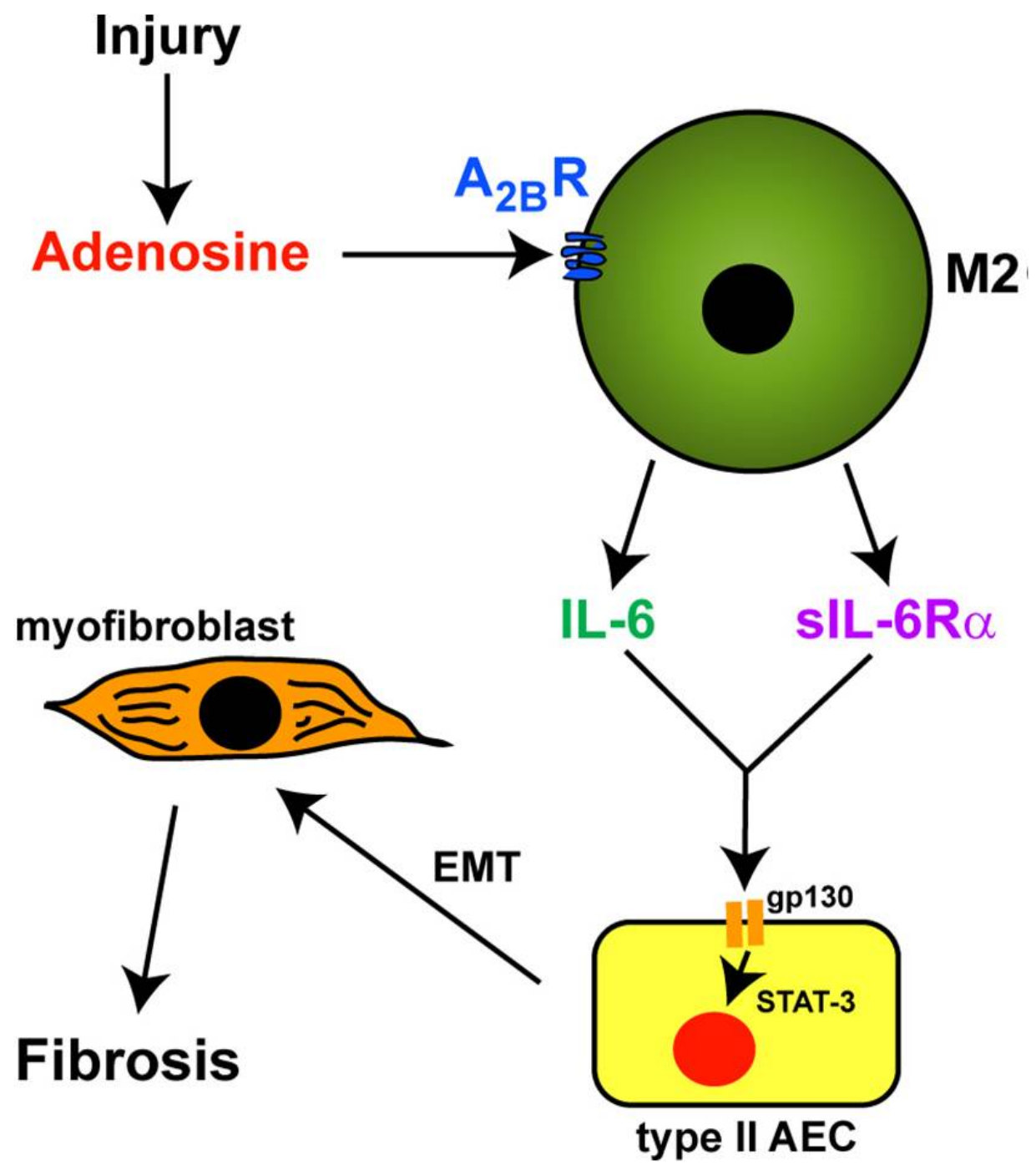

Figure 6.1

\section{Working Model}

After tissue injury, elevated adenosine levels engage the $A_{2 B} R$ on activated macrophages (M2) leading to increased IL-6 production and IL-6R $\alpha$ shedding. IL-6 trans-signaling involves the binding of the IL-6/sIL-6R $\alpha$ complex to gp130 receptors on type II alveolar epithelial cells (AECs) resulting in the phosphorylation and nuclear translocation of STAT-3. Activation of STAT-3 on these cells acts as a transcription factor in target genes that enhance the process of epithelial-to-mesenchymal transition (EMT), which is known to contribute to fibrosis via the accumulation of myofibroblasts and collagen production. 
Our results demonstrated that measurements of the sIL-6R $\alpha$ in the BALF and plasma from both models indicated that the sIL-6R $\alpha$ is produced locally rather than systemically. This is inferred since only BALF and not plasma display increased sIL-6R $\alpha$ levels. However, in this study no confirmation was made as to which slL-6R $\alpha$ isoform was increased during disease conditions of both models. Thus, differentiation of the two sIL-6R $\alpha$ isoforms needs to be done in order indicate the predominance of the alternative spliced sIL-6R $\alpha$ (AS-sIL-6R $\alpha$ ) or the proteolytic cleavage slL-6R $\alpha$ (PC-sIL-6R $\alpha$ ). To confirm the generation mechanism of the sIL-6R $\alpha$, an ELISA against the recognition sequence of the AS-sIL-6R $\alpha$ will be used from both plasma and BALF. Detecting the levels of the AS-sIL-6R $\alpha$ from both the plasma and BALF will demonstrate if the increased slL-6R $\alpha$ levels are produced locally by proteolytic cleavage or systemically by the alternative splice mechanism.

In addition, our results from BALF obtained from human patients of IPF demonstrated that sIL-6R $\alpha$ levels are elevated in the affected lobes of the individual whereas unaffected lobes display decreased levels of this receptor. This suggests that the sIL-6R $\alpha$ is generated only at the site of injury and increased levels of this receptor correlate with disease severity. However, our results are derived only from one patient due to the difficulty in obtaining human samples for basic research. Thus, future directions involved to further increase the number of human patients analyzed to corroborate these findings. 


\section{CHARACTERIZATION OF THE EFFECT OF STAT-3 INHIBITION ON IL-6- INDUCED FIBROSIS}

Observations in this dissertation demonstrated that IL-6-dependent increased phospho-STAT-3 activation is associated with pulmonary pathology. However, at this stage it is difficult to determine cause and effect between phospho-STAT-3 and pulmonary pathology or fibrosis. To assess the contribution of IL-6 via the STAT-3 pathway to the fibrotic response, type II AECs in the presence of IL-6 and the sIL-6R $\alpha$ will be treated with an STAT-3 siRNA. As a control, a scramble siRNA will be used to treat these cells. Treatment of IL6-activated type II AECs with the STAT-3 siRNA will determine which of the IL-6 activities related to fibrosis are activated by the STAT-3 pathway. If IL-6 induces EMT on type II AECs via the STAT-3 pathway, then treatment with the STAT-3 siRNA will be able to preserve the ATII phenotype and prevent the excessive ECM deposition common to fibrogenesis.

Treatment with the selective STAT-3 inhibitor CPA-7 can be used as a confirmatory assay to determine the effect of IL-6 via the STAT-3 or as an alternative approach in case the siRNA targeted approach does not completely silence the STAT-3 gene. An additional alternative approach will consists of obtaining primary type II AECs from conditional STAT-3 knockout mice. Treating these type II AECs, which do not express STAT-3, will not develop the EMT effects presumably induced by IL-6. In case that the MAPK or PI3K pathway is involved for fibrotic activities, the same approach will be used, namely the siRNA target approach and pharmacological inhibitors for ERK and Akt. 
Assessment of the pathway by which IL-6 contributes to features of pulmonary fibrosis is limited to the STAT-3 pathway. Although IL-6 activation is thought to be mainly STAT-3 mediated, other downstream signaling pathways are activated besides the STAT-3 such as the Ras/Raf/Mitogen-Activated Protein Kinases (MAPKs)/ERK and the phosphatidylinositol 3-kinase (PI3K)/Akt. These pathways are activated by IL-6 and elicit a variety of responses such as myeloma cell growth, survival, and drug resistance via the MAP Kinase pathway (209) and protection from TGF- $\beta$-induced apoptosis on hepatoma cells via the PI3K pathway (210). Therefore, an alternative approach to assess the mechanism involved in IL-6-induced fibrosis will include measuring the activity of the MAPK/ERK and the PI3K/Akt pathways. Western blot analysis measuring ERK and Akt phosphorylated proteins will determine the activity of these pathways when type II AECs are treated with IL-6 and sIL-6R together.

\section{DETERMINATION OF EPITHELIAL-TO-MESENCHYMAL TRANSITION IN THE ADA-DEFICIENT MICE AND THE BLEOMYCIN MODEL.}

Although on the previous chapter it was demonstrated in vitro that IL-6 trans-signaling is able to enhance EMT in alveolar epithelial cell lines, no direct evidence was provided that this process is occurring in vivo. To move from in vitro experiments to an in vivo setting, lung sections from $A d a^{-/-}$mice and bleomycin-exposed mice will be triple stained for E-cadherin, phospho-STAT-3, and $\alpha$-SMA. This triple staining will allow identifying type II AECs that are transitioning in losing their epithelial-like phenotype (E-cadherin) and becoming mesenchymal in nature ( $\alpha$-SMA). The phospho-STAT-3 staining will identify the 
cells that are undergoing EMT due to STAT-3 activation. According to our results, $\mathrm{Ada}^{-/-}$mice and bleomycin-exposed mice subjected to genetic removal or neutralization of should demonstrate reduced expression of this transitioning phase. Thus, future studies will determine the in vivo role of IL-6 in inducing EMT on type II AECs, which is the remodeling effect seen in pulmonary fibrosis $(158,166)$.

\section{IL-6 AS A THERAPEUTIC TARGET FOR CHRONIC LUNG DISEASE}

The results obtained from this dissertation indicate that targeting IL-6 could be beneficial in patients with chronic lung disease. In particular, it was demonstrated that pulmonary fibrosis is attenuated by blocking IL-6 in mice. Tocilizumab or Actemra is a humanized monoclonal antibody (mAb) targeting the IL-6 receptor (91), which inhibits both IL-6 classical and trans-signaling. The development of this humanized anti-IL-6 receptor mAb was pioneered for the treatment of the rare Castleman's disease and later was approved to treat rheumatoid arthritis (RA) (211). Significant improvement was observed in RA patients treated with Actemra compared to standard treatments alone. However, IL-6 blockers have not been considered for the treatment of chronic lung diseases. Considerations in using a humanized mAb against IL-6 must include the potential adverse effects of blocking such a pleiotropic cytokine. The main side-effect in blocking IL-6 could be the inability of the immune system to fight infections caused by bacteria, fungi, or viruses. It has been reported that RA patients while taking Actemra have died from these infections, such as 
tuberculosis and Epstein-Barr virus (211). In addition, other side-effects include gastrointestinal perforation, neutropenia, thrombocytopenia, increased liver enzymes, and elevated lipid parameters (212). Actemra is a geneticallyengineered $\mathrm{mAb}$ that is humanized from a mouse antihuman IL-6 receptor using the complementarity determining regions (CDRs), which are the most variable part of the antibody and determine its affinity and specificity for specific antigens. The humanized anti-human IL-6 receptor antibody has resulted in reduced antigenicity in humans. Thus, the advantage of using humanized Actemra is prolongation of its half-life and rarely causes production of neutralizing antibodies against the drug. Inhibition of IL-6 may also aid this feature since IL-6 induces antibody production (211).

IL-6 is a major player in driving the immune system with the potential to have pro- or anti-inflammatory properties. IL-6 remains at low levels in healthy individuals but increases dramatically during an immune response targeting different immune cells, such as monocytes, macrophages, dendritic dells, B cells and T cells. In response to injury, infection, or other factors, IL-6 induces an acute phase reaction marked by an increase in acute-phase proteins such as Creactive protein and fibrinogen. In addition, IL-6 not only favors the shift to a Th2 over Th1 response; but in combination with TGF- $\beta$, IL-6 is able to inhibit Treg cells and induce Th17 differentiation marked by increased IL-17 production. This Th17 subset of $T$ cells is involved in autoimmune diseases. To add to this complexity, IL-6 is able to activate cells that lack the mIL-6R $\alpha$ by forming the IL6/sIL-6R $\alpha$ complex and binding to gp130, a process known as trans-signaling. 
Hence, this complexity provides three different ways to inhibit IL-6 signaling by blocking the cytokine, the receptor, or the IL-6/sIL-6-R $\alpha$ complex. Blocking IL-6 signaling with humanized $\mathrm{mAb}$ against the receptor is associated with a degree of abnormalities. Targeting IL-6 and not the receptor presumably is associated with reduced side effects, especially in the liver since hepatocytes highly express the $\mathrm{mIL}-6 \mathrm{R} \alpha$. This makes IL-6 a decidedly more attractive therapeutic target. The third option is to block just IL-6 trans-signaling. This can be done by trapping the IL-6/sIL-6R $\alpha$ complex with the soluble gp130 fusion protein thereby preventing the activation of target cells. This resembles more a natural mechanism by allowing classical IL-6 signaling to be functional and most probably with an improved side-effect profile. The most common side-effect in IL-6 and IL-6 receptor blocking antibodies is the susceptibility to opportunistic infections since it weakens the body's immune defense. However, blocking IL-6 trans-signaling allows classical signaling to be activated as needed. Nevertheless, there is a need for clinical trials to determine which of these inhibitors compared to one another would be better for a particular disease.

Regarding inhibition of IL-6 signaling in patients with Chronic Lung Disease, therapeutic trials need to be performed to determine the efficacy and safety of these different blockers of IL-6. The different approaches to block IL-6 signaling are to inhibit IL-6 production $\left(A_{2 B} R\right.$ antagonism), direct neutralization of IL-6, blockage to the IL-6 receptor, and abduction of the IL-6/sIL-6R- $\alpha$. Another possibility is to inhibit the intracytoplasmic gp130 signal, thereby inhibiting STAT3 activation. Concerning the role of IL-6 in pulmonary fibrosis, treatment of IPF 
patients with the different options of blocking IL-6 signaling would determine which approach is more beneficial by monitoring the outcome and assessing the side-effects. Hence, long-term observational studies using these approaches in patients with IPF are needed to determine the efficacy of blocking IL-6 in chronic lung disease.

\section{SUMMARY}

This dissertation focuses on the confirmation of the contribution of IL-6 to features of inflammatory and fibrotic lung diseases in both the $A d a^{--}$mice and bleomycin-exposed mice in order to provide a better understanding of the development of these diseases. Collectively, the neutralization of IL-6 and the genetic removal of IL-6 in both models provided a comprehensive analysis regarding the contribution of IL-6 to disease progression in these models. Hence, the IL-6 signaling mechanism represents a potential therapeutic application for these deadly disorders. 


\section{References}

1. Vestbo, J., and Prescott, E. 1998. Update on the "Dutch hypothesis" for chronic respiratory disease. Thorax. 53:S15-19.

2. Thannickal, V.J., Toews, G.B., White, E.S., Lynch, J.P., 3rd, and Martinez, F.J. 2004. Mechanisms of pulmonary fibrosis. Annu Rev Med 55:395-417.

3. Sime, P.J., and O'Reilly, K.M. 2001. Fibrosis of the lung and other tissues: new concepts in pathogenesis and treatment. Clin Immunol 99:308-319.

4. Kung, H.C., Hoyert, D.L., Xu, J., and Murphy, S.L. 2008. Deaths: final data for 2005. Natl Vital Stat Rep. 56:1-120.

5. Elias, J.A., Lee, C.G., Zheng, T., Ma, B., Homer, R.J., and Zhu, Z. 2003. New insights into the pathogenesis of asthma. J Clin Invest 111:291-297.

6. O'Byrne P, M., and Postma, D.S. 1999. The many faces of airway inflammation. Asthma and chronic obstructive pulmonary disease. Asthma Research Group. Am J Respir Crit Care Med. 159:S41-63.

7. Panos, R.J., Mortenson, R.L., Niccoli, S.A., and King, T.E., Jr. 1990. Clinical deterioration in patients with idiopathic pulmonary fibrosis: causes and assessment. Am J Med 88:396-404.

8. Tillie-Leblond, I., Pugin, J., Marquette, C.H., Lamblin, C., Saulnier, F., Brichet, A., Wallaert, B., Tonnel, A.B., and Gosset, P. 1999. Balance between proinflammatory cytokines and their inhibitors in bronchial lavage from patients with status asthmaticus. Am $J$ Respir Crit Care Med. 159:487-494. 
9. Mohsenin, A., and Blackburn, M.R. 2006. Adenosine signaling in asthma and chronic obstructive pulmonary disease. Curr Opin Pulm Med 12:5459.

10. Rabe, B., Chalaris, A., May, U., Waetzig, G.H., Seegert, D., Williams, A.S., Jones, S.A., Rose-John, S., and Scheller, J. 2008. Transgenic blockade of interleukin 6 transsignaling abrogates inflammation. Blood. 111:1021-1028. Epub 2007 Nov 1027.

11. Gharaee-Kermani, M., Hu, B., Thannickal, V.J., Phan, S.H., and Gyetko, M.R. 2007. Current and emerging drugs for idiopathic pulmonary fibrosis. Expert Opin Emerg Drugs. 12:627-646.

12. Collard, H.R., and King, T.E., Jr. 2001. Treatment of idiopathic pulmonary fibrosis: the rise and fall of corticosteroids. Am J Med. 110:326-328.

13. Lynch, J., and Toews, G. 1998. Idiopathic pulmonary fibrosis. In Pulmonary Diseases and Disorders. A.P. Fishman, J.A. Elias, J.A. Fishman, M.A. Grippi, L.R. Kaiser, and R.M. Senior, editors. New york: McGraw-Hill. 1069-1084.

14. Bernstein, D.M., and Hoskins, J.A. 2006. The health effects of chrysotile: current perspective based upon recent data. Regul Toxicol Pharmacol. 45:252-264. Epub 2006 Jul 2011.

15. Wagner, J.A., and Gardner, P. 1997. Toward cystic fibrosis gene therapy. Annu Rev Med. 48:203-216. 
16. Caminati, A., and Harari, S. 2006. Smoking-related interstitial pneumonias and pulmonary Langerhans cell histiocytosis. Proc Am Thorac Soc. 3:299306.

17. Abid, S.H., Malhotra, V., and Perry, M.C. 2001. Radiation-induced and chemotherapy-induced pulmonary injury. Curr Opin Oncol. 13:242-248.

18. Majumdar, S., Li, D., Ansari, T., Pantelidis, P., Black, C.M., Gizycki, M., du Bois, R.M., and Jeffery, P.K. 1999. Different cytokine profiles in cryptogenic fibrosing alveolitis and fibrosing alveolitis associated with systemic sclerosis: a quantitative study of open lung biopsies. Eur Respir J. 14:251-257.

19. Steen, V.D., Conte, C., Owens, G.R., and Medsger, T.A., Jr. 1994. Severe restrictive lung disease in systemic sclerosis. Arthritis Rheum. 37:12831289.

20. Moller, D.R. 2003. Pulmonary fibrosis of sarcoidosis. New approaches, old ideas. Am J Respir Cell Mol Biol. 29:S37-41.

21. Lindell, K.O., and Jacobs, S.S. 2003. Idiopathic pulmonary fibrosis. Am J Nurs. 103:32-42; quiz 43.

22. Datta, A., Scotton, C.J., and Chambers, R.C. 1111. Novel Therapeutic Approaches for Pulmonary Fibrosis. Br J Pharmacol 2011:1476-5381.

23. Coultas, D.B., Zumwalt, R.E., Black, W.C., and Sobonya, R.E. 1994. The epidemiology of interstitial lung diseases. Am J Respir Crit Care Med. 150:967-972. 
24. Sheppard, D. 2001. Pulmonary fibrosis: a cellular overreaction or a failure of communication? J Clin Invest. 107:1501-1502.

25. Chuang-Tsai, S., Sisson, T.H., Hattori, N., Tsai, C.G., Subbotina, N.M., Hanson, K.E., and Simon, R.H. 2003. Reduction in fibrotic tissue formation in mice genetically deficient in plasminogen activator inhibitor-1. Am J Pathol. 163:445-452.

26. Atkinson, J.J., and Senior, R.M. 2003. Matrix metalloproteinase-9 in lung remodeling. Am J Respir Cell Mol Biol. 28:12-24.

27. Corry, D.B., Kiss, A., Song, L.Z., Song, L., Xu, J., Lee, S.H., Werb, Z., and Kheradmand, F. 2004. Overlapping and independent contributions of MMP2 and MMP9 to lung allergic inflammatory cell egression through decreased CC chemokines. Faseb J. 18:995-997. Epub 2004 Apr 2001.

28. Zhong, H., Belardinelli, L., Maa, T., and Zeng, D. 2005. Synergy between A2B adenosine receptors and hypoxia in activating human lung fibroblasts. Am J Respir Cell Mol Biol 32:2-8.

29. Hashimoto, N., Jin, H., Liu, T., Chensue, S.W., and Phan, S.H. 2004. Bone marrow-derived progenitor cells in pulmonary fibrosis. J Clin Invest. 113:243-252.

30. Kim, K.K., Kugler, M.C., Wolters, P.J., Robillard, L., Galvez, M.G., Brumwell, A.N., Sheppard, D., and Chapman, H.A. 2006. Alveolar epithelial cell mesenchymal transition develops in vivo during pulmonary fibrosis and is regulated by the extracellular matrix. Proc Natl Acad Sci U S A. 103:13180-13185. Epub 12006 Aug 13121. 
31. Hashimoto, N., Phan, S.H., Imaizumi, K., Matsuo, M., Nakashima, H., Kawabe, T., Shimokata, K., and Hasegawa, Y. 2009. Endothelialmesenchymal transition in bleomycin-induced pulmonary fibrosis. Am $\mathrm{J}$ Respir Cell Mol Biol 43:161-172.

32. Farkas, L., Gauldie, J., Voelkel, N.F., and Kolb, M. Pulmonary Hypertension and Idiopathic Pulmonary Fibrosis - A Tale of Angiogenesis, Apoptosis and Growth Factors. Am J Respir Cell Mol Biol 2010:5.

33. Sime, P.J., Xing, Z., Graham, F.L., Csaky, K.G., and Gauldie, J. 1997. Adenovector-mediated gene transfer of active transforming growth factorbeta1 induces prolonged severe fibrosis in rat lung. J Clin Invest. 100:768776.

34. Bonniaud, P., Margetts, P.J., Ask, K., Flanders, K., Gauldie, J., and Kolb, M. 2005. TGF-beta and Smad3 signaling link inflammation to chronic fibrogenesis. J Immunol. 175:5390-5395.

35. Kasai, H., Allen, J.T., Mason, R.M., Kamimura, T., and Zhang, Z. 2005. TGF-beta1 induces human alveolar epithelial to mesenchymal cell transition (EMT). Respir Res. 6:56.

36. Willis, B.C., Liebler, J.M., Luby-Phelps, K., Nicholson, A.G., Crandall, E.D., du Bois, R.M., and Borok, Z. 2005. Induction of epithelialmesenchymal transition in alveolar epithelial cells by transforming growth factor-beta1: potential role in idiopathic pulmonary fibrosis. Am J Pathol. 166:1321-1332. 
37. Ask, K., Martin, G.E., Kolb, M., and Gauldie, J. 2006. Targeting genes for treatment in idiopathic pulmonary fibrosis: challenges and opportunities, promises and pitfalls. Proc Am Thorac Soc. 3:389-393.

38. Gauldie, J., Kolb, M., and Sime, P.J. 2002. A new direction in the pathogenesis of idiopathic pulmonary fibrosis? Respir Res. 3:1. Epub 2001 Sep 2026.

39. Lee, C.G., Cho, S.J., Kang, M.J., Chapoval, S.P., Lee, P.J., Noble, P.W., Yehualaeshet, T., Lu, B., Flavell, R.A., Milbrandt, J., et al. 2004. Early growth response gene 1-mediated apoptosis is essential for transforming growth factor beta1-induced pulmonary fibrosis. J Exp Med. 200:377-389.

40. Moeller, A., Ask, K., Warburton, D., Gauldie, J., and Kolb, M. 2008. The bleomycin animal model: a useful tool to investigate treatment options for idiopathic pulmonary fibrosis? Int J Biochem Cell Biol. 40:362-382. Epub 2007 Aug 2030.

41. Desai, A., Victor-Vega, C., Gadangi, S., Montesinos, M.C., Chu, C.C., and Cronstein, B.N. 2005. Adenosine A2A receptor stimulation increases angiogenesis by down-regulating production of the antiangiogenic matrix protein thrombospondin 1. Mol Pharmacol. 67:1406-1413. Epub 2005 Jan 1426.

42. Kaczmarek, E., Koziak, K., Sevigny, J., Siegel, J.B., Anrather, J., Beaudoin, A.R., Bach, F.H., and Robson, S.C. 1996. Identification and characterization of CD39/vascular ATP diphosphohydrolase. J Biol Chem. 271:33116-33122. 
43. Resta, R., Hooker, S.W., Hansen, K.R., Laurent, A.B., Park, J.L., Blackburn, M.R., Knudsen, T.B., and Thompson, L.F. 1993. Murine ecto5'-nucleotidase (CD73): cDNA cloning and tissue distribution. Gene. 133:171-177.

44. Sala-Newby, G.B., Skladanowski, A.C., and Newby, A.C. 1999. The mechanism of adenosine formation in cells. Cloning of cytosolic 5'nucleotidase-I. J Biol Chem. 274:17789-17793.

45. Hermes, M., von Hippel, S., Osswald, H., and Kloor, D. 2005. Sadenosylhomocysteine metabolism in different cell lines: effect of hypoxia and cell density. Cell Physiol Biochem. 15:233-244.

46. Baldwin, S.A., Beal, P.R., Yao, S.Y., King, A.E., Cass, C.E., and Young, J.D. 2004. The equilibrative nucleoside transporter family, SLC29. Pflugers Arch. 447:735-743. Epub 2003 Jun 2028.

47. Gray, J.H., Owen, R.P., and Giacomini, K.M. 2004. The concentrative nucleoside transporter family, SLC28. Pflugers Arch. 447:728-734. Epub 2003 Jul 2011.

48. Blackburn, M.R., and Kellems, R.E. 1996. Regulation and function of adenosine deaminase in mice. Prog Nucleic Acid Res Mol Biol. 55:195226.

49. Fredholm, B.B. 2007. Adenosine, an endogenous distress signal, modulates tissue damage and repair. Cell Death Differ. 14:1315-1323. Epub 2007 Mar 1330. 
50. Driver, A.G., Kukoly, C.A., Ali, S., and Mustafa, S.J. 1993. Adenosine in bronchoalveolar lavage fluid in asthma. Am Rev Respir Dis 148:91-97.

51. Huszar, E., Vass, G., Vizi, E., Csoma, Z., Barat, E., Molnar, V.G., Herjavecz, I., and Horvath, I. 2002. Adenosine in exhaled breath condensate in healthy volunteers and in patients with asthma. Eur. Respir. J. 20:1393-1398.

52. Jacobson, K.A., and Gao, Z.G. 2006. Adenosine receptors as therapeutic targets. Nat Rev Drug Discov. 5:247-264.

53. Linden, J., Thai, T., Figler, H., Jin, X., and Robeva, A.S. 1999. Characterization of human $\mathrm{A}(2 \mathrm{~B})$ adenosine receptors: radioligand binding, western blotting, and coupling to $G(q)$ in human embryonic kidney 293 cells and HMC-1 mast cells. Mol Pharmacol. 56:705-713.

54. Englert, M., Quitterer, U., and Klotz, K.N. 2002. Effector coupling of stably transfected human $\mathrm{A} 3$ adenosine receptors in $\mathrm{CHO}$ cells. Biochem Pharmacol. 64:61-65.

55. Nyce, J.W., and Metzger, W.J. 1997. DNA antisense therapy for asthma in an animal model [see comments] [published erratum appears in Nature 1997 Nov 27;390(6658):424]. Nature 385:721-725.

56. Blackburn, M.R. 2003. Too much of a good thing: adenosine overload in adenosine-deaminase-deficient mice. Trends Pharmacol Sci 24:66-70.

57. Zhong, H., Chunn, J.L., Volmer, J.B., Fozard, J.R., and Blackburn, M.R. 2001. Adenosine-mediated mast cell degranulation in adenosine deaminase- deficient mice. J Pharmacol Exp Ther 298:433-440. 
58. Walker, B.A., Jacobson, M.A., Knight, D.A., Salvatore, C.A., Weir, T., Zhou, D., and Bai, T.R. 1997. Adenosine A3 receptor expression and function in eosinophils. Am J Respir Cell Mol Biol 16:531-537.

59. Johnson, H.G., and McNee, M.L. 1985. Adenosine-induced secretion in the canine trachea: modification by methylxanthines and adenosine derivatives. Br J Pharmacol 86:63-67.

60. Blackburn, M.R., Datta, S.K., and Kellems, R.E. 1998. Adenosine deaminase-deficient mice generated using a two-stage genetic engineering strategy exhibit a combined immunodeficiency. J Biol Chem 273:5093-5100.

61. Blackburn, M.R., and Kellems, R.E. 2005. Adenosine deaminase deficiency: metabolic basis of immune deficiency and pulmonary inflammation. Adv Immunol. 86:1-41.

62. Blackburn, M.R., Volmer, J.B., Thrasher, J.L., Zhong, H., Crosby, J.R., Lee, J.J., and Kellems, R.E. 2000. Metabolic consequences of adenosine deaminase deficiency in mice are associated with defects in alveogenesis, pulmonary inflammation, and airway obstruction. J Exp Med 192:159-170.

63. Chunn, J.L., Molina, J.G., Mi, T., Xia, Y., Kellems, R.E., and Blackburn, M.R. 2005. Adenosine-dependent pulmonary fibrosis in adenosine deaminase-deficient mice. J Immunol 175:1937-1946.

64. Blackburn, M.R., Aldrich, M., Volmer, J.B., Chen, W., Zhong, H., Kelly, S., Hershfield, M.S., Datta, S.K., and Kellems, R.E. 2000. The use of enzyme therapy to regulate the metabolic and phenotypic consequences of 
adenosine deaminase deficiency in mice. Differential impact on pulmonary and immunologic abnormalities. J Biol Chem 275:32114-32121.

65. Blackburn, M.R., Chun, G.L., Young, H.W.J., Chunn, J.L., Banerjee, S.K., and Elias, J.A. 2003. Adenosine mediates IL-13-induced inflammation and remodeling in the lung: evidence for an IL-13-adenosine amplification pathway. J. Clin. Invest. 112:332-344.

66. Ma, B., Blackburn, M.R., Lee, C.G., Homer, R.J., Liu, W., Flavell, R.A., Boyden, L., Lifton, R.P., Sun, C.X., Young, H.W., et al. 2006. Adenosine metabolism and murine strain-specific IL-4-induced inflammation, emphysema, and fibrosis. J Clin Invest 116:1274-1283.

67. Zhou, Y., Schneider, D.J., and Blackburn, M.R. 2009. Adenosine signaling and the regulation of chronic lung disease. Pharmacol Ther 123:105-116.

68. Jeffery, P.K. 2000. Comparison of the structural and inflammatory features of COPD and asthma. Giles F. Filley Lecture. Chest. 117:251S-260S.

69. Jeffery, P.K. 1994. Comparative morphology of the airways in asthma and chronic obstructive pulmonary disease. Am J Respir Crit Care Med. 150:S6-13.

70. Cushley, M.J., Tattersfield, A.E., and Holgate, S.T. 1984. Adenosineinduced bronchoconstriction in asthma. Antagonism by inhaled theophylline. Am Rev Respir Dis. 129:380-384.

71. Barnes, P.J. 1997. Current therapies for asthma. Promise and limitations. Chest. 111:17S-26S. 
72. Fredholm, B.B., and Persson, C.G. 1982. Xanthine derivatives as adenosine receptor antagonists. Eur J Pharmacol. 81:673-676.

73. Sun, Y., Wu, F., Sun, F., and Huang, P. 2008. Adenosine promotes IL-6 release in airway epithelia. $J$ Immunol. 180:4173-4181.

74. Walia, B., Castaneda, F.E., Wang, L., Kolachala, V.L., Bajaj, R., Roman, J., Merlin, D., Gewirtz, A.T., and Sitaraman, S.V. 2004. Polarized fibronectin secretion induced by adenosine regulates bacterial-epithelial interaction in human intestinal epithelial cells. Biochem J. 382:589-596.

75. Rees, D.A., Lewis, B.M., Lewis, M.D., Francis, K., Scanlon, M.F., and Ham, J. 2003. Adenosine-induced IL-6 expression in pituitary folliculostellate cells is mediated via $A 2 b$ adenosine receptors coupled to PKC and p38 MAPK. Br J Pharmacol. 140:764-772. Epub 2003 Sep 2022.

76. Schwaninger, M., Neher, M., Viegas, E., Schneider, A., and Spranger, M. 1997. Stimulation of interleukin-6 secretion and gene transcription in primary astrocytes by adenosine. J Neurochem 69:1145-1150.

77. Wagner, D.R., Bontemps, F., and van den Berghe, G. 1994. The AMPadenosine cycle is active during normoxia and impaired in ATP depletion in isolated rabbit cardiomyocytes. Adv Exp Med Biol 370:323-326.

78. Ritchie, P.K., Spangelo, B.L., Krzymowski, D.K., Rossiter, T.B., Kurth, E., and Judd, A.M. 1997. Adenosine increases interleukin 6 release and decreases tumour necrosis factor release from rat adrenal zona glomerulosa cells, ovarian cells, anterior pituitary cells, and peritoneal macrophages. Cytokine 9:187-198. 
79. Sun, C.X., Zhong, H., Mohsenin, A., Morschl, E., Chunn, J.L., Molina, J.G., Belardinelli, L., Zeng, D., and Blackburn, M.R. 2006. Role of A2B receptor signaling in adenosine-dependent pulmonary inflammation and injury. J Clin Invest 116:1-10.

80. Fiebich, B.L., Biber, K., Gyufko, K., Berger, M., Bauer, J., and van Calker, D. 1996. Adenosine A2b receptors mediate an increase in interleukin (IL)6 mRNA and IL-6 protein synthesis in human astroglioma cells. $J$ Neurochem 66:1426-1431.

81. Sitaraman, S.V., Merlin, D., Wang, L., Wong, M., Gewirtz, A.T., Si-Tahar, M., and Madara, J.L. 2001. Neutrophil-epithelial crosstalk at the intestinal lumenal surface mediated by reciprocal secretion of adenosine and IL-6. $J$ Clin Invest. 107:861-869.

82. Zhong, H., Belardinelli, L., Maa, T., Feoktistov, I., Biaggioni, I., and Zeng, D. 2004. $\mathrm{A}(2 \mathrm{~B})$ adenosine receptors increase cytokine release by bronchial smooth muscle cells. Am J Respir Cell Mol Biol 30:118-125.

83. Angeli, V., Faveeuw, C., Delerive, P., Fontaine, J., Barriera, Y., Franchimont, N., Staels, B., Capron, M., and Trottein, F. 2001. Schistosoma mansoni induces the synthesis of IL-6 in pulmonary microvascular endothelial cells: role of IL-6 in the control of lung eosinophilia during infection. Eur J Immuno/ 31:2751-2761.

84. Heinrich, P.C., Castell, J.V., and Andus, T. 1990. Interleukin-6 and the acute phase response. Biochem J. 265:621-636. 
85. Fernandez-Botran, R., Chilton, P.M., and Ma, Y. 1996. Soluble cytokine receptors: their roles in immunoregulation, disease, and therapy. Adv Immunol. 63:269-336.

86. Neta, R., Vogel, S.N., Sipe, J.D., Wong, G.G., and Nordan, R.P. 1988. Comparison of in vivo effects of human recombinant IL 1 and human recombinant IL 6 in mice. Lymphokine Res. 7:403-412.

87. Mclntosh, J.K., Jablons, D.M., Mule, J.J., Nordan, R.P., Rudikoff, S., Lotze, M.T., and Rosenberg, S.A. 1989. In vivo induction of IL-6 by administration of exogenous cytokines and detection of de novo serum levels of IL-6 in tumor-bearing mice. J Immunol. 143:162-167.

88. Hirano, T., and Kishimoto, T. 1992. Molecular biology and immunology of interleukin-6. Res Immunol. 143:723-724.

89. Hirano, T., Akira, S., Taga, T., and Kishimoto, T. 1990. Biological and clinical aspects of interleukin 6. Immunol Today. 11:443-449.

90. Heinrich, P.C., Behrmann, I., Haan, S., Hermanns, H.M., Muller-Newen, G., and Schaper, F. 2003. Principles of interleukin (IL)-6-type cytokine signalling and its regulation. Biochem J. 374:1-20.

91. Melton, L., and Coombs, A. 2008. Actemra poised to launch IL-6 inhibitors. Nat Biotechnol. 26:957-959.

92. Jostock, T., Mullberg, J., Ozbek, S., Atreya, R., Blinn, G., Voltz, N., Fischer, M., Neurath, M.F., and Rose-John, S. 2001. Soluble gp130 is the natural inhibitor of soluble interleukin-6 receptor transsignaling responses. Eur J Biochem. 268:160-167. 
93. Diehl, S., and Rincon, M. 2002. The two faces of IL-6 on Th1/Th2 differentiation. Mol Immunol 39:531-536.

94. Kamimura, D., Ishihara, K., and Hirano, T. 2003. IL-6 signal transduction and its physiological roles: the signal orchestration model. Rev Physiol Biochem Pharmacol 149:1-38.

95. Murakami, M., Hibi, M., Nakagawa, N., Nakagawa, T., Yasukawa, K., Yamanishi, K., Taga, T., and Kishimoto, T. 1993. IL-6-induced homodimerization of gp130 and associated activation of a tyrosine kinase. Science 260:1808-1810.

96. Ramos, B.F., Zhang, Y., and Jakschik, B.A. 1994. Neutrophil elicitation in the reverse passive Arthus reaction. Complement-dependent and independent mast cell involvement. J Immunol 152:1380-1384.

97. Heinrich, P.C., Behrmann, I., Haan, S., Hermanns, H.M., Muller-Newen, G., and Schaper, F. 2003. Principles of interleukin (IL)-6-type cytokine signalling and its regulation. Biochem J 374:1-20.

98. Heinrich, P.C., Behrmann, I., Muller-Newen, G., Schaper, F., and Graeve, L. 1998. Interleukin-6-type cytokine signalling through the gp130/Jak/STAT pathway. Biochem J 334 ( Pt 2):297-314.

99. Leonard, W.J., and O'Shea, J.J. 1998. Jaks and STATs: biological implications. Annu Rev Immunol 16:293-322.

100. Markert, M.L., Hershfield, M.S., Wiginton, D.A., States, J.C., Ward, F.E., Bigner, S.H., Buckley, R.H., Kaufman, R.E., and Hutton, J.J. 1987. 
Identification of a deletion in the adenosine deaminase gene in a child with severe combined immunodeficiency. J Immunol 138:3203-3206.

101. Nishimoto, N., and Kishimoto, T. 2006. Interleukin 6: from bench to bedside. Nat Clin Pract Rheumatol. 2:619-626.

102. Yu, H., Pardoll, D., and Jove, R. 2009. STATs in cancer inflammation and immunity: a leading role for STAT3. Nat Rev Cancer. 9:798-809.

103. Honda, M., Yamamoto, S., Cheng, M., Yasukawa, K., Suzuki, H., Saito, T., Osugi, Y., Tokunaga, T., and Kishimoto, T. 1992. Human soluble IL-6 receptor: its detection and enhanced release by HIV infection. J Immunol. 148:2175-2180.

104. Muller-Newen, G., Kohne, C., Keul, R., Hemmann, U., Muller-Esterl, W., Wijdenes, J., Brakenhoff, J.P., Hart, M.H., and Heinrich, P.C. 1996. Purification and characterization of the soluble interleukin-6 receptor from human plasma and identification of an isoform generated through alternative splicing. Eur J Biochem. 236:837-842.

105. Lust, J.A., Donovan, K.A., Kline, M.P., Greipp, P.R., Kyle, R.A., and Maihle, N.J. 1992. Isolation of an mRNA encoding a soluble form of the human interleukin-6 receptor. Cytokine. 4:96-100.

106. Mullberg, J., Schooltink, H., Stoyan, T., Gunther, M., Graeve, L., Buse, G., Mackiewicz, A., Heinrich, P.C., and Rose-John, S. 1993. The soluble interleukin-6 receptor is generated by shedding. Eur J Immunol. 23:473480. 
107. Horiuchi, K., Kimura, T., Miyamoto, T., Takaishi, H., Okada, Y., Toyama, Y., and Blobel, C.P. 2007. Cutting edge: TNF-alpha-converting enzyme (TACE/ADAM17) inactivation in mouse myeloid cells prevents lethality from endotoxin shock. J Immunol. 179:2686-2689.

108. Chalaris, A., Adam, N., Sina, C., Rosenstiel, P., Lehmann-Koch, J., Schirmacher, P., Hartmann, D., Cichy, J., Gavrilova, O., Schreiber, S., et al. 1617. Critical role of the disintegrin metalloprotease ADAM17 for intestinal inflammation and regeneration in mice. J Exp Med 207:16171624.

109. Black, R.A., Rauch, C.T., Kozlosky, C.J., Peschon, J.J., Slack, J.L., Wolfson, M.F., Castner, B.J., Stocking, K.L., Reddy, P., Srinivasan, S., et al. 1997. A metalloproteinase disintegrin that releases tumour-necrosis factor-alpha from cells. Nature. 385:729-733.

110. Matthews, V., Schuster, B., Schutze, S., Bussmeyer, I., Ludwig, A., Hundhausen, C., Sadowski, T., Saftig, P., Hartmann, D., Kallen, K.J., et al. 2003. Cellular cholesterol depletion triggers shedding of the human interleukin-6 receptor by ADAM10 and ADAM17 (TACE). J Biol Chem. 278:38829-38839. Epub 32003 Jun 38827.

111. Chalaris, A., Rabe, B., Paliga, K., Lange, H., Laskay, T., Fielding, C.A., Jones, S.A., Rose-John, S., and Scheller, J. 2007. Apoptosis is a natural stimulus of IL6R shedding and contributes to the proinflammatory transsignaling function of neutrophils. Blood. 110:1748-1755. Epub 2007 Jun 1713. 
112. Rose-John, S., Scheller, J., Elson, G., and Jones, S.A. 2006. Interleukin-6 biology is coordinated by membrane-bound and soluble receptors: role in inflammation and cancer. J Leukoc Biol 80:227-236.

113. Doganci, A., Sauer, K., Karwot, R., and Finotto, S. 2005. Pathological role of IL-6 in the experimental allergic bronchial asthma in mice. Clin Rev Allergy Immunol. 28:257-270.

114. Eddahibi, S., Chaouat, A., Tu, L., Chouaid, C., Weitzenblum, E., Housset, B., Maitre, B., and Adnot, S. 2006. Interleukin-6 gene polymorphism confers susceptibility to pulmonary hypertension in chronic obstructive pulmonary disease. Proc Am Thorac Soc. 3:475-476.

115. Gosset, P., Tsicopoulos, A., Wallaert, B., Vannimenus, C., Joseph, M., Tonnel, A.B., and Capron, A. 1991. Increased secretion of tumor necrosis factor alpha and interleukin- 6 by alveolar macrophages consecutive to the development of the late asthmatic reaction. J Allergy Clin Immunol. 88:561-571.

116. Bradding, P., Roberts, J.A., Britten, K.M., Montefort, S., Djukanovic, R., Mueller, R., Heusser, C.H., Howarth, P.H., and Holgate, S.T. 1994. Interleukin-4, -5 , and -6 and tumor necrosis factor-alpha in normal and asthmatic airways: evidence for the human mast cell as a source of these cytokines. Am J Respir Cell Mol Biol. 10:471-480.

117. Marini, M., Vittori, E., Hollemborg, J., and Mattoli, S. 1992. Expression of the potent inflammatory cytokines, granulocyte-macrophage-colony- 
stimulating factor and interleukin-6 and interleukin-8, in bronchial epithelial cells of patients with asthma. J Allergy Clin Immunol. 89:1001-1009.

118. Konno, S., Gonokami, Y., Kurokawa, M., Kawazu, K., Asano, K., Okamoto, K., and Adachi, M. 1996. Cytokine concentrations in sputum of asthmatic patients. Int Arch Allergy Immunol. 109:73-78.

119. Chunn, J.L., Mohsenin, A., Young, H.W., Lee, C.G., Elias, J.A., Kellems, R.E., and Blackburn, M.R. 2006. Partially adenosine deaminase-deficient mice develop pulmonary fibrosis in association with adenosine elevations. Am J Physiol Lung Cell Mol Physiol. 290:L579-587. Epub 2005 Oct 2028.

120. Elias, J.A., Zhu, Z., Chupp, G., and Homer, R.J. 1999. Airway remodeling in asthma. J Clin Invest 104:1001-1006.

121. Takizawa, H., Satoh, M., Okazaki, H., Matsuzaki, G., Suzuki, N., Ishii, A., Suko, M., Okudaira, H., Morita, Y., and Ito, K. 1997. Increased IL-6 and IL8 in bronchoalveolar lavage fluids (BALF) from patients with sarcoidosis: correlation with the clinical parameters. Clin Exp Immunol. 107:175-181.

122. Knight, D.A., Ernst, M., Anderson, G.P., Moodley, Y.P., and Mutsaers, S.E. 2003. The role of gp130/IL-6 cytokines in the development of pulmonary fibrosis: critical determinants of disease susceptibility and progression? Pharmacol Ther 99:327-338.

123. Smith, R.E., Strieter, R.M., Phan, S.H., Lukacs, N., and Kunkel, S.L. 1998. TNF and IL-6 mediate MIP-1alpha expression in bleomycin-induced lung injury. J Leukoc Biol 64:528-536. 
124. Saito, F., Tasaka, S., Inoue, K., Miyamoto, K., Nakano, Y., Ogawa, Y., Yamada, W., Shiraishi, Y., Hasegawa, N., Fujishima, S., et al. 2008. Role of interleukin-6 in bleomycin-induced lung inflammatory changes in mice. Am J Respir Cell Mol Biol 38:566-571.

125. Hasegawa, M., Sato, S., Ihn, H., and Takehara, K. 1999. Enhanced production of interleukin-6 (IL-6), oncostatin M and soluble IL-6 receptor by cultured peripheral blood mononuclear cells from patients with systemic sclerosis. Rheumatology (Oxford) 38:612-617.

126. Moodley, Y.P., Scaffidi, A.K., Misso, N.L., Keerthisingam, C., McAnulty, R.J., Laurent, G.J., Mutsaers, S.E., Thompson, P.J., and Knight, D.A. 2003. Fibroblasts isolated from normal lungs and those with idiopathic pulmonary fibrosis differ in interleukin-6/gp130-mediated cell signaling and proliferation. Am J Pathol 163:345-354.

127. Young, H.W., Molina, J.G., Dimina, D., Zhong, H., Jacobson, M., Chan, L.N., Chan, T.S., Lee, J.J., and Blackburn, M.R. 2004. A3 adenosine receptor signaling contributes to airway inflammation and mucus production in adenosine deaminase-deficient mice. $J$ Immunol 173:13801389.

128. Baran, C.P., Opalek, J.M., McMaken, S., Newland, C.A., O'Brien, J.M., Jr., Hunter, M.G., Bringardner, B.D., Monick, M.M., Brigstock, D.R., Stromberg, P.C., et al. 2007. Important roles for macrophage colonystimulating factor, $\mathrm{CC}$ chemokine ligand 2, and mononuclear phagocytes 
in the pathogenesis of pulmonary fibrosis. Am J Respir Crit Care Med. 176:78-89. Epub 2007 Apr 2012.

129. Zhou, Y., Mohsenin, A., Morschl, E., Young, H.W., Molina, J.G., Ma, W., Sun, C.X., Martinez-Valdez, H., and Blackburn, M.R. 2009. Enhanced airway inflammation and remodeling in adenosine deaminase-deficient mice lacking the A2B adenosine receptor. J Immunol. 182:8037-8046.

130. Ashcroft, T., Simpson, J.M., and Timbrell, V. 1988. Simple method of estimating severity of pulmonary fibrosis on a numerical scale. J Clin Pathol. 41:467-470.

131. Siddiquee, K., Zhang, S., Guida, W.C., Blaskovich, M.A., Greedy, B., Lawrence, H.R., Yip, M.L., Jove, R., McLaughlin, M.M., Lawrence, N.J., et al. 2007. Selective chemical probe inhibitor of Stat3, identified through structure-based virtual screening, induces antitumor activity. Proc Natl Acad Sci U S A. 104:7391-7396. Epub 2007 Apr 7326.

132. Rabe, K.F., Hurd, S., Anzueto, A., Barnes, P.J., Buist, S.A., Calverley, P., Fukuchi, Y., Jenkins, C., Rodriguez-Roisin, R., van Weel, C., et al. 2007. Global strategy for the diagnosis, management, and prevention of chronic obstructive pulmonary disease: GOLD executive summary. Am J Respir Crit Care Med. 176:532-555. Epub 2007 May 2016.

133. Ritchie, P.K., Spangelo, B.L., Krzymowski, D.K., Rossiter, T.B., Kurth, E., and Judd, A.M. 1997. Adenosine increases interleukin 6 release and decreases tumour necrosis factor release from rat adrenal zona 
glomerulosa cells, ovarian cells, anterior pituitary cells, and peritoneal macrophages. Cytokine. 9:187-198.

134. Angeli, V., Faveeuw, C., Delerive, P., Fontaine, J., Barriera, Y., Franchimont, N., Staels, B., Capron, M., and Trottein, F. 2001. Schistosoma mansoni induces the synthesis of IL-6 in pulmonary microvascular endothelial cells: role of IL-6 in the control of lung eosinophilia during infection. Eur J Immunol. 31:2751-2761.

135. Zhong, H., Belardinelli, L., Maa, T., Feoktistov, I., Biaggioni, I., and Zeng, D. 2004. $\mathrm{A}(2 \mathrm{~B})$ adenosine receptors increase cytokine release by bronchial smooth muscle cells. Am J Respir Cell Mol Biol. 30:118-125. Epub 2003 Jul 2010.

136. Chunn, J.L., Molina, J.G., Mi, T., Xia, Y., Kellems, R.E., and Blackburn, M.R. 2005. Adenosine-dependent pulmonary fibrosis in adenosine deaminase-deficient mice. J Immunol. 175:1937-1946.

137. Eckle, T., Faigle, M., Grenz, A., Laucher, S., Thompson, L.F., and Eltzschig, H.K. 2008. A2B adenosine receptor dampens hypoxia-induced vascular leak. Blood 111:2024-2035.

138. Yang, R., Han, X., Uchiyama, T., Watkins, S.K., Yaguchi, A., Delude, R.L., and Fink, M.P. 2003. IL-6 is essential for development of gut barrier dysfunction after hemorrhagic shock and resuscitation in mice. Am $J$ Physiol Gastrointest Liver Physiol. 285:G621-629. Epub 2003 May 2028.

139. Fielding, C.A., McLoughlin, R.M., McLeod, L., Colmont, C.S., Najdovska, M., Grail, D., Ernst, M., Jones, S.A., Topley, N., and Jenkins, B.J. 2008. 
IL-6 regulates neutrophil trafficking during acute inflammation via STAT3. J Immunol. 181:2189-2195.

140. Schneider, D.J., Lindsay, J.C., Zhou, Y., Molina, J.G., and Blackburn, M.R. 2009. Adenosine and osteopontin contribute to the development of chronic obstructive pulmonary disease. Faseb $\mathrm{J}$.

141. Jones, S.A. 2005. Directing transition from innate to acquired immunity: defining a role for IL-6. J Immunol. 175:3463-3468.

142. Kaplanski, G., Marin, V., Montero-Julian, F., Mantovani, A., and Farnarier, C. 2003. IL-6: a regulator of the transition from neutrophil to monocyte recruitment during inflammation. Trends Immunol. 24:25-29.

143. Sica, A., and Bronte, V. 2007. Altered macrophage differentiation and immune dysfunction in tumor development. J Clin Invest. 117:1155-1166.

144. Mills, C.D., Kincaid, K., Alt, J.M., Heilman, M.J., and Hill, A.M. 2000. M1/M-2 macrophages and the Th1/Th2 paradigm. J Immunol. 164:61666173.

145. McLoughlin, R.M., Jenkins, B.J., Grail, D., Williams, A.S., Fielding, C.A., Parker, C.R., Ernst, M., Topley, N., and Jones, S.A. 2005. IL-6 transsignaling via STAT3 directs $\mathrm{T}$ cell infiltration in acute inflammation. Proc Natl Acad Sci U S A. 102:9589-9594. Epub 2005 Jun 9523.

146. Korn, T., Mitsdoerffer, M., Croxford, A.L., Awasthi, A., Dardalhon, V.A., Galileos, G., Vollmar, P., Stritesky, G.L., Kaplan, M.H., Waisman, A., et al. 2008. IL-6 controls Th17 immunity in vivo by inhibiting the conversion of 
conventional T cells into Foxp3+ regulatory T cells. Proc Natl Acad Sci U S A. 105:18460-18465. Epub 12008 Nov 18417.

147. Kimura, A., and Kishimoto, T. 1830. IL-6: regulator of Treg/Th17 balance. Eur J Immunol 40:1830-1835.

148. Kusano, K., Miyaura, C., Inada, M., Tamura, T., Ito, A., Nagase, H., Kamoi, K., and Suda, T. 1998. Regulation of matrix metalloproteinases (MMP-2, -3, -9, and -13) by interleukin-1 and interleukin-6 in mouse calvaria: association of MMP induction with bone resorption. Endocrinology. 139:1338-1345.

149. Mihara, M., Moriya, Y., Kishimoto, T., and Ohsugi, Y. 1995. Interleukin-6 (IL-6) induces the proliferation of synovial fibroblastic cells in the presence of soluble IL-6 receptor. Br J Rheumatol. 34:321-325.

150. Eckle, T., Faigle, M., Grenz, A., Laucher, S., Thompson, L.F., and Eltzschig, H.K. 2008. A2B adenosine receptor dampens hypoxia-induced vascular leak. Blood. 111:2024-2035. Epub 2007 Dec 2024.

151. Eltzschig, H.K., Ibla, J.C., Furuta, G.T., Leonard, M.O., Jacobson, K.A., Enjyoji, K., Robson, S.C., and Colgan, S.P. 2003. Coordinated adenine nucleotide phosphohydrolysis and nucleoside signaling in posthypoxic endothelium: role of ectonucleotidases and adenosine A2B receptors. $J$ Exp Med 198:783-796.

152. Rogers, D.F. 2004. Airway mucus hypersecretion in asthma: an undervalued pathology? Curr Opin Pharmacol. 4:241-250. 
153. Neveu, W.A., Allard, J.B., Dienz, O., Wargo, M.J., Ciliberto, G., Whittaker, L.A., and Rincon, M. 2009. IL-6 is required for airway mucus production induced by inhaled fungal allergens. J Immunol. 183:1732-1738. Epub 2009 Jul 1710.

154. Chen, Y., Thai, P., Zhao, Y.H., Ho, Y.S., DeSouza, M.M., and Wu, R. 2003. Stimulation of airway mucin gene expression by interleukin (IL)-17 through IL-6 paracrine/autocrine loop. J Biol Chem. 278:17036-17043. Epub 12003 Mar 17036.

155. Pinart, M., Serrano-Mollar, A., Llatjos, R., Rocco, P.R., and Romero, P.V. 2009. Single and repeated bleomycin intratracheal instillations lead to different biomechanical changes in lung tissue. Respir Physiol Neurobiol. 166:41-46. Epub 2009 Jan 2021.

156. Tapkire, R., Kathiresan, N., and Satheesan, B. 2009. Bleomycin induced urethral stricture in Hodgkin's disease. Indian J Urol. 25:398-400.

157. Strieter, R.M., and Mehrad, B. 2009. New mechanisms of pulmonary fibrosis. Chest. 136:1364-1370.

158. Willis, B.C., duBois, R.M., and Borok, Z. 2006. Epithelial origin of myofibroblasts during fibrosis in the lung. Proc Am Thorac Soc. 3:377-382.

159. Qiu, Z., Fujimura, M., Kurashima, K., Nakao, S., and Mukaida, N. 2004. Enhanced airway inflammation and decreased subepithelial fibrosis in interleukin 6-deficient mice following chronic exposure to aerosolized antigen. Clin Exp Allergy. 34:1321-1328. 
160. Estep, J.M., O'Reilly, L., Grant, G., Piper, J., Jonsson, J., Afendy, A., Chandhoke, V., and Younossi, Z.M. 2009. Hepatic stellate cell and myofibroblast-like cell gene expression in the explanted cirrhotic livers of patients undergoing liver transplantation. Dig Dis Sci 55:496-504.

161. Smith, R.E., Strieter, R.M., Zhang, K., Phan, S.H., Standiford, T.J., Lukacs, N.W., and Kunkel, S.L. 1995. A role for C-C chemokines in fibrotic lung disease. J Leukoc Biol. 57:782-787.

162. Melendez, G.C., McLarty, J.L., Levick, S.P., Du, Y., Janicki, J.S., and Brower, G.L. Interleukin 6 mediates myocardial fibrosis, concentric hypertrophy, and diastolic dysfunction in rats. Hypertension. 56:225-231. Epub 2010 Jul 2016.

163. Gallucci, R.M., Lee, E.G., and Tomasek, J.J. 2006. IL-6 modulates alphasmooth muscle actin expression in dermal fibroblasts from IL-6-deficient mice. J Invest Dermatol. 126:561-568.

164. Kanzler, S., Lohse, A.W., Keil, A., Henninger, J., Dienes, H.P., Schirmacher, P., Rose-John, S., zum Buschenfelde, K.H., and Blessing, M. 1999. TGF-beta1 in liver fibrosis: an inducible transgenic mouse model to study liver fibrogenesis. Am J Physiol. 276:G1059-1068.

165. Stramer, B.M., Mori, R., and Martin, P. 2007. The inflammation-fibrosis link? A Jekyll and Hyde role for blood cells during wound repair. J Invest Dermatol. 127:1009-1017.

166. Cannito, S., Novo, E., Valfre di Bonzo, L., Busletta, C., Colombatto, S., and Parola, M. 2009. Epithelial-Mesenchymal Transition: from Molecular 
Mechanisms, Redox Regulation to Implications in Human Health and Disease. Antioxid Redox Signal 10:10.

167. Danto, S.I., Shannon, J.M., Borok, Z., Zabski, S.M., and Crandall, E.D. 1995. Reversible transdifferentiation of alveolar epithelial cells. Am $J$ Respir Cell Mol Biol. 12:497-502.

168. Kalluri, R., and Neilson, E.G. 2003. Epithelial-mesenchymal transition and its implications for fibrosis. J Clin Invest. 112:1776-1784.

169. Fan, J.M., Ng, Y.Y., Hill, P.A., Nikolic-Paterson, D.J., Mu, W., Atkins, R.C., and Lan, H.Y. 1999. Transforming growth factor-beta regulates tubular epithelial-myofibroblast transdifferentiation in vitro. Kidney Int. 56:14551467.

170. Desmouliere, A. 1995. Factors influencing myofibroblast differentiation during wound healing and fibrosis. Cell Biol Int. 19:471-476.

171. Desmouliere, A., Geinoz, A., Gabbiani, F., and Gabbiani, G. 1993. Transforming growth factor-beta 1 induces alpha-smooth muscle actin expression in granulation tissue myofibroblasts and in quiescent and growing cultured fibroblasts. J Cell Biol. 122:103-111.

172. Savagner, P. 2001. Leaving the neighborhood: molecular mechanisms involved during epithelial-mesenchymal transition. Bioessays. 23:912-923.

173. Masszi, A., Di Ciano, C., Sirokmany, G., Arthur, W.T., Rotstein, O.D., Wang, J., McCulloch, C.A., Rosivall, L., Mucsi, I., and Kapus, A. 2003. Central role for Rho in TGF-beta1-induced alpha-smooth muscle actin 
expression during epithelial-mesenchymal transition. Am J Physiol Renal Physiol. 284:F911-924. Epub 2002 Dec 2027.

174. Zavadil, J., and Bottinger, E.P. 2005. TGF-beta and epithelial-tomesenchymal transitions. Oncogene. 24:5764-5774.

175. Grunert, S., Jechlinger, M., and Beug, H. 2003. Diverse cellular and molecular mechanisms contribute to epithelial plasticity and metastasis. Nat Rev Mol Cell Biol. 4:657-665.

176. Tomasek, J.J., Gabbiani, G., Hinz, B., Chaponnier, C., and Brown, R.A. 2002. Myofibroblasts and mechano-regulation of connective tissue remodelling. Nat Rev Mol Cell Biol. 3:349-363.

177. Eyden, B. 2001. The myofibroblast: an assessment of controversial issues and a definition useful in diagnosis and research. Ultrastruct Pathol. 25:39-50.

178. McAnulty, R.J. 2007. Fibroblasts and myofibroblasts: their source, function and role in disease. Int J Biochem Cell Biol. 39:666-671. Epub 2006 Nov 2023.

179. Ogawa, M., LaRue, A.C., and Drake, C.J. 2006. Hematopoietic origin of fibroblasts/myofibroblasts: Its pathophysiologic implications. Blood. 108:2893-2896. Epub 2006 Jul 2813.

180. Hinz, B. 2007. Formation and function of the myofibroblast during tissue repair. J Invest Dermatol. 127:526-537.

181. Phan, S.H. 2002. The myofibroblast in pulmonary fibrosis. Chest. 122:286S-289S. 
182. Adler, K.B., Low, R.B., Leslie, K.O., Mitchell, J., and Evans, J.N. 1989. Contractile cells in normal and fibrotic lung. Lab Invest. 60:473-485.

183. Desmouliere, A., Chaponnier, C., and Gabbiani, G. 2005. Tissue repair, contraction, and the myofibroblast. Wound Repair Regen. 13:7-12.

184. Starr, R., and Hilton, D.J. 1999. Negative regulation of the JAK/STAT pathway. Bioessays. 21:47-52.

185. Roth, M., Nauck, M., Tamm, M., Perruchoud, A.P., Ziesche, R., and Block, L.H. 1995. Intracellular interleukin 6 mediates platelet-derived growth factor-induced proliferation of nontransformed cells. Proc Natl Acad Sci U S A. 92:1312-1316.

186. Kawaguchi, Y., Hara, M., and Wright, T.M. 1999. Endogenous IL-1alpha from systemic sclerosis fibroblasts induces IL-6 and PDGF-A. J Clin Invest. 103:1253-1260.

187. Moodley, Y.P., Scaffidi, A.K., Misso, N.L., Keerthisingam, C., McAnulty, R.J., Laurent, G.J., Mutsaers, S.E., Thompson, P.J., and Knight, D.A. 2003. Fibroblasts isolated from normal lungs and those with idiopathic pulmonary fibrosis differ in interleukin-6/gp130-mediated cell signaling and proliferation. Am J Pathol. 163:345-354.

188. DiCosmo, B.F., Geba, G.P., Picarella, D., Elias, J.A., Rankin, J.A., Stripp, B.R., Whitsett, J.A., and Flavell, R.A. 1994. Airway epithelial cell expression of interleukin-6 in transgenic mice. Uncoupling of airway inflammation and bronchial hyperreactivity. J Clin Invest. 94:2028-2035. 
189. Yoshida, M., Sakuma, J., Hayashi, S., Abe, K., Saito, I., Harada, S., Sakatani, M., Yamamoto, S., Matsumoto, N., Kaneda, Y., et al. 1995. A histologically distinctive interstitial pneumonia induced by overexpression of the interleukin 6 , transforming growth factor beta 1 , or platelet-derived growth factor B gene. Proc Natl Acad Sci U S A. 92:9570-9574.

190. Karmiol, S., Remick, D.G., Kunkel, S.L., and Phan, S.H. 1993. Regulation of rat pulmonary endothelial cell interleukin- 6 production by bleomycin: effects of cellular fatty acid composition. Am J Respir Cell Mol Biol. 9:628636.

191. Micallef, M., Hosokawa, M., Togashi, Y., and Kobayashi, H. 1992. Rat macrophage activation after treatment with the bleomycin group of antitumour antibiotics in vivo. Cancer Immunol Immunother. 35:106-112.

192. Chavey, C., Bibeau, F., Gourgou-Bourgade, S., Burlinchon, S., Boissiere, F., Laune, D., Roques, S., and Lazennec, G. 2007. Oestrogen receptor negative breast cancers exhibit high cytokine content. Breast Cancer Res. 9:R15.

193. Sullivan, N.J., Sasser, A.K., Axel, A.E., Vesuna, F., Raman, V., Ramirez, N., Oberyszyn, T.M., and Hall, B.M. 2009. Interleukin-6 induces an epithelial-mesenchymal transition phenotype in human breast cancer cells. Oncogene. 28:2940-2947. Epub 2009 Jul 2946.

194. Pozharskaya, V., Torres-Gonzalez, E., Rojas, M., Gal, A., Amin, M., Dollard, S., Roman, J., Stecenko, A.A., and Mora, A.L. 2009. Twist: a 
regulator of epithelial-mesenchymal transition in lung fibrosis. PLOS One. 4:e7559.

195. Yang, J., Mani, S.A., Donaher, J.L., Ramaswamy, S., Itzykson, R.A., Come, C., Savagner, P., Gitelman, I., Richardson, A., and Weinberg, R.A. 2004. Twist, a master regulator of morphogenesis, plays an essential role in tumor metastasis. Cell. 117:927-939.

196. Hokuto, I., Ikegami, M., Yoshida, M., Takeda, K., Akira, S., Perl, A.K., Hull, W.M., Wert, S.E., and Whitsett, J.A. 2004. Stat-3 is required for pulmonary homeostasis during hyperoxia. J Clin Invest. 113:28-37.

197. Matsuzaki, Y., Xu, Y., Ikegami, M., Besnard, V., Park, K.S., Hull, W.M., Wert, S.E., and Whitsett, J.A. 2006. Stat3 is required for cytoprotection of the respiratory epithelium during adenoviral infection. J Immunol. 177:527537.

198. Ikegami, M., Falcone, A., and Whitsett, J.A. 2008. STAT-3 regulates surfactant phospholipid homeostasis in normal lung and during endotoxinmediated lung injury. J Appl Physiol. 104:1753-1760. Epub 2008 Mar 1727.

199. Matsuzaki, Y., Besnard, V., Clark, J.C., Xu, Y., Wert, S.E., Ikegami, M., and Whitsett, J.A. 2008. STAT3 regulates ABCA3 expression and influences lamellar body formation in alveolar type II cells. Am J Respir Cell Mol Biol. 38:551-558. Epub 2007 Dec 2020. 
200. Xu, Y., Ikegami, M., Wang, Y., Matsuzaki, Y., and Whitsett, J.A. 2007. Gene expression and biological processes influenced by deletion of Stat3 in pulmonary type II epithelial cells. BMC Genomics. 8:455.

201. Cheng, G.Z., Zhang, W.Z., Sun, M., Wang, Q., Coppola, D., Mansour, M., Xu, L.M., Costanzo, C., Cheng, J.Q., and Wang, L.H. 2008. Twist is transcriptionally induced by activation of STAT3 and mediates STAT3 oncogenic function. J Biol Chem. 283:14665-14673. Epub 12008 Mar 14619.

202. Colomiere, M., Ward, A.C., Riley, C., Trenerry, M.K., Cameron-Smith, D., Findlay, J., Ackland, L., and Ahmed, N. 2009. Cross talk of signals between EGFR and IL-6R through JAK2/STAT3 mediate epithelialmesenchymal transition in ovarian carcinomas. Br J Cancer. 100:134-144. Epub 2008 Dec 2016.

203. Ammit, A.J., Moir, L.M., Oliver, B.G., Hughes, J.M., Alkhouri, H., Ge, Q., Burgess, J.K., Black, J.L., and Roth, M. 2007. Effect of IL-6 trans-signaling on the pro-remodeling phenotype of airway smooth muscle. Am J Physiol Lung Cell Mol Physiol 292:L199-206.

204. Gomez, M.I., Sokol, S.H., Muir, A.B., Soong, G., Bastien, J., and Prince, A.S. 2005. Bacterial induction of TNF-alpha converting enzyme expression and IL-6 receptor alpha shedding regulates airway inflammatory signaling. J Immunol. 175:1930-1936.

205. Rose-John, S., Mitsuyama, K., Matsumoto, S., Thaiss, W.M., and Scheller, J. 2009. Interleukin-6 trans-signaling and colonic cancer 
associated with inflammatory bowel disease. Curr Pharm Des. 15:20952103.

206. Matsumoto, S., Hara, T., Mitsuyama, K., Yamamoto, M., Tsuruta, O., Sata, M., Scheller, J., Rose-John, S., Kado, S., and Takada, T. 1543. Essential roles of IL-6 trans-signaling in colonic epithelial cells, induced by the IL-6/soluble-IL-6 receptor derived from lamina propria macrophages, on the development of colitis-associated premalignant cancer in a murine model. J Immunol 184:1543-1551.

207. Bradding, P., Roberts, J.A., Britten, K.M., Montefort, S., Djukanovic, R., Mueller, R., Heusser, C.H., Howarth, P.H., and Holgate, S.T. 1994. Interleukin-4, -5, and -6 and tumor necrosis factor-alpha in normal and asthmatic airways: evidence for the human mast cell as a source of these cytokines. Am J Respir Cell Mol Biol 10:471-480.

208. Zhou, Y., Murthy, J.N., Zeng, D., Belardinelli, L., and Blackburn, M.R. Alterations in adenosine metabolism and signaling in patients with chronic obstructive pulmonary disease and idiopathic pulmonary fibrosis. PLoS One 5:e9224.

209. Lentzsch, S., Chatterjee, M., Gries, M., Bommert, K., Gollasch, H., Dorken, B., and Bargou, R.C. 2004. PI3-K/AKT/FKHR and MAPK signaling cascades are redundantly stimulated by a variety of cytokines and contribute independently to proliferation and survival of multiple myeloma cells. Leukemia. 18:1883-1890. 
210. Chen, R.H., Chang, M.C., Su, Y.H., Tsai, Y.T., and Kuo, M.L. 1999. Interleukin-6 inhibits transforming growth factor-beta-induced apoptosis through the phosphatidylinositol 3-kinase/Akt and signal transducers and activators of transcription 3 pathways. J Biol Chem. 274:23013-23019.

211. Nishimoto, N., and Kishimoto, T. 2008. Humanized antihuman IL-6 receptor antibody, tocilizumab. Handb Exp Pharmacol::151-160.

212. Patel, A.M., and Moreland, L.W. Interleukin-6 inhibition for treatment of rheumatoid arthritis: a review of tocilizumab therapy. Drug 4:263-278. 


\section{VITAE}

Name of author: Mesias Pedroza

Date and place of birth: October 16th, 1982, Chicago, IL

High School:

Class of $2002-$

Sam Houston High School, Houston, TX

Undergraduate:

B.S. in Biotechnology, Cum Laude

University of Houston - Downtown, 2007

Post-graduate:

University of Texas at Houston Medical School

Graduate School of Biomedical Sciences

(PhD Program) 